University of Louisville

ThinkIR: The University of Louisville's Institutional Repository

Electronic Theses and Dissertations

4-2009

\title{
Lung nodule modeling and detection for computerized image analysis of low dose CT imaging of the chest.
}

Amal A. Farag

University of Louisville

Follow this and additional works at: https://ir.library.louisville.edu/etd

\section{Recommended Citation}

Farag, Amal A., "Lung nodule modeling and detection for computerized image analysis of low dose CT imaging of the chest." (2009). Electronic Theses and Dissertations. Paper 424.

https://doi.org/10.18297/etd/424

This Master's Thesis is brought to you for free and open access by ThinkIR: The University of Louisville's Institutional Repository. It has been accepted for inclusion in Electronic Theses and Dissertations by an authorized administrator of ThinkIR: The University of Louisville's Institutional Repository. This title appears here courtesy of the author, who has retained all other copyrights. For more information, please contact thinkir@louisville.edu. 


\title{
LUNG NODULE MODELING AND DETECTION FOR COMPUTERIZED IMAGE
} ANALYSIS OF LOW DOSE CT IMAGING OF THE CHEST

\author{
By
}

Amal A. Farag

B.S.E.E., University of Louisville, 2008

\author{
A Thesis \\ Submitted to the Faculty of the \\ University of Louisville \\ Speed Scientific School \\ As Partial Fulfillment of the Requirements \\ For the Professional Degree
}

MASTER OF ENGINEERING

Department of Electrical and Computer Engineering

April, 2009 


\section{LUNG NODULE MODELING AND DETECTION FOR COMPUTERIZED IMAGE}

ANALYSIS OF LOW DOSE CT IMAGING OF THE CHEST

Submitted by:

Amal A. Farag

A Thesis Approved on

By the Following Reading and Examination Committee:

James Graham, Thesis Director

Aly A. Farag, Thesis Co-director

Renato LaRocca

Robert Falk

Mohammed Ahmed 


\section{ACKNOWLEDGMENTS}

I would like to thank my thesis advisors Dr. James Graham and Dr. Aly Farag for their support and guidance, and Dr. Robert Falk, Dr. Renato LaRocca and Dr. Mohamed Ahmed, for serving on the thesis committee and being a part of this special milestone. I am grateful for Dr. Ahmed as my teacher in a number of classes.

I would like to thank all of the CVIP Lab members for their support and friendship; special thanks to Dr. Asem Ali and Dr. Dongqing Chen for sharing their knowledge and never hesitating to provide guidance or clarity. Also, to Shireen Elhabian who has been a colleague and great friend; I wish her luck and success in all her future endeavors. I would like to give a special thanks to Mike Miller who has taught me numerous skills over the years starting from my very first Co-op. I would also like to acknowledge the help of Melih Aslan and Ham Rara with reading and editing the thesis and suggesting improvements.

I would like to extend my sincere gratitude and thanks to my advisor and father Dr. Aly Farag who has supported and given me the guidance and "push" needed to always strive for excellence.

Last but certainly not least I would like to thank my parents for their love, support, guidance, tolerance of me and so much more, I dedicate this thesis to them. 


\begin{abstract}
From a computerized image analysis prospective, early diagnosis of lung cancer involves detection of doubtful nodules and classification into different pathologies. The detection stage involves a detection approach, usually by template matching, and an authentication step to reduce false positives, usually conducted by a classifier of one form or another; statistical, fuzzy logic, support vector machines approaches have been tried. The classification stage matches, according to a particular approach, the characteristics (e.g., shape, texture and spatial distribution) of the detected nodules to common characteristics (again, shape, texture and spatial distribution) of nodules with known pathologies (confirmed by biopsies). This thesis focuses on the first step; i.e., nodule detection.
\end{abstract}

Specifically, the thesis addresses three issues: a) understanding the CT data of typical low dose CT (LDCT) scanning of the chest, and devising an image processing approach to reduce the inherent artifacts in the scans; b) devising an image segmentation approach to isolate the lung tissues from the rest of the chest and thoracic regions in the CT scans; and c) devising a nodule modeling methodology to enhance the detection rate and lend benefits for the ultimate step in computerized image analysis of LDCT of the lungs, namely associating a pathology to the detected nodule.

The methodology for reducing the noise artifacts is based on noise analysis and examination of typical LDCT scans that may be gathered on a repetitive fashion; since, a reduction in the resolution is inevitable to avoid excessive radiation. Two optimal filtering methods are tested on samples of the ELCAP screening data; the Weiner and the 
Anisotropic Diffusion Filters. Preference is given to the Anisotropic Diffusion Filter, which can be implemented on $7 \times 7$ blocks/windows of the CT data.

The methodology for lung segmentation is based on the inherent characteristics of the LDCT scans, shown as distinct bi-modal gray scale histogram. A linear model is used to describe the histogram (the joint probability density function of the lungs and nonlungs tissues) by a linear combination of weighted kernels. The Gaussian kernels were chosen, and the classic Expectation-Maximization (EM) algorithm was employed to estimate the marginal probability densities of the lungs and non-lungs tissues, and select an optimal segmentation threshold. The segmentation is further enhanced using standard shape analysis based on mathematical morphology, which improves the continuity of the outer and inner borders of the lung tissues. This approach (a preliminary version of it appeared in [14]) is found to be adequate for lung segmentation as compared to more sophisticated approaches developed at the CVIP Lab (e.g., [15][16]) and elsewhere.

The methodology developed for nodule modeling is based on understanding the physical characteristics of the nodules in LDCT scans, as identified by human experts. An empirical model is introduced for the probability density of the image intensity (or Hounsfield units) versus the radial distance measured from the centroid - center of mass of typical nodules. This probability density showed that the nodule spatial support is within a circle/square of size 10 pixels; i.e., limited to $5 \mathrm{~mm}$ in length; which is within the range that the radiologist specify to be of concern. This probability density is used to fill in the intensity (or Hounsfield units) of parametric nodule models. For these models (e.g., circles or semi-circles), given a certain radius, we calculate the intensity (or Hounsfield 
units) using an exponential expression for the radial distance with parameters specified from the histogram of an ensemble of typical nodules. This work is similar in spirit to the earlier work of Farag et al., 2004 and 2005 [18][19], except that the empirical density of the radial distance and the histogram of typical nodules provide a data-driven guide for estimating the intensity (or Hounsfield units) of the nodule models.

We examined the sensitivity and specificity of parametric nodules in a templatematching framework for nodule detection. We show that false positives are inevitable problems with typical machine learning methods of automatic lung nodule detection, which invites further efforts and perhaps fresh thinking into automatic nodule detection.

A new approach for nodule modeling is introduced in Chapter 5 of this thesis, which brings high promise in both the detection, and the classification of nodules. Using the ELCAP study, we created an ensemble of four types of nodules and generated a nodule model for each type based on optimal data reduction methods. The resulting nodule model, for each type, has lead to drastic improvements in the sensitivity and specificity of nodule detection. This approach may be used as well for classification.

In conclusion, the methodologies in this thesis are based on understanding the LDCT scans and what is to be expected in terms of image quality. Noise reduction and image segmentation are standard. The thesis illustrates that proper nodule models are possible and indeed a computerized approach for image analysis to detect and classify lung nodules is feasible. Extensions to the results in this thesis are immediate and the CVIP Lab has devised plans to pursue subsequent steps using clinical data. 


\section{TABLE OF CONTENTS}

APPROVAL PAGE $\ldots \ldots \ldots \ldots \ldots \ldots \ldots \ldots \ldots \ldots \ldots \ldots \ldots \ldots \ldots \ldots \ldots \ldots \ldots \ldots \ldots \ldots, \frac{\text { Page }}{\text { iii }}$

ACKNOWLEDGMENTS ............................................ iv

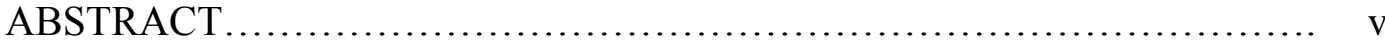

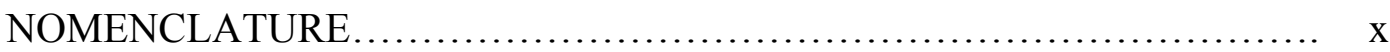

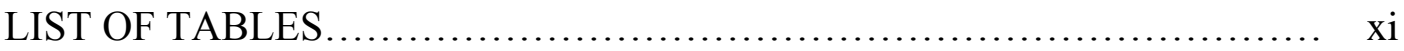

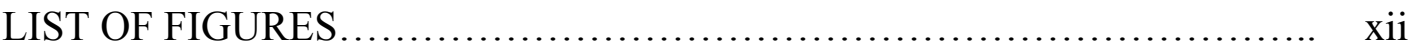

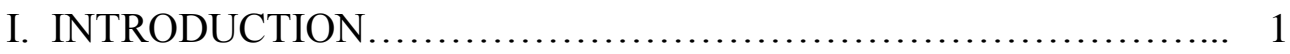

A. Basic Definitions and Scope........................... 1

B. Cancer Research.......................................... 3

C. Lung Screening Trials.................................. 3

D. Nodule Definitions.................................... 9

E. Image Quality Measurements.......................... 11

F. Basic Image Protocols.................................... 12

G. Thesis Focus and Outline............................. 17

H. Summary ............................................ 18

II. IMAGE MODELING ........................................ 19

A. Introduction........................................... 19

B. CT Image Formation.................................. 22

C. Modeling the Uncertainty............................... 27

D. Modeling the Intensity.................................. 44

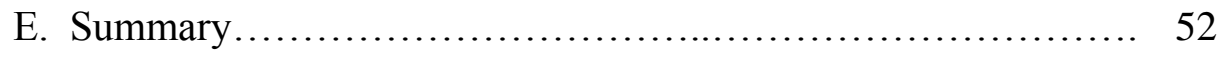


III. IMAGE SEGMENTATION_...................................... 54

A. Introduction................................................ 54

B. Model-Based Lung Segmentation.......................... 57

C. Evaluation of Three Segmentation Algorithm................... 66

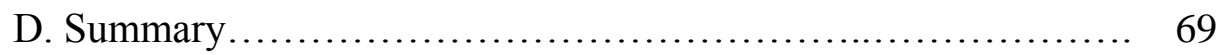

IV. LUNG NODULE MODELING AND AUTOMATIC DETECTION.. 70

A. Introduction.................................................... 70

B. Lung Regions Segmentation.................................... 73

C. Nodule Modeling........................................... 73

D. Nodule Detection............................................. 82

E. Experimental Results........................................ 84

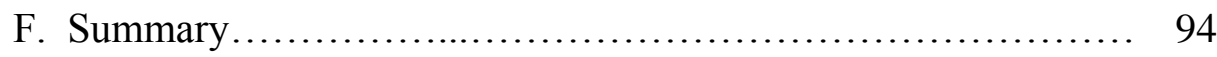

V. NOVEL APPROACH FOR NODULE

MODELING ...................................................... 95

A. Introduction ............................................ 95

B. Data Driven Nodule Modeling............................... 95

C. template Matching............................................ 108

D. Nodule Detection using New Template Method............... 114

E. Experimental Results..................................... 115

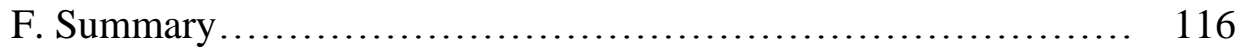

VI. CONCLUSIONS AND RECOMMENDATIONS................... 117

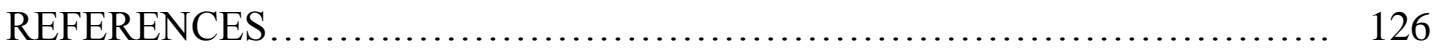

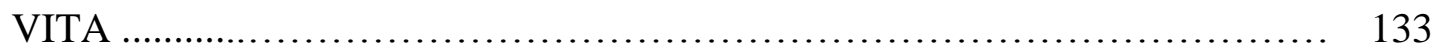




\section{NOMENCLATURE}

\begin{tabular}{|c|c|c|}
\hline$P_{\theta}(t)$ & $=$ & Projection \\
\hline$\delta(\cdot)$ & $=$ & Impulse sheath \\
\hline$\mu$ & $=$ & Mean \\
\hline $\mathrm{N}$ & $=$ & Total number of samples \\
\hline$\sigma$ & $=$ & Standard deviation \\
\hline$C(X, t)$ & $=$ & Diffusion function \\
\hline$\nabla$ & $=$ & Divergence operator \\
\hline $\mathrm{I}(\cdot)$ & $=$ & Image \\
\hline$\theta^{\mathrm{t}}$ & $=$ & Parameter estimate at the $\mathrm{t}$-th iteration \\
\hline$\theta(\cdot)$ & $=$ & Expected value of the complete data log-likelihood \\
\hline$\hat{\mu}_{l}$ & $=$ & Estimated mean of the $1^{\text {th }}$ Gaussian in a Mixture of Gaussians \\
\hline$\hat{\alpha_{l}}$ & $=$ & Estimated weight of the $1^{\text {th }}$ Gaussian in a Mixture of Gaussians \\
\hline$\hat{\Sigma}_{l}$ & $=$ & Estimated covariance of the $1^{\text {th }}$ Gaussian in a Mixture of Gaussians \\
\hline $\mathrm{I}_{\mathrm{p}}$ & $=$ & Voxel intensity \\
\hline$P\left(I_{p} \mid f_{p}\right)$ & $=$ & Marginal density \\
\hline$\gamma$ & $=$ & Gibbs potential \\
\hline$\Phi(.,)$. & $=$ & Gaussian density \\
\hline $\mathrm{p}(\cdot)$ & $=$ & Probability density function \\
\hline
\end{tabular}




\section{LIST OF TABLES}

I. The Sum Square Difference (SSD) measure with respect to the ground truth

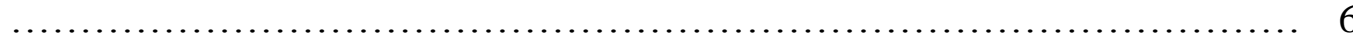

II. The Mutual Information (MI) measure with respect to the ground truth

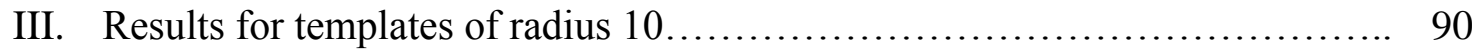

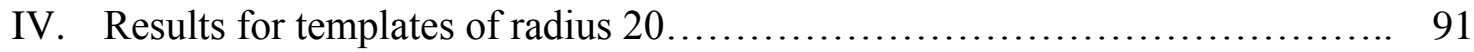

V. Results of template matching (single sweep) using the new template design

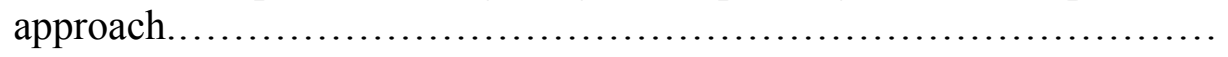

VI. Results of template matching (single sweep) using the new template design approach for four orientations per nodule type, using step size 45 (total of 16

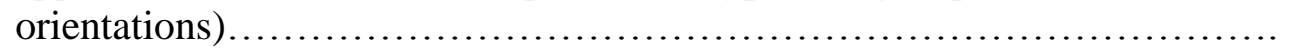




\section{LIST OF FIGURES}

1 A slice of a CT scan obtained from a human subject. Each pixel resembles an area of the lung tissue of about $3 \mathrm{~mm}$. Better imaging resolution is possible at the expense of larger radiation exposure and scanning time.

2 An ensemble of the four classes of pulmonary nodules

3 Sample slices from the ELCAP study. Note the severe degradation of image quality and the ambiguity of the nodules. The dots show the location of the nodules as determined by human expert $[5]$

4 Illustration of the segmentation process. Left: a CT slice from a thoracic scan. Right: segmentation output, showing the isolated lung tissue.

5 A block diagram of the major steps involved in computer-based analysis of LDCT of the chest in order to detect and classify doubtful lung nodules.

Illustration of parallel beam projections where a set of rays hit an object

$6 \mathrm{~g}(\mathrm{x}, \mathrm{y})$ at an angle $\mathrm{P}_{\boldsymbol{\theta}}(\mathrm{t})$ per angle $\boldsymbol{\theta}$. On the right we highlight the parameters relating one ray $(\mathrm{AB})$ to the projection process.

7 Illustration of reconstruction from one projection of the Shepp-Logan model [29] implemented using MatLab. The inverse Fourier transform

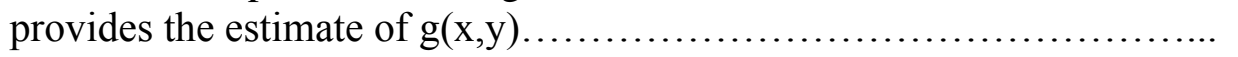

8 Examples of high and low resolution thoracic scans...................

9 Modeling the observed image as an ideal image $s(m, n)$ disturbed with an additive noise $\eta(m, n)$. The Weiner filter is an operator $h(m, n)$ that provides an estimate for $\mathrm{s}(\mathrm{m}, \mathrm{n})$ in the mean square error sense...........

10 A sample of 16 slices from a patient scan of the ELCAP study

11 Histogram of the slices in Figure 10. Note the distinct bimodal nature of the histograms.

12 Cropped noise background of the 16 slices in Figure 10. For Visualization purposes the images are placed on a white background 
13 The histogram of the noise from the cropping operation

14 Average noise histogram obtained from 16 slices

15 The histogram of the noise from the cropping operation resulting from 16 full scans of the ELCAP data. Each histogram is the average of all the histograms corresponding to cropping the noise off all the slices in the 16 scans

16 Average noise histogram obtained from 16 scans

17 Wiener filter applied onto four original images with different window sizes. The first row represents the original images, second row is the wiener filter applied with window [3 3], third row is with window [7 7], fourth row is with window [9 9], fifth row is with window [11 11] and the last row is window size [511 511].

18 Anisotropic filter for different kappa values. The first row are the original images for four original images, second row is the filtered image with $\mathrm{k}=($ standard deviation of intensity $-\mathrm{std}-$ values in a slice $) / 511$, third row $\mathrm{k}=($ std of slice $) / 21$, fourth $\mathrm{k}=($ std of slice $) / 11$, fifth row $\mathrm{k}=($ std of slice $) / 9$ and the last row $\mathrm{k}=($ std of slice $) / 3$

19 Performance of the adaptive Weiner filter on data sets of known nodule locations. From left to right: original slices, adaptive Weiner filtering of size $3 \times 3,5 \times 5$ and $7 \times 7$. (Red circle shows the nodule location)

20 Performance of the anisotropic diffusion filter on data sets of known nodule locations. From left to right: original slices, anisotropic diffusion filtering of three different $\mathrm{k}$ parameters $(\mathrm{k}=$ standard deviation $/ \mathrm{N} ; \mathrm{N}=5$, 8,10 respectively). (Red circle shows the nodule location)

21 A Fitting a linear model to the pdf of a typical lung CT scan................

22 Average histogram of a CT slice from the ELCAP dataset. The average histogram of CT slices of the ELCAP database. Lung region (parenchyma) and fat/muscle region constitute two dominant peaks in the histogram, in order to separate the lung region a threshold is chosen to maximize the separation between these two peaks

23 Block diagram of the segmentation algorithm. 
24 Output of the segmentation algorithms with respect to ground truth on sample slices from the ELCAP dataset. From left to right: Original, Manual Segmentation; Algorithm 1, Algorithm 2 and Algorithm $3 . .$.

25 A block diagram of the major steps involved in computer-based analysis of LDCT of the chest in order to detect and classify doubtful lung nodules

26 An ensemble of the four classes of pulmonary nodules segmented by experts.

27 The distribution of the radial distance from the centroid of the nodules. Note the standard exponential behavior of the radial distance. This will be used in the design of templates as we will discuss in the following section.. 75

28 Shows the average distribution of $\mathrm{HU}$ densities for each nodule type.

29 Histogram of the Juxta nodule type................................. 77

30 Histogram of the Well-Circumscribed nodule type..................... 78

31 Histogram of the Vascular nodule type............................... 78

32 Histogram of the Pleural Tail nodule type.......................... 79

33 Probability density of the Radial distance of the Pleural Tail nodule. Note that the density is concentrated for distances less than 5 pixels from the centroid.

34 Probability density of the Radial distance of the Vascular nodule

35 Probability density of the Radial distance of the Well-Circumscribed nodule

36 Probability density of the Radial distance of the Juxta nodule...

37 Average radial distance of four nodule types....

38 Samples of parametric templates of various sizes in 2D and 3D...

39 Normalized Cross-Correlation (NCC) for circular templates on regions around the correct nodule location. The figure is the average NCC for all slices having the same nodule type. Upper left is the average NCC for 
Pleural-Tail nodules, Upper right is the results for Vascular nodules, lower left is the results for Well-Circumscribed nodules, and Lower right shows the results for Juxta-Pleural nodules. The location of the nodules (as provided by the ELCAP experts), shown in black, may not correspond to the center or centroid of the real nodules..................................

40 Distribution of the Normalized Cross-Correlation (NCC) for parametric nodules (circular and semi-circular) with radius 10 pixels. Higher NCC values results in less FPs while smaller values provide more FPs. In the ROC plots (Figures 39- 42) a NCC threshold of 0.5 was selected...........

41 ROC curve of different template shape applied over well circumscribed nodules...

42 ROC curve of different template shape applied over juxta-pleural nodules.. 92

43 ROC curve of different template shape applied over vascular nodules...... 93

44 ROC curve of different template shape applied over pleural-tail nodules....

45 Procedure to generate data-driven nodule model.

47 Two shapes (together with the control points) used for the shape warping numerical example...

Forward-map image warping. (a) Source (containing $\mathbf{x}_{\mathbf{i}}$ ) and destination (containing $\left.\mathbf{x}_{\mathbf{i}}^{\prime}\right)$ images. $\left(\mathrm{u}_{\mathrm{i}}, \mathrm{v}_{\mathrm{i}}\right)$ are integer values and $\left(\mathrm{x}_{\mathrm{i}}, \mathrm{y}_{\mathrm{i}}\right)$ are real values. (b) Apply forwardF-map f to all source image pixels. (c) Many source pixels can map to the same destination pixel. (d) Some destination pixels may not be even covered.

49 Reverse-map image warping. (a) Source (containing $\mathbf{x}_{\mathbf{i}}$ ) and destination (containing $\left.\mathbf{x}_{\mathbf{i}}^{\prime}\right)$ images. $\left(\mathrm{u}_{\mathrm{i}}, \mathrm{v}_{\mathrm{i}}\right)$ are real values and $\left(\mathrm{x}_{\mathrm{i}}, \mathrm{y}_{\mathrm{i}}\right)$ are integer values. (b) Iterate over the destination image. It is necessary to resample source (e.g., bilinear, cubic interpolation). Notice that all pixels in the destination image can be easily determined after resampling, unlike in Figure 48. 
50 Image warping numerical example illustration. (a) Source image with pixel values and control points. (b) Destination image with unfilled pixel values and control points. (c) The actual destination image after warping the source image, according to the control points

51 Generating the Juxta nodule. (a) Original (non registered nodules); (b) manually annotated nodules; (c) co-registered (with respect to first nodule) nodules; and (d) average nodule with and without registration. Notice the averaging process creates a nodule which maintains the main characteristics of Juxta nodules...

52 Generating the Well-Circumscribed nodule. (a) Original (non registered nodules); (b) manually annotated nodules; (c) co-registered (with respect to first nodule) nodules; and (d) average nodule with and without registration. Notice the averaging process creates a nodule which maintains the main characteristics of Well-Circumscribed nodules...........

53 Generating the Vascular nodule. (a) Original (non registered nodules); (b) manually annotated nodules; (c) co-registered (with respect to first nodule) nodules; and (d) average nodule with and without registration. Notice the averaging process creates a nodule which maintains the main characteristics of Vascular nodules.

54 Generating the Pleural Tail nodule. (a) Original (non registered nodules); (b) manually annotated nodules; (c) co-registered (with respect to first nodule) nodules; and (d) average nodule with and without registration. Notice the averaging process creates a nodule which maintains the main characteristics of Pleural Tail nodules

55 Distribution of the Normalized Cross-Correlation (NCC) for nonparametric templates. Higher NCC values results in less FPs while smaller values provide more FPs.

56 Normalized Cross-Correlation (NCC) for circular templates on regions around the correct nodule location. The figure is the average NCC for the pleural tail nodule type. Upper left is the average NCC for Pleural-Tail nodule using pleural tail template, Upper right is the results for PleuralTail nodule using Vascular template, lower left is the results for PleuralTail nodule using Well-Circumscribed template, and Lower right shows the results for Pleural-Tail nodule using Juxta-Pleural template. The location of the nodules (as provided by the ELCAP experts), shown in black, may not correspond to the center or centroid of the real nodules...... 
57 Normalized Cross-Correlation (NCC) for circular templates on regions around the correct nodule location. The figure is the average NCC for the Vascular nodule type. Upper left is the average NCC for Vascular nodule using pleural tail template, Upper right is the results for Vascular nodule using Vascular template, lower left is the results for Vascular nodule using Well-Circumscribed template, and Lower right shows the results for Vascular nodule using Juxta-Pleural template. The location of the nodules (as provided by the ELCAP experts), shown in black, may not correspond to the center or centroid of the real nodules. Block diagram of novel nodule detection design

58 Normalized Cross-Correlation (NCC) for circular templates on regions around the correct nodule location. The figure is the average NCC for the Well-Circumscribed type. Upper left is the average NCC for WellCircumscribed nodule using pleural tail template, Upper right is the results for Well-Circumscribed nodule using Vascular template, lower left is the results for Well-Circumscribed nodule using Well-Circumscribed template, and Lower right shows the results for Well-Circumscribed nodule using Juxta-Pleural template. The location of the nodules (as provided by the ELCAP experts), shown in black, may not correspond to the center or centroid of the real nodules..............................

59 Normalized Cross-Correlation (NCC) for circular templates on regions around the correct nodule location. The figure is the average NCC for the Juxta type. Upper left is the average NCC for Juxta nodule using pleural tail template, Upper right is the results for Juxta nodule using Vascular template, lower left is the results for Juxta nodule using WellCircumscribed template, and Lower right shows the results for Juxta nodule using Juxta-Pleural template. The location of the nodules (as provided by the ELCAP experts), shown in black, may not correspond to the center or centroid of the real nodules .............................

60 Block diagram of novel nodule detection design 


\section{CHAPTER 1}

\section{INTRODUCTION}

\section{A. Basic Definitions and Scope}

This thesis deals with image analysis of chest low dose CT (LDCT) scans for early detection of doubtful nodules in the lung tissues that may provide a clue for potential lung cancer. The term cancer is a horrifying one; yet it is a technical term and refers to uncontrolled cell growth in tissues leading to disturbances of the functions of the organs of the body and, at extreme state of influence, cause major suffering and even death. On the website of the National Cancer Institute (NCI) http://www.cancer.gov/cancertopics/what-is-cancer [1]: "Cancer is a term used for diseases in which abnormal cells divide without control and are able to invade other tissues. Cancer cells can spread to other parts of the body through the blood and lymph systems. Cancer is not just one disease but many diseases. There are more than 100 different types of cancer. Most cancers are named for the organ or type of cell in which they start - for example, cancer that begins in the colon is called colon cancer; cancer that begins in basal cells of the skin is called basal cell carcinoma. Cancer types can be grouped into broader categories. The main categories of cancer include:

- Carcinoma - cancer that begins in the skin or in tissues that line or cover internal organs. 
- Sarcoma - cancer that begins in bone, cartilage, fat, muscle, blood vessels, or other connective or supportive tissue.

- Leukemia - cancer that starts in blood-forming tissue such as the bone marrow and causes large numbers of abnormal blood cells to be produced and enter the blood.

- Lymphoma and myeloma - cancers that begin in the cells of the immune system.

- Central nervous system cancers - cancers that begin in the tissues of the brain and spinal cord" [1].

The National Institute of Health (NIH) lists on its website www.nih.gov [2] various types of organ cancers; the common ones are: Bladder Cancer; Breast Cancer; Colon and Rectal Cancer; Endometrial Cancer; Kidney (Renal Cell) Cancer; Leukemia; Lung Cancer; Melanoma; Non-Hodgkin Lymphoma; Pancreatic Cancer ; Prostate Cancer; Skin Cancer (Nonmelanoma); and Thyroid Cancer.

In 2008, there were $1,437,180$ new cases of cancer reported in the United States: (does not include nonmelanoma skin cancers), and 565,650 cancer related deaths. Due to the impact of cancer on our society, cancer research occupies major focus of the research community, with the annual budget of United States' National Cancer Institute (NCI) for FY 2008 totaling $\$ 4.83$ billion. For FY 2007, it was $\$ 4.79$ billion, and, for FY 2006, it was $\$ 4.75$ billion - roughly $16 \%$ of the NIH budget (about $\$ 29$ billion) is spent on cancer related research [1][2]. Indeed, cancer is a global problem.

This thesis is in the realm and domain of lung cancer. The NIH [2] defines lung cancer as "Cancer that forms in tissues of the lung, usually in the cells lining air passages. 
The two main types are small cell lung cancer and non-small cell lung cancer. These types are diagnosed based on how the cells look under a microscope." Lung cancer numbers are higher than any other type of cancer. There was an estimated 215,020 new cases and 161,840 deaths from lung cancer (non-small cell and small cell combined) in the United States in 2008 [1][2].

\section{B. Cancer Research}

Suffices to say, that enormous efforts are exerted around the clock in every corner of the globe to combat cancer. Research is conducted by the cellular level, by genetics background, by tissue type and by demographic factors. Efforts for diagnosis and treatment are ongoing and new technologies are employed by the governmental agencies as well as the private sector. There are cancer centers elsewhere that deal with generic cancer research as well as the regional factors. Computational methods are an integral part of all research efforts on cancer. Image analysis approaches have been used for decades to analyze microscopic images, X-ray scans and CT scans. This thesis is concerned with macro scale imaging (at the gross anatomical level of the lung tissues). The resolution of the imaging system enables tissue-level examination. Figure 1 shows an imaging scan calibrated with respect to the physical dimensions of a human subject.

\section{Lung Screening Trials}

Screening studies for early detection of lung cancer using X-ray, CT and sputum analysis have been conducted worldwide during the past three decades. Since the mid-1990s, a series of non-randomized single-arm studies, have shown low-dose CT (LDCT) to be 
more sensitive than standard chest radiography for detecting small non-calcified nodules $(\mathrm{NCN})$.

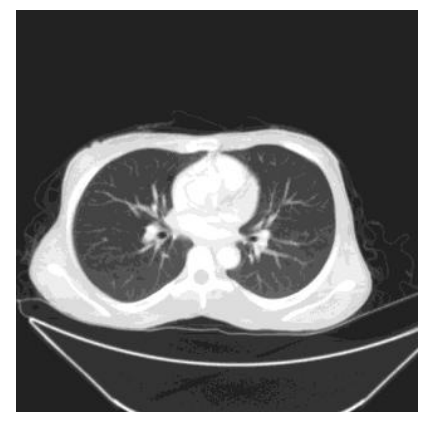

Figure 1: A slice of a CT scan obtained from a human subject. Each pixel resembles an area of the lung tissue of about $3 \mathrm{~mm}$. Better imaging resolution is possible at the expense of larger radiation exposure and scanning time.

Below, we highlight some of the national screening studies; as described in the article of

\section{LaRocca et al. [4].}

\section{i. $\quad$ Early Lung Cancer Action Project (ELCAP) [5]}

Early Lung Cancer Action Project (ELCAP) selected 1000 patients of age greater than 60 , with a minimum of 10 pack per year history of cigarette smoking, to undergo CT chest scans. A $23 \%$ detection rate for solitary NCN was reported. Of these NCN, 12\% were later determined to be malignant. The cancer detection rate was much greater than chest radiography alone and statistically significant [6].

A nonrandomized Mayo Clinic cohort study of 1520 individuals who underwent three annual low dose CT examinations also confirmed that low dose spiral CT is superior to chest radiography in detecting NCNs and early stage lung cancer [7]. A series of Japanese studies using similar screening parameters reached the same conclusion. 
Single arm studies are prone to biases such as lead-time, length and over diagnosis when calculating for 5-year survival. However, recent advances in CT imaging technology regarding slice thickness, and superior resolution have increased the detection rate of NCN. Given this higher resolution LDCT, the majority of identified NCN are smaller than 5mm in size [8]. The International-ELCAP (I-ELCAP) published guidelines for assessing NCN detected by LDCT. The guidelines have evolved, and are based primarily on size and consistency of the nodules. The I-ELCAP algorithm further defined a subsequent evaluation of NCN and criteria to seek tissue acquisition [9]. The newer guidelines were based on the percent volume change and the monthly volumetric growth index from the original 94 nodules presented by the ELCAP group [10].

\section{ii. National Lung Screening Trail (NLST) [2]}

As advertised on the NCI website [2], "the National Lung Screening Trial (NLST) is a lung cancer screening trial sponsored by the National Cancer Institute (NCI). Screening means testing people to detect a disease before it causes symptoms. Launched in 2002, NLST is comparing two ways of detecting lung cancer: spiral computed tomography (CT) and standard chest X-ray. Both chest X-rays and spiral CT scans have been used to find lung cancer early. So far, neither chest X-rays nor spiral CT scans have been shown to reduce a person's chance of dying from lung cancer. This study will aim to show if either test is better at reducing deaths from this disease. By February 2004, nearly 50,000 current or former smokers had joined NLST at more than 30 study sites across the country. The trial, now closed to further enrollment, is slated to collect and analyze data for eight years, and will examine the risks and benefits of spiral CT scans compared to 
chest X-rays. This trial is a randomized, controlled study--the "gold standard" of research studies--and is large enough to determine if there is a 20 percent or greater drop in lung cancer mortality from using spiral CT compared to chest X-ray.”

In justification of the NLST study, the investigators state [2] "Currently, when lung cancer is detected, the disease has already spread outside the lung in 15 percent to 30 percent of cases. Spiral CT, a technology introduced in the 1990s, can pick up tumors well under one centimeter $(\mathrm{cm})$ in size, while chest X-rays detect tumors about 1 to $2 \mathrm{~cm}$ ( 0.4 to 0.8 inches) in size. Conventional wisdom suggests that the smaller the tumor, the more likely the chance of survival. But no scientific evidence to date has shown that screening or early detection of lung cancer actually saves lives. NLST, because of the large number of individuals participating and because it is a randomized, controlled trial, will be able to provide the evidence needed to determine whether spiral CT scans are better than chest X-rays at reducing a person's chances of dying from lung cancer.”

To date there has been no report regarding the analysis of the NLST data to indicate whether any of the stated goals have been achieved.

iii. The Louisville Lung Screening Study [3][4]

The Louisville Lung Cancer Study was a randomized prospective pilot study comparing annual chest $\mathrm{x}$-rays to annual LDCT in individuals at high-risk for the development of lung cancer in a community setting. Individuals were determined to be at high-risk for the potential development of lung cancer by use of a Risk Assessment Questionnaire, and baseline spirometry. Inclusion requirements included a life expectancy of at least 5 years based on no co-morbid conditions, no other history of 
cancer other than non-melanotic skin cancer or cervical carcinoma in-situ (CIS). Using a randomized block design, 500 individuals with a normal (or abnormal but stable) E-cxr were randomized to receive a baseline low-dose spiral CT of the chest (LDCT-0) within 2-4 weeks of E-cxr and then yearly for three years (LDCT-1, LDCT-2 and LDCT-3), and 500 were randomized to a repeat annual chest x-ray for three consecutive years (CXR-1, CXR-2 and CXR-3).

All CT scanning was performed with either a Picker PQ 6000 single-slice helical scanner or a General Electric Lightspeed 4-slice multi-slice scanner, both having slip-ring scanning capabilities with a slice thickness during acquisition of $8 \mathrm{~mm}$ reconstructed every $4 \mathrm{~mm}$. Images were displayed in lung windows only, using an edge-enhancing bone algorithm. The scanning pitch was 1.5. Images were interpreted on a digital workstation. If repeat imaging was required, thin section imaging was repeated but with a slice thickness of 1.0 or $1.5 \mathrm{~mm}$.

The screening algorithm for assessing abnormal x-rays was as follows [4]: All chest radiographs were reviewed on film or soft copy workstations. If a new abnormality was identified on annual scan, then a high resolution chest CT scan would be performed. If the CT scan was suspicious for a neoplasm then tissue acquisition was undertaken by the pulmonologist or thoracic surgeon. The algorithm for abnormal LDCT findings was the same and formulated from the guidelines for diagnostic evaluation established by ELCAP [5][6]. Normal, benign or unchanged baseline and repeat studies were requested to return for annual screening $\mathrm{CT}$ as was the protocol for normal E-cxr. For suspicious or new masses $>10 \mathrm{~mm}$ in diameter, tissue acquisition (open resection, needle biopsy, 
bronchoscopy, or thoracoscopy) was pursued. NCN 5-10mm in diameter were screened with high-resolution, thin-section CT imaging. If the screening was determined to be benign, the subject was returned to routine screening protocol. For suspicious NCN between 5-10mm due to speculation etc., the patient was referred for tissue acquisition. Indeterminate nodules on high-resolution imaging were re-imaged in 3 months. If the nodules remained unchanged, the subject was re-imaged 9 months later and returned to the annual group. If the nodule changed or increased after 3 months, tissue acquisition was requested. The protocol was followed for 3 consecutive years with annual repeat imaging per randomization. Individuals were then followed for an additional two years (OBs-4 and OBs-5).

A final analysis was conducted by contacting all participants that signed an informed consent and underwent an E-cxr ( $\mathrm{n}=1057)$. In addition, mortality was determined using the Social Security Death Index (SSDI), as accessed from a free web site. The ability to determine death accurately using the SSDI is reported to be $91 \%$ sensitive (95\% CI, 84\%$95 \%$ ) and $87 \%$ specific $(95 \%$ CI, 47-99\%) The final date of analysis was determined to be February 2007 (5 years after the enrollment of the final participant). From this data, overall survival for the two arms, and lung cancer specific mortality were estimated [4].

Conclusion of the analysis of the Louisville screening study suggests that annual lung cancer screening can be used to target a high-risk population of smokers with airflow obstruction. Baseline LDCT screening for lung cancer resulted in a larger proportion of early-stage diagnoses than annual CXR. Lung cancer-specific mortality may be less in the CT arm but we must await the results of larger studies powered to show this 
difference if it truly exists. Attrition from study protocols and adherence to re-scanning design are difficult to control in a community-based study [4].

From the image analysis prospective, while reasonable expectations (conventional wisdom) would suggest that CT is better than X-ray vis-à-vis diagnosis, no telling as yet whether early detection using CT improves mortality rate (i.e., reduction of cancerrelated death once detected). The conventional wisdom is supported, from imaging point of view, as CT would contain more information than X-ray; i.e., the ability to see more in an image will lead to better diagnosis of doubtful nodules. Of course, CT carries the risk of more radiation and cost [4].

\section{Nodule Definitions}

A pulmonary nodule usually has a spherical shape; however, it can be perplexed by surrounding anatomical structures such as vessels and the pleural surface. Nodules, as observed in a CT slice, may appear at various locations in the lung tissues, and may take various size and shape. Kostis et al. [12] classify nodules into four classes:

- well-circumscribed where the nodule is located centrally in the lung without being connected to vasculature;

- vascularized where the nodule has significant connection(s) to the neighboring vessels while located centrally in the lung;

- $\quad$ pleural tail where the nodule is near the pleural surface, connected by a thin structure; and

- juxta-pleural where a significant portion of the nodule is connected to the pleural surface. 
See Figure 2 for illustration.
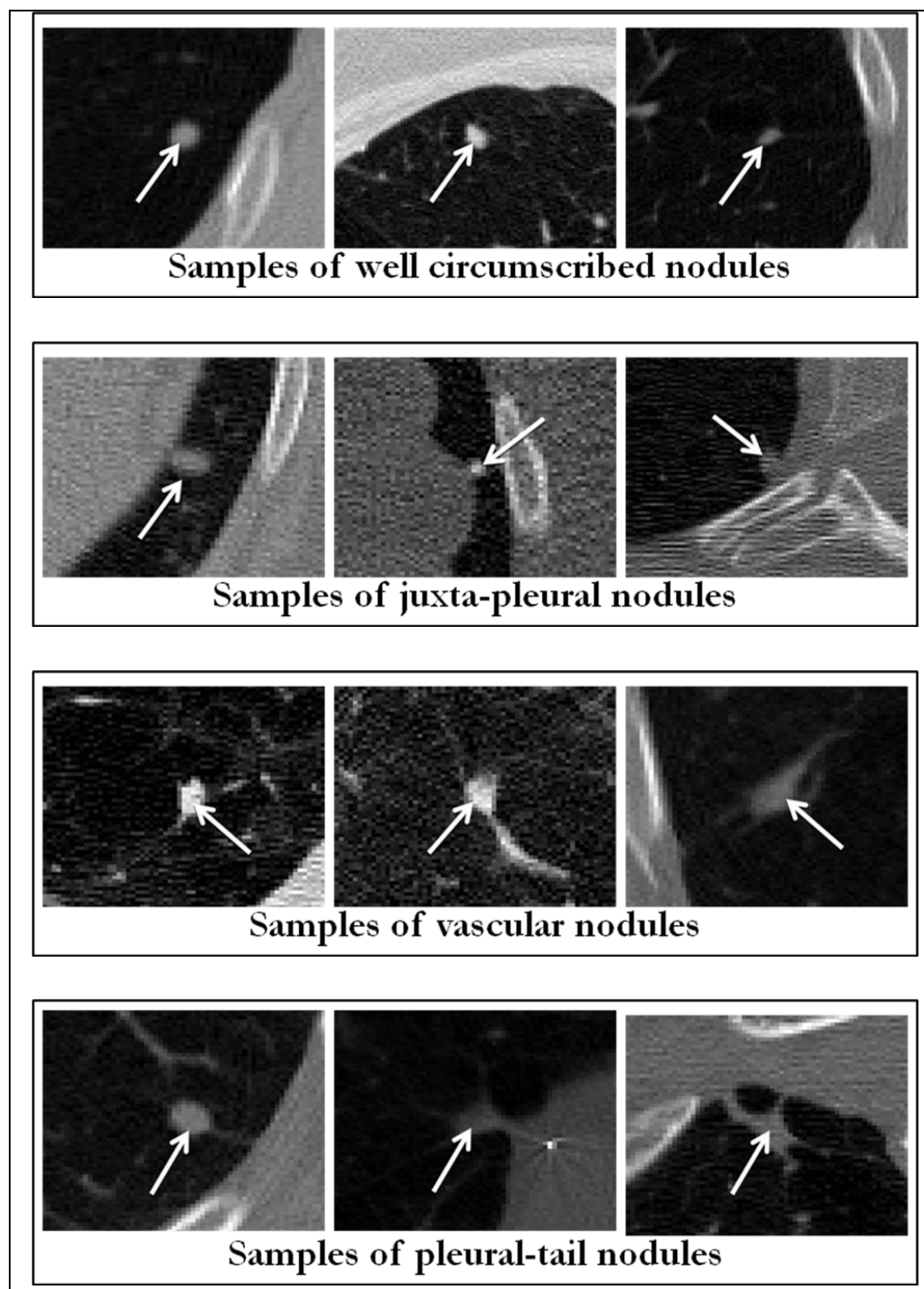

Figure 2: An ensemble of the four classes of pulmonary nodules [12]. 


\section{E. Image Quality Measurements}

The quality of the CT scan obtained from the imaging protocol affects the results of subsequent analysis. As computerized methods attempt to mimic the physician, if the human expert cannot observe the information about the nodule, the computer will not detect it either. This goes with the general rule of image analysis, if the information is missing one cannot regain it; missing mean cannot be observed. From standpoint of image quality, several measures have been used including:

Image Resolution: It is the scale that corresponds pixel size in an image (e.g., CT slice) with respect to physical tissue dimensions. Image scanners provide this information with respect to physical body anatomy.

Image Contrast: It is the scale that measures the distinction between image components. Imaging protocols may be designed to artificially enhance the region separability or contrast. In image analysis, contrast refers to appearance differences between classes of objects.

Image Sensitivity: Refers to ability of the imaging protocol to enhance the contrast between anatomical features and non-anatomical features (e.g., ability to discriminate between nodules and blood vessels or alveolar branches and sacks in a lung tissue).

Image Specificity: Refers to ability to distinguish pathologies from image information.

The sensitivity and specificity terms are generic and the definitions may describe an image or the outcome of an algorithm. 


\section{$\underline{\text { F. Basic Image Protocols }}$}

Biomedical image analysis as applied to nodule detection and classification is a very rich area in the computational literature. Sluimer et al. [13] provides a survey of computational studies on automatic detection of lung nodules before 2006. The common thread of these approaches may be summarized in the following steps:

\section{Image Processing}

It is often the case that the CT scan of a particular patient suffers from various uncertainties or errors. Uncertainties in a CT scan may include spurious noise, motion artifact, and low contrast. Image processing tools are employed to correct for such errors. Some of these tools may be mundane such as applying a median filter to remove spurious noise or involved such applying scale space methods or an optimal filter to reduce the noise while keeping the anatomical features and the nodules. Likewise, basic normalization may be used to correct for motion artifacts and for proper referencing of subsequent scans. It is virtually impossible to list all the image processing tools that may be employed for conditioning the CT scans before further analysis. With high resolution scans and calibration of the CT scanner and rigid adherence to the imaging protocol, minimal processing may be necessary. Unfortunately, some clinical studies (e.g., ELCAP [5]) have various uncertainties and significant degradations in image quality to necessitate significant processing as will be described in this thesis.

Figure 3 shows sample of CT slices to be used in this thesis from the ELCAP screening study. 


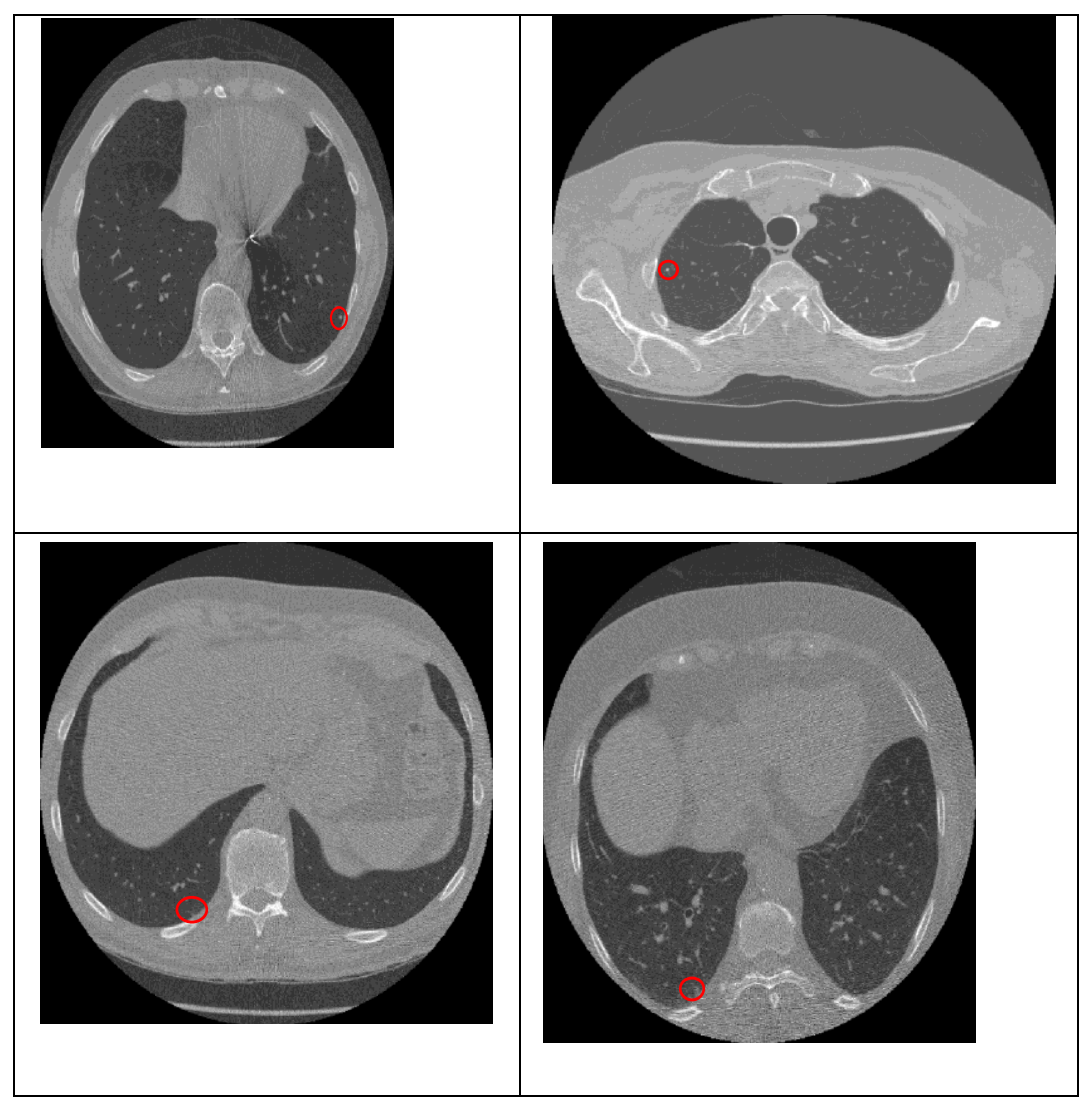

Figure 3: Sample slices from the ELCAP study. Note the severe degradation of image quality and the ambiguity of the nodules. The dots show the location of the nodules as determined by human expert [5].

In our work, we employed the Wiener and Anisotropic diffusion filtering approaches to reduce the noise effect.

\section{Image Segmentation}

Image segmentation is a fundamental step in computerized image analysis and it deals with separating classes in an image into continuous and separate regions. For example, a 
CT slice from a thoracic (e.g., Figure 4(left)) scan may contain chest wall, heart segments in addition to lung tissues. The goal of segmentation in this case is to isolate the lung tissue (Figure 4(right)).
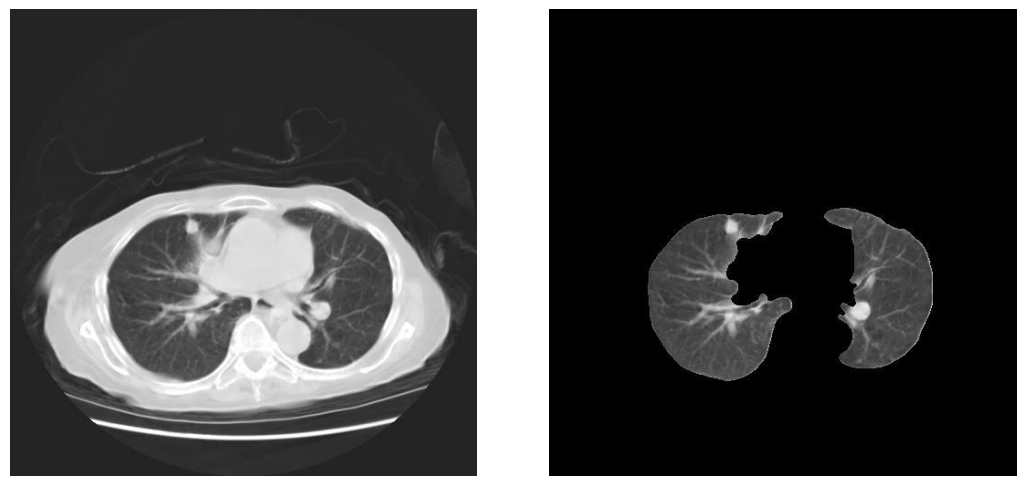

Figure 4: Illustration of the segmentation process. Left: a CT slice from a thoracic scan. Right: segmentation output, showing the isolated lung tissue.

As in image processing, image segmentation is extremely rich area in the image analysis and computer vision literature. Yet, broadly speaking, image segmentation methods may be classified into three distinct approaches, a) statistical, b) geometric, and c) variational. Statistical methods model the image information and cast the region process as mapping from raw images. Geometric methods exploit object shape descriptions in order to separate the image contents into classes. Variational methods (e.g., level sets) create an implicit description of class boundaries in terms of a curve/surface that evolves and cuts the particular object at its boundary (zero-level set). Variational approaches uses implicit models (e.g. level sets), and explicit models (e.g., 
snakes and gradient vector flow). Under each category, there exists an enormous body of theoretical and algorithmic foundation that is well beyond this thesis.

In terms of segmenting the CT scans, we describe an approach that is based on modeling the intensity information in the CT slices using the linear model [14]. This method is evaluated against two sophisticated approaches developed in the CVIP Lab based on the level sets [15] and random fields [16], and is shown to provide comparable performance yet is faster and requires minimal human intervention.

\section{Nodule Detection}

The process of module detection involves nodule modeling and an approach to distinguish the nodules from the anatomical structure in the lung tissue [17]. Even though not necessary, nodule detection is usually applied to the lung tissue after the segmentation step. This approach will ignore the rest of the chest and thoracic regions, which may contain nodules as well. Since our focus is on lung cancer, we will always apply the nodule detection step after the segmentation of the lung region.

A crucial component of nodule detection is nodule modeling. We examine in this thesis data-driven strategies for nodule modeling. The approach depends on estimating the gray level distribution of a template model using an ensemble of nodules collected by manual segmentation by an expert. 


\section{Nodule Classification}

Nodule classification involves assigning pathology to the detected and isolated nodules. This is the ultimate goal of computerized nodule detection for early detection of doubtful nodules. The success of this step hinges on availability of a real discriminatory database of malignant and benign nodules that are adequate for designing and testing a classifier. At the writings of this thesis, such data is not available to provide the necessary testing and validation. Therefore, the thesis will focus on the detection step.

\section{The CVIP Lab Approach}

The CVIP Lab has developed an approach for nodule detection based on analytical foundation (e.g., [18-20]. The classification approach has only been conceptualized on a few data sets. It has not been tested nor validated on a significant clinical study as of now. An IRB establishing a collaboration with the Jewish Hospital Foundation on "Nodule Detection and Classification from Chest Low Dose CT (LDCT) Scans" has just been approved at the conclusion of this thesis, which will enable accessibility of clinical data from the Louisville Screening Study [3][4], and will enable further validation of the detection step and the execution of the classification step. 


\section{G. Thesis Focus and Outline}

From the above discussion, the generalized framework for a computerized image analysis for detection and classification of LDCT consists of four main steps (see, Figure 5): 1) Filtering and normalization of the LDCT scans; 2) Segmentation of the lung regions (parenchyma) from the surrounding tissue; 3) Detection of lung nodules; and 4) Nodule classification as benign or malignant.

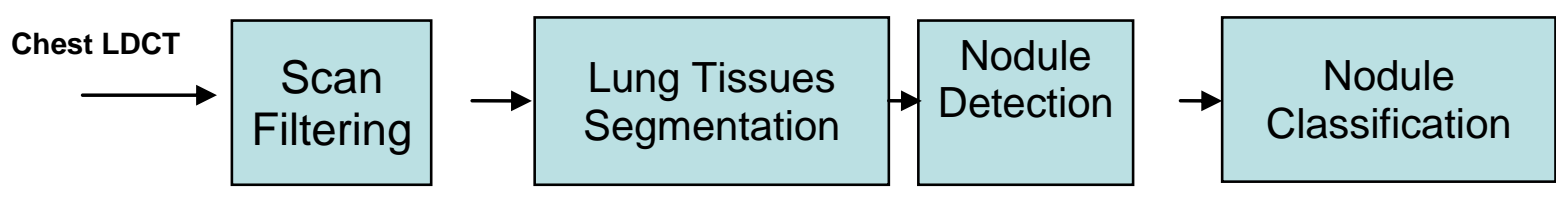

Figure 5: A block diagram of the major steps involved in computer-based analysis of LDCT of the chest in order to detect and classify doubtful lung nodules.

This thesis focuses on the first three blocks of the computerized system. The testing of the methodologies has been entirely on the ELCAP data obtained from the study website [5]. The major contributions of the thesis are the following: a) evaluation of the sources of uncertainty in the LDCT data to properly reduce their effects; b) establishing a reliable and robust approach (in terms of speed and accuracy) for lung tissue segmentation; and c) establishing a data-driven nodule modeling approach that provides acceptable levels of performance of nodule detection, with respect to human experts, as measured in terms of sensitivity and specificity of the lung nodules visible in chest LDCT scans. 


\section{H. Summary}

This chapter examined the domain of the problem under investigation and the basic issues related to image analysis for early detection of lung cancer. The rest of the thesis is organized as follows:

Chapter 2 focuses on image modeling approaches used for processing and normalization of the lung tissues from a CT scan.

Chapter 3 describes the segmentation algorithm and evaluates its performance with respect to some homegrown approaches developed at the CVIP Lab, in particular the algorithms of Farag and Abdelmunim, 2004 [15] and Ali and Farag, 2008 [16].

Chapter 4 tackles the problem of nodule detection and studies the sensitivity and specificity of nodule detection. The evolution will be based on the ELCAP data. This chapter builds on our published work in 2008 [14].

Chapter 5 introduces a novel approach for nodule modeling that takes into account the inherent characteristics of physical nodules. The design methods are examined and the improvements in sensitivity and specificity of the detection approach over traditional methods are explained.

Finally, Chapter 6 provides conclusions gained from this research and suggestion for future work. 


\section{CHAPTER 2}

\section{IMAGE MODELING}

\section{A. Introduction}

Image modeling is a branch of signal and image analysis which deals with developing mathematical description of the information captured in an image. The first step for proper image modeling is to understand the image formation process; thus the knowledge of what an image supposes to represent is the stepping stone for subsequent steps. As images and signals are generated by various sources, one can expect the modeling to be different. Hence, modeling an image captured by a CCD camera is certainly different than modeling an image captured by an X-ray or CT imaging process. In the first case, the parameters of the camera, the object, and the light source determine the image capturing process; i.e., image formation is mainly an optical process. In the x-ray case, it is the attenuation pattern of high energy rays (X-rays) captured by a film or a detector that describes an image; i.e., image formation is a physical and mathematical processes. In CT imaging, rays penetrating a portion of an object (e.g., slap/slice of the human body) are captured at the other end by detectors, and through the Fourier slice theorem, the received energy is back-projected to get an estimate of the structure of the object in the path of the rays; i.e., here too, the image formation is both physical and mathematical [21][22]. Similarly, image formation in ultrasound is physical and mathematical; in magnetic resonance imaging (MRI) image formation is mathematical, etc. Understanding 
how an image is generated enables proper modeling of sources of uncertainty, and hence, recovering the original (most accurate form) of the image [23].

At the algorithmic level, it is often the case that the image formation process is either forgotten or ignored and the basic assumption is that the pixels contain the information in the given image, and the goal of modeling is to cast a mathematical outlook onto this information. For example, image segmentation starts from the assumption that the gray level information in an image or volume resembles $\mathrm{N}$ classes (objects) that may or may not be contiguous or disjoint (overlapping or occluded) and the goal of segmentation would be to isolate the regions of similar nature into classes, and assign a color to each class differently from the rest. For example, a CCD image of an outdoor scene may contain water, grass, and dirt scattered elsewhere. Image segmentation would map that scene into three colors resembling water, grass and dirt; the water class, for example, may be in more than one location in the image spatial support, and may overlap in certain areas of the image with the dirt or grass regions.

From the above discussion it is obvious then that image modeling may take various view points, and as a result the algorithms vary. This field is extremely exciting and refreshing and is quite dominant in the image and signal analysis literature with domains like remote sensing, optical imaging, and biomedical imaging which have their own outlook and focus. Without going through the formidable task of surveying the methodologies and algorithms employed in image modeling - which is a daunting task and indeed out of the scope of this thesis - this work only projects a top view understanding of image modeling in CT imaging, the focus of this thesis, and highlights 
both the image formation standpoint and the algorithmic standpoint of image modeling. The image formation enables understanding the sources of uncertainty in CT imaging and enables proper analysis of such phenomenon as noise artifacts, motion artifacts, image blurring, etc. The algorithmic (goal-driven) aspect of image modeling enables descriptions of the information in the CT scan in order to: separate the lung tissue from the rest of the thorax, separate nodules from the anatomical structures in the lungs tissues, and help the ultimate goal of assigning a pathology for the detected and authenticated nodules.

To serve this understanding, this chapter is organized as follows. First the CT image formation is briefly described. Second, the sources of uncertainties in the CT scans are considered. As a test case, we consider the quality of the CT scans in the ELCAP clinical data to test the majority of the algorithms developed in this thesis. We show that the ELCAP data suffers from sensor noise and haziness (smearing effect). The modeling task here is reducing the noise and haziness effects. This thesis shows the results of employing two optimal filtering techniques: the Wiener Filter and the Anisotropic Diffusion filter. Hence, modeling here within is to address the errors/uncertainties in the CT scanning. After that we move into the intrinsic information in the CT scans in order to prepare for the important tasks of segmentation and nodule detection. Here, the focus is on examining the discriminatory features between lungs and thorax first, then between the lungs and nodules. In carrying out these two levels of classifications/discriminations we focus on understanding the intensity variation within regions (lungs and the rest of the thorax or nodules and anatomical structures of the lungs). In this process we consider two 
basic approaches: appearance (may also be called texture or intensity) models and spatial interaction (may also be called grouping) models [24]. Intensity may be modeled using a generalized form of the linear model. Spatial interaction may be modeled using the Markov model (e.g., [25]-[27]).

In summary, vis-à-vis CT image modeling we consider two aspects: a) correction of noise and haziness - this is accomplished by optimal filtering; and b) modeling the probability density function, that characterizes the gray scale intensity distribution, in order to serve the purpose of isolating the lung tissues from the thorax - this is accomplished using the linear model with focus on the special case of Gaussian kernels in which the Expectation-Maximization (EM) is the vehicle to carryout the estimation process (e.g., [14][16]). In all the developments to follow we shall avoid immersing into the ocean of the scientific literature that deals with image modeling as this indeed would drift us into unchartered territories beyond the focus of this thesis. Indeed, the references listed in this chapter are no more than an arbitrary list that does not represent the original works on image modeling, rather those that we used and found accessible (e.g., [24-27]).

\section{B. CT Image Formation}

The fundamental problem of reconstruction from projections which describes CT slice reconstructions may be described as follows [21][22]: Given a set of 1-D projections and the angles at which these projections were taken. How does one reconstruct the 2-D image from which these projections were taken? For illustration purpose and simplicity of analysis we only consider the parallel beams case which considers x-rays hitting an 
object $\mathrm{g}(\mathrm{x}, \mathrm{y})$ in parallel and generating a projection $\mathrm{P}_{\boldsymbol{\theta}}(\mathrm{t})$ per angle $\boldsymbol{\theta}$, as shown in Figure 6.

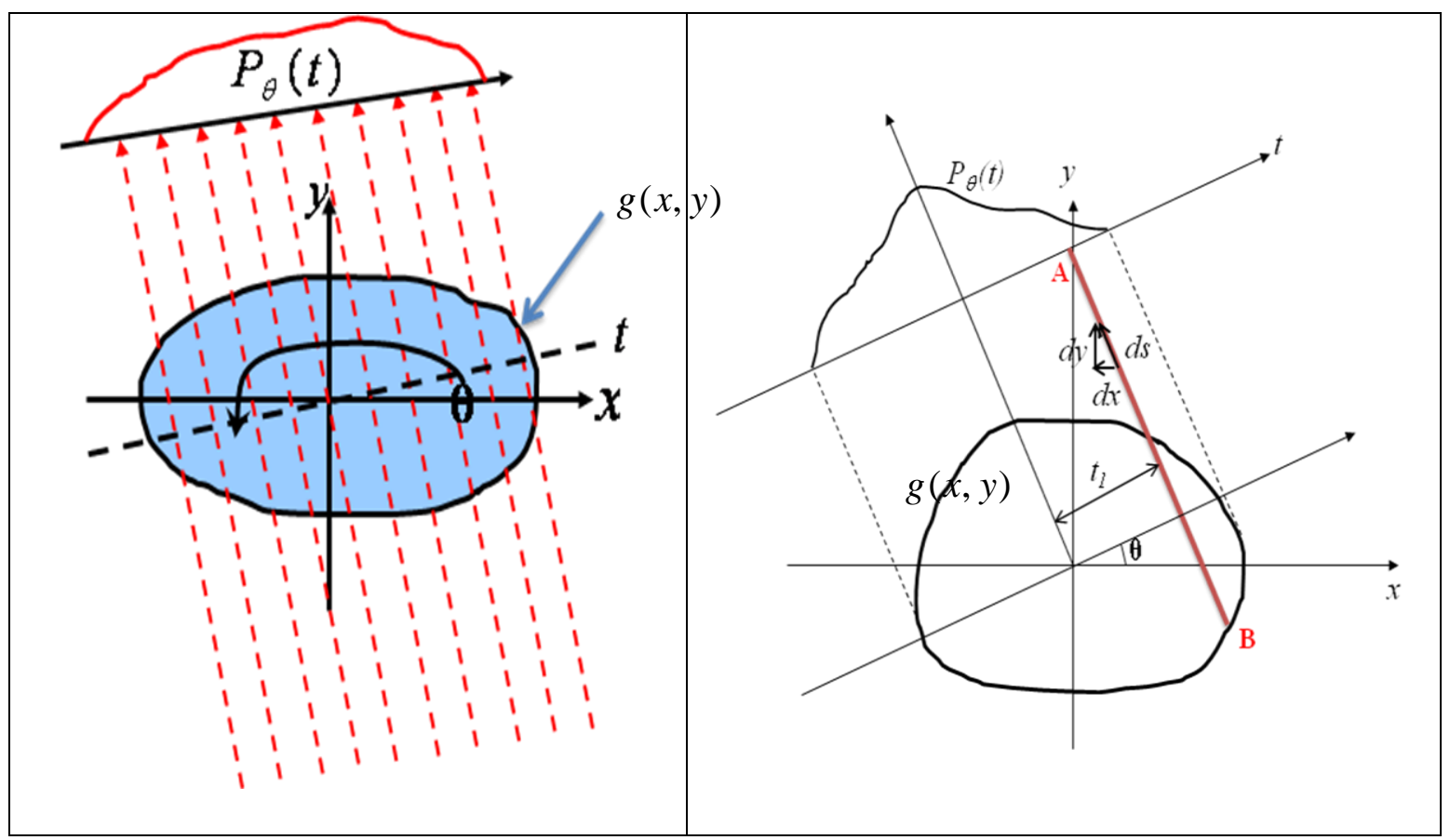

Figure 6: Illustration of parallel beam projections where a set of rays hit an object $g(x, y)$ at an angle $P_{\boldsymbol{\theta}}(t)$ per angle $\boldsymbol{\theta}$. On the right we highlight the parameters relating one ray $(A B)$ to the projection process.

From basic principles, we can show that the relationship between the projection $\mathrm{P}_{\boldsymbol{\theta}}(\mathrm{t})$ and the original function (object) $\mathrm{g}(\mathrm{x}, \mathrm{y})$ is given by the Radon Transform [21].

$$
\begin{aligned}
P_{\theta}(t) & =\int_{-\infty}^{\infty} \int_{-\infty}^{\infty} g|x, y| \delta|x \cos \theta+y \sin \theta-t| d x d y \\
& =\int_{-\infty}^{\infty} g(t, s) d s .
\end{aligned}
$$

where $\delta($.$) is an impulse sheath defined on the ray x \cos \theta+y \sin \theta \equiv t$ 
In the frequency domain, the above equation can be written as:

$$
\begin{gathered}
\left.S_{\theta}(f)=\mathfrak{I}_{1 D}\left|P_{\theta}\right| t \mid\right\}=\int_{-\infty}^{\infty} P_{\theta}|t| e^{-j 2 \pi f t} d t \\
g|x, y|=\int_{0}^{\pi} \underbrace{[\int_{-\infty}^{\infty} \overbrace{}^{\text {Filter Response }} S_{\theta}|f| e^{j 2 \pi f t} d f]}_{Q_{\theta}(t)} d \theta \\
t=x \cos \theta+y \sin \theta
\end{gathered}
$$

Therefore, we can relate the function $\mathrm{g}(\mathrm{x}, \mathrm{y})$ with respect to the Fourier transform of the projections $\mathrm{P}_{\boldsymbol{\theta}}(\mathrm{t})$ for all angles $\theta \in|0, \pi|$.

Numerical implementations of Equation (2.3) relates the quality of the estimated $\mathrm{g}(\mathrm{x}, \mathrm{y})$ with respect to the number of projections (sampling with respect to $\boldsymbol{\theta})$ and the number of rays (sampling with respect to the parameter $t$ ). Figure 7 shows the effect of reconstruction of one projection of a scientific phantom. 
Space Domain

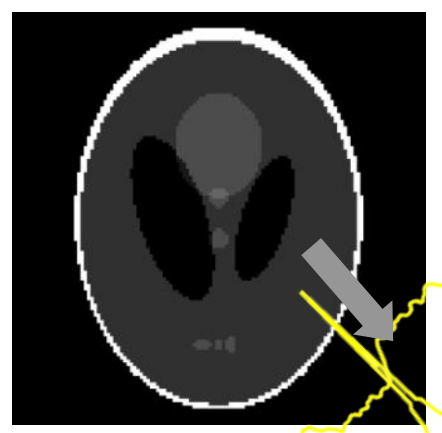

Frequency Domain $S(f, \theta)$

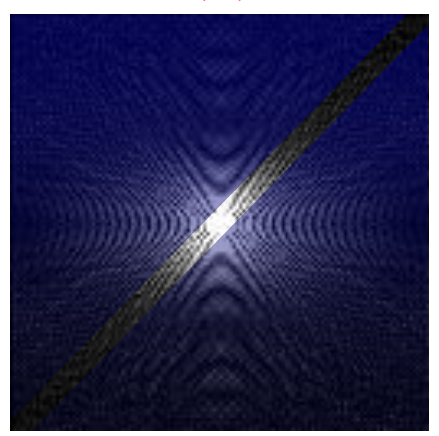

Figure 7: Illustration of reconstruction from one projection of the Shepp-Logan model [29] implemented using MatLab. The inverse Fourier transform provides the estimate of $g(x, y)$.

The above illustrations are provided here just to highlight the main issues involved in reconstruction from projections.

Image quality, in general, depends on the particular imaging modality used. For each modality, the range of image quality may be considerable, depending on the characteristics and set up of the particular medical imaging system. Following six important factors effect image quality:

- Contrast: High contrast allows easier identification of individual objects.

- Resolution: The ability of an imaging system to depict details. 
- Noise: An image may be corrupted by random fluctuations in image intensity.

- Artifacts: Obscure important features, or falsely interpreted as abnormal findings.

- Distortion: Changes of shape, size, position, and other geometric characteristics.

- Accuracy: The quality of medical images should be judged.

With respect to modern CT scanners, the following issues determine the quality of the scan:

- Number of rays (detectors): The number of rays over the same field-ofview (FOV) has a strong effect on the spatial resolution and quality.

- Number of projections per scan: The more number of projections, the better quality of scan.

- Slice thickness (will affect the resolution of the image): Large slice thickness reduces spatial resolution.

- Pitch (spacing between slices): The greater pitch, the less spatial resolution.

Extraneous effects on the scanning include subject motion (generates blurring effect), scanner condition (heating due to overuse may lead to haziness). Figure 8 shows CT slices of high resolution and low resolution scans. Of course, the issues with respect to 
lung cancer are mainly: cost (as periodic scanning may be recommended), radiation hazards, and quality of scanning. These are the main issues that must be thoroughly examined (hopefully will be answered by the NLST study, and may in fact be answered at least in part from the analysis of the Louisville scanning study).
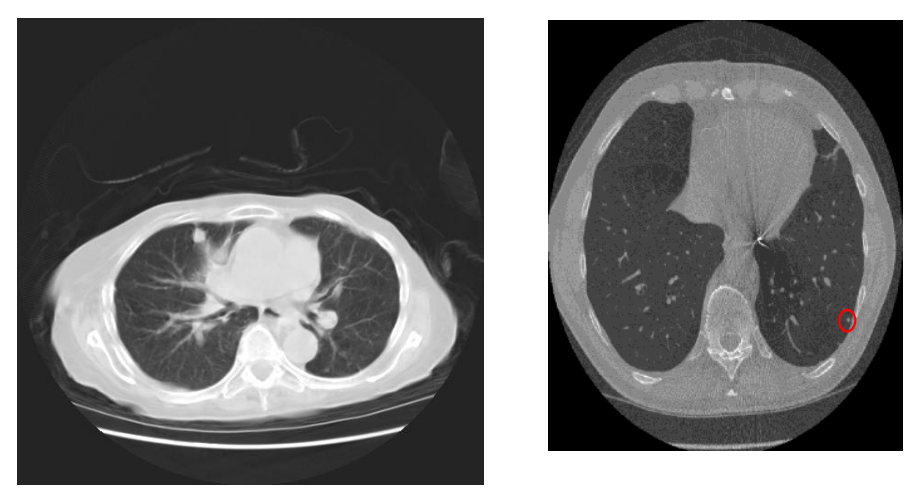

Figure 8: Examples of high (left) and low (right) resolution thoracic scans.

\section{Modeling the Uncertainty}

Sources of uncertainty vary from one imaging modality to another; hence, the modeling to improve a scan varies in CT and MRI (see [22][23] for an example). These models forget the image formation process and works at the output; i.e., given a scan, how to make it better? Better may include noise removal, enhancing the contrast, removal of blurring/haziness effects, etc. This type of modeling is studied in the image processing and analysis literature under various names including "enhancement', "restoration", and "recovery". 
As indicated before, the ELCAP data which is the focus of clinical evaluation suffers from noise and haziness effects. We shall model these two effects and use some wellestablished optimal filtering approaches to remedy them.

\section{Additive Noise (Weiner Filtering)}

The basic Weiner filter model in the discrete-time domain (numeric domain) is as follows: the observed CT slice $\mathrm{g}(\mathrm{m}, \mathrm{n})$ is considered to result from disturbing an original (perfect image) $s(m, n)$ corrupted with a noise $\eta(m, n)[28]$. The quantities $g(),. s($.$) and$ $\eta($.$) are assumed to be sample functions from a random field g(),. s($.$) and \eta($.$) defined on$ the same spatial support (e.g., the rows and columns of a discrete image). It is assumed that the statistics of these random fields are known, in particular the power spectral density functions, $P_{s}\left(f_{1}, f_{2}\right), P_{\eta}\left(f_{1}, f_{2}\right)$, and $P_{g}\left(f_{1}, f_{2}\right)$ are assumed to be known; where $f_{1}$ and $f_{2}$ are continuous, have units of cycles, and defined over the interval $[0,1]$. The goal is to design an operator that works on $g(m, n)$ and provide an estimate for $s(m, n)$ that corrects for the noise. The Wiener filter is an operator $h(m, n)$ that generates the desired estimate $f($.$) (should be added) such that the mean square error between the processes f($. and $s($.$) is minimum; i.e., the goal is to find \mathrm{h}(\mathrm{m}, \mathrm{n})$ such that

$$
E\left(|f(m, n)-s(m, n)|^{2}\right) \text { is minimum }
$$

The model is illustrated in Figure 9. 


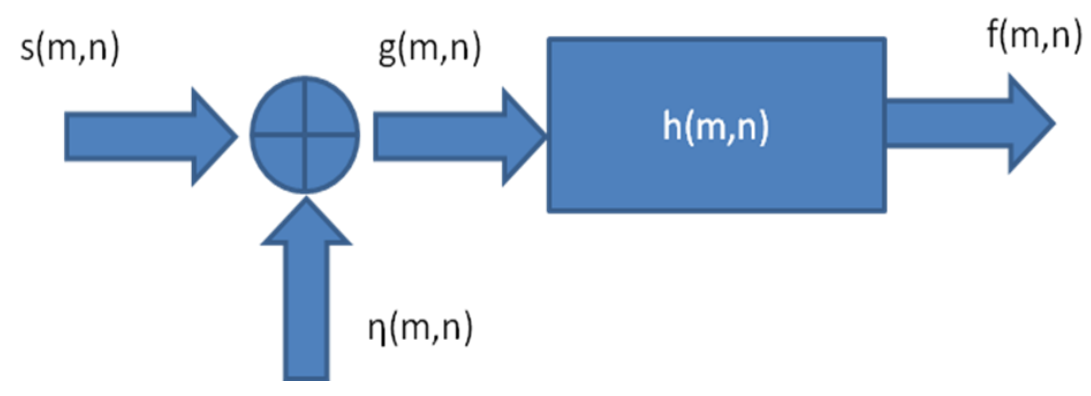

Figure 9: Modeling the observed image as an ideal image $s(m, n)$ disturbed with an additive noise $\eta(m, n)$. The Weiner filter is an operator $h(m, n)$ that provides an estimate for $s(m, n)$ in the mean square error sense.

With the simplifying assumption that the processes $\mathrm{s}($.$) and \eta($.$) are uncorrelated, we can$ easily show that the Weiner filter equation is as follows (e.g., [28]):

$$
H\left(f_{1}, f_{2}\right)=\frac{P_{s}\left(f_{1}, f_{2}\right)}{P_{s}\left(f_{1}, f_{2}\right)+P_{\eta}\left(f_{1}, f_{2}\right)}
$$

The Weiner filter is linear and shift-invariant, hence, the output $f(m, n)$ is obtained from the convolution of the observed image $g(m, n)$ and the filter $h(m, n)$, which is obtained from the inverse Fourier transform of Equation (2.5). The implementation of the Weiner filtering operator requires the knowledge of the noise and observed image statistics. Below we describe how these quantities were estimated from the CT scans of the ELCAP data.

Figure 10 shows a sample of 16 slices from one patient of the ELCAP data. As can be observed, the slices suffer from noise (which is considered additive) and haziness (blurring) effects. The corresponding histograms, Figure 11, are distinctly bi-modal. 


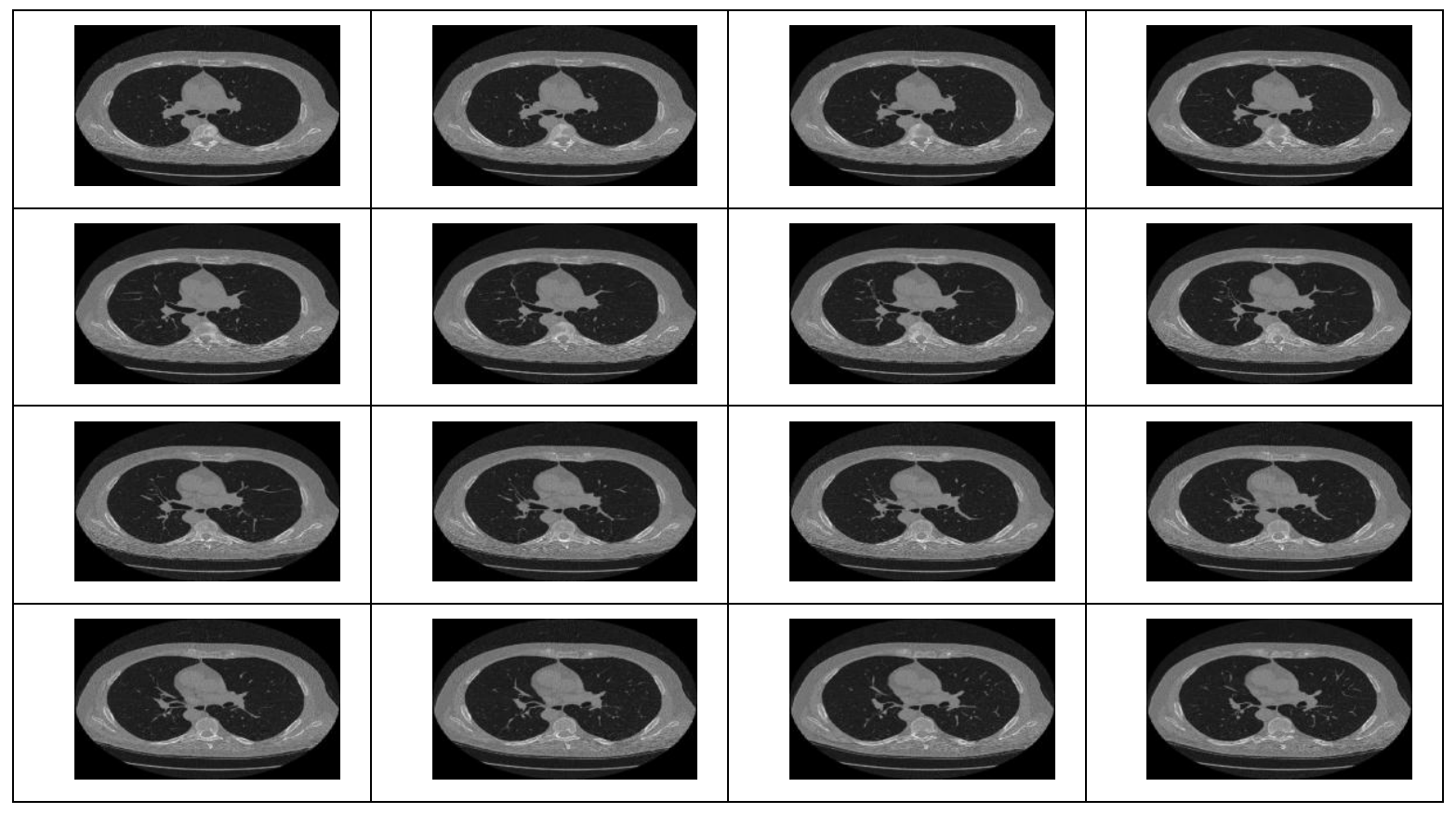

Figure 10: A sample of 16 slices from a patient scan of the ELCAP study.

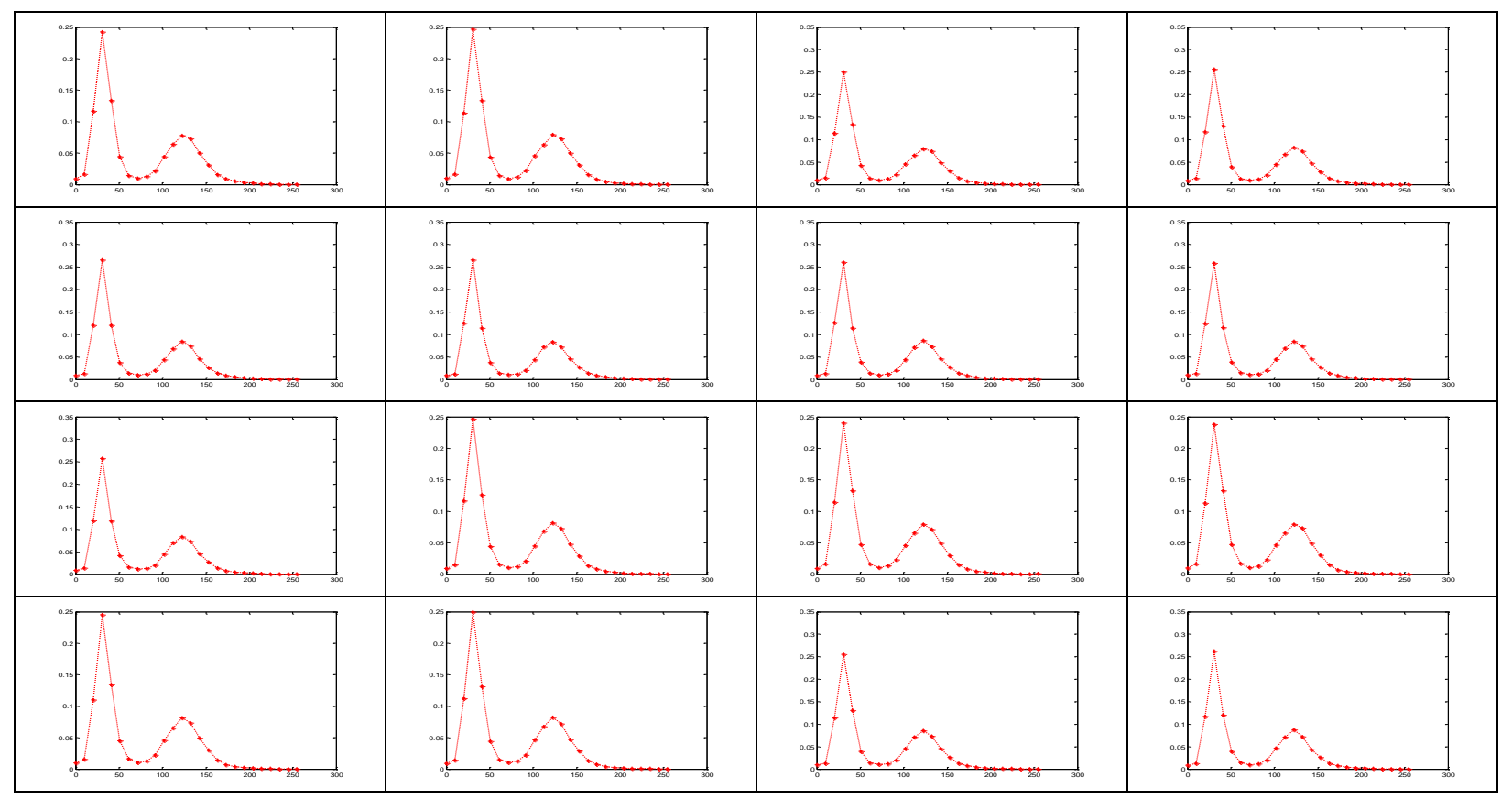

Figure 11: Histogram of the slices in Figure 10. Note the distinct bimodal nature of the histograms. 
The noise histogram can be estimated by manually cropping the thorax region from the scan (i.e., we isolated the background from the chest data).

(l)

Figure 12: Cropped noise background of the 16 slices in Figure 10. For Visualization purposes the images are placed on a white background. 


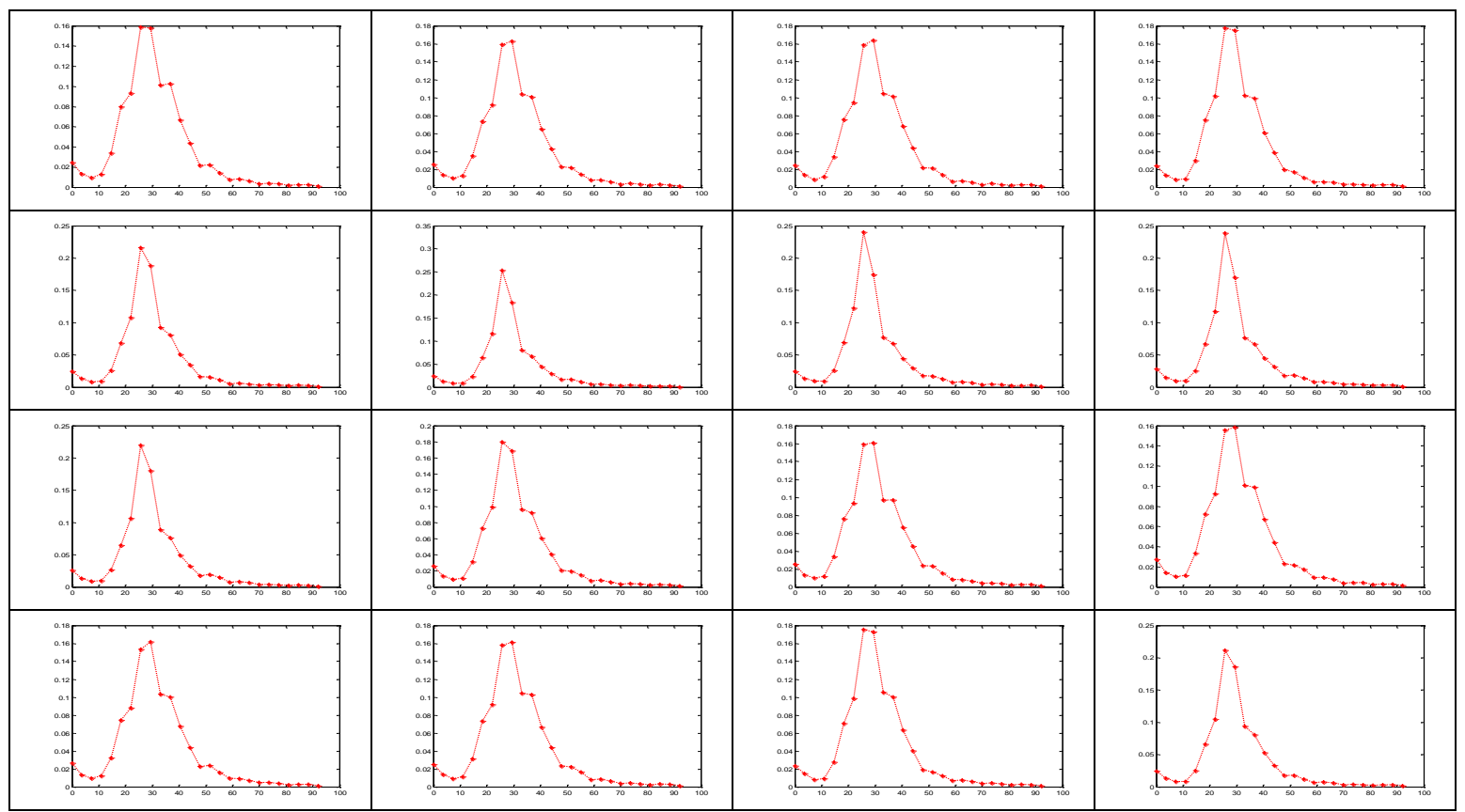

Figure 13: The histogram of the noise from the cropping operation.

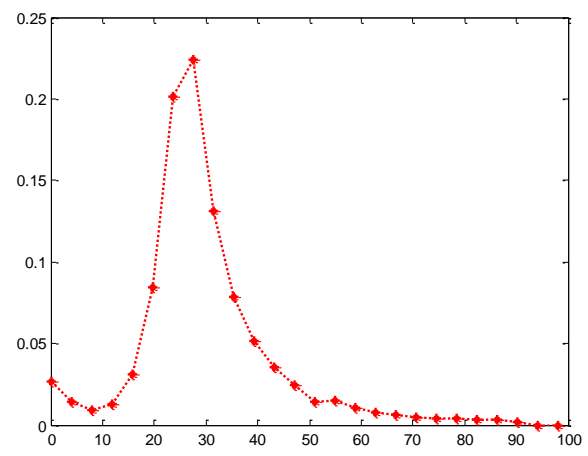

Figure 14: Average noise histogram obtained from 16 slices (in Figure 10).

Indeed, performing the cropping operation in Figure 10 over 16 full scans (each having 235-280 slices) of the ELCAP data and averaging all their histograms per scan is shown in Figure 15, and the overall average (of the 16 scans histograms of the slices) is shown in Figure 16. 
It is obvious that the histogram is primarily uni-modal and can be adequately modeled by a single Gaussian kernel.

The parameters of the Gaussian distribution may be estimated using the least square estimation (LSE) or the maximum likelihood estimation (MLE) approaches which are equivalent for the Gaussian case.

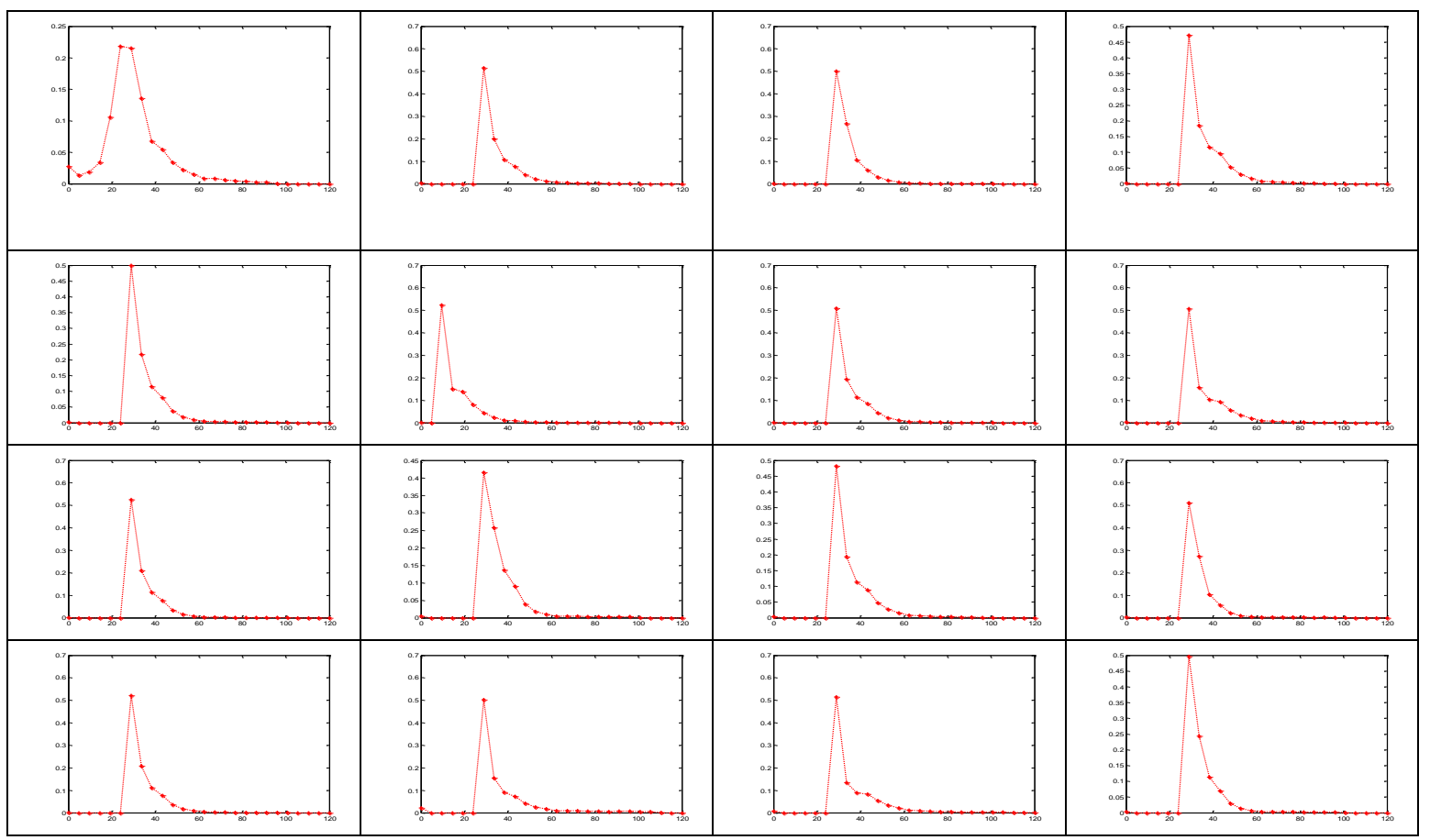

Figure 15: The histogram of the noise from the cropping operation resulting from 16 full scans of the ELCAP data. Each histogram is the average of all the histograms corresponding to cropping the noise off all the slices in the 16 scans. 


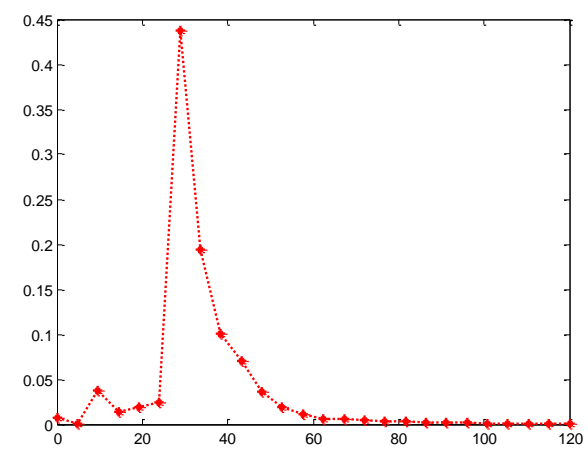

Figure 16: Average noise histogram obtained from 16 scans (in Figure 15).

The maximum likelihood estimation of mean and variance of the noise histogram has the following form:

$$
\begin{gathered}
\mu_{n}=\frac{1}{N} \sum \sum \eta(m, n) \\
\sigma_{n}^{2}=\frac{1}{N} \sum \sum[\eta(m, n)-\mu]^{2}
\end{gathered}
$$

where $\mathrm{N}$ is the total number of samples in cropped regions. These values may also be estimated from the histogram of an ensemble as in Figure 15 or Figure 16. Indeed, if we fit a single Gaussian to the noise histogram in Figure 16 using the values in Equation 2.6 and 2.7 (or using the EM algorithm, which will be discussed later) we can see that the mean value is around 33 and the standard deviation is about 3 . Hence, a smoothing filter of width $\pm 3 \sigma_{\eta}$ may be adequate for removing the noise. As we shall see, an adaptive implementation of the Weiner filter on blocks of size $9 \times 9$ or $11 \times 11$ provides adequate noise removal. Likewise, we may use a Gaussian filter with similar width to remove the noise.

We can calculate the power spectral density function using numerical estimation approaches such as the periodogram (e.g., [28]). 
A simplifying example may be made with respect to the noise, in which we may consider the noise to be white Gaussian; therefore, the power spectrum density is constant overall frequency ranges with value of ${\sigma_{\eta}}^{2}$.

The power spectrum of the ideal image $s(m, n)$ is obtained by subtracting the effect of the noise from the observed image $\mathrm{g}(\mathrm{m}, \mathrm{n})$ and using the peridogram approach as well.

In implementing the Weiner filter, a local approach is used; i.e., the statistics are evaluated on blocks (regions) of the image instead of the entire image. These blocks may be overlapping or non-overlapping. This is known as the adaptive Weiner filter (e.g., Lim, 1990) [28]. Adaptive Weiner filtering enhances the noise removal more than the global application of the filter. These improvements results from using the local statistics of the pixels within a neighborhood instead of the entire spatial support. This is to be expected as the degree of dependence of pixels on neighboring ones decreases with distance.

Assuming an additive white noise the power spectral density of the noise $\eta(m, n)$ is:

$$
\mathrm{P}_{\eta}\left(\mathrm{f}_{1}, \mathrm{f}_{2}\right)=\sigma_{\eta}{ }^{2}
$$

On a small neighborhood of the image, we may assume that the process $s(\mathrm{~m}, \mathrm{n})$ stationary (i.e., its statistics do not change by small shifts), we can model the ideal image $\mathrm{s}(\mathrm{m}, \mathrm{n})$ as:

$$
\mathrm{s}(\mathrm{m}, \mathrm{n})=\mu_{\mathrm{s}}+\sigma_{\mathrm{s}} \mathrm{w}(\mathrm{m}, \mathrm{n})
$$


where $\mu_{\mathrm{s}}$ and $\sigma_{\mathrm{s}}$ are the local mean and standard deviations of $\mathrm{s}(\mathrm{m}, \mathrm{n})$, and $\mathrm{w}(\mathrm{m}, \mathrm{n})$ is zero-mean white noise with unit variance. Therefore, the power spectral density of the image process $P_{s}\left(f_{1}, f_{2}\right)$ is given by the following equation:

$$
P_{s}\left(f_{1}, f_{2}\right)=\sigma_{s}^{2}
$$

Hence, the adaptive Weiner filter becomes:

$$
H\left(f_{1}, f_{2}\right)=\frac{\sigma_{s}^{2}}{\sigma_{s}^{2}+\sigma_{\eta}^{2}}
$$

Taking the inverse Fourier Transform, we obtain

$$
h(m, n)=\left[\frac{\sigma_{s}^{2}}{\sigma_{s}^{2}+\sigma_{\eta}^{2}}\right] \delta(m, n)
$$

where $\delta(m, n)$ is the Kroncker-delta function, which is a unit value with $\mathrm{m}=\mathrm{n}$ and is zero elsewhere. The recovered image $f(m, n)$ in Figure 9 would be:

$$
\begin{aligned}
f(m, n) & =g(m, n) * h(m, n)=(s(m, n)+\eta(m, n)) * h(m, n)=\mu_{s}+\left(g(m, n)-\mu_{s}\right) * h(m, n) \\
& =\mu_{s}+\left[\frac{\sigma_{s}^{2}}{\sigma_{s}^{2}+\sigma_{\eta}^{2}}\right]\left(g(m, n)-\mu_{s}\right)
\end{aligned}
$$

As a window operation, the adaptive Weiner filter equation (2.13) becomes:

$$
f(m, n)=\mu_{s}(m, n)+\left[\frac{\sigma_{s}^{2}(m, n)}{\sigma_{s}^{2}(m, n)+\sigma_{\eta}^{2}(m, n)}\right]\left(g(m, n)-\mu_{s}(m, n)\right)
$$

where the quantities $\mu_{\mathrm{s}}(\mathrm{m}, \mathrm{n})$ and $\sigma_{\mathrm{s}}{ }^{2}(\mathrm{~m}, \mathrm{n})$ are calculated on neighborhoods (blocks) centered at the current pixel location $(\mathrm{m}, \mathrm{n})$ [28]. The following estimates are used: 


$$
\mu_{s}(m, n)=\frac{1}{(2 M+1)^{2}} \sum \sum g\left(k_{1}, k_{2}\right)
$$

Since $g(m, n)=s(m, n)+\eta(m, n)$, and we assume $s(m, n)$ and $\eta(m, n)$ to be uncorrelated, then

$$
\sigma_{g}^{2}=\sigma_{s}^{2}+\sigma_{\eta}^{2}
$$

where $\sigma_{\mathrm{g}}^{2}$ may be estimated from the observed image $\mathrm{g}(\mathrm{m}, \mathrm{n})$ as follows:

$$
\sigma_{g}^{2}=\frac{1}{(2 M+1)^{2}} \sum \sum\left(g\left(k_{1}, k_{2}\right)-\mu_{s}(m, n)\right)^{2}
$$

To sum up, the adaptive Weiner filter is implemented as follows (Lim, 1990 [28]):

\section{Algorithm:}

Step 1: estimate $\mu_{\eta}$ and $\sigma_{\eta}^{2}$ from Equation 2.6 and 2.7

Step 2: estimate $\mu_{\mathrm{s}}(\mathrm{m}, \mathrm{n})$ and $\sigma_{\mathrm{s}}^{2}$ from Equations (2.15), (2.16) and (2.17)

Step 3: estimate the Weiner filter function $\mathrm{h}(\mathrm{m}, \mathrm{n})$ from Equation 2.12, or equivalently, evaluate the smoothed signal $\mathrm{f}(\mathrm{m}, \mathrm{n})$ using Equation (2.14).

In implementing the above equations, we used a built in Matlab function (Wiener2).

Figure 17 shows the results of adaptive Weiner filtering with various block sizes applied to slices from the ELCAP study with known nodules.

We conducted the Weiner filtering with higher block size, finding the blurring effect dramatically increased with block size above $9 \times 9$. Since our goal is to maintain the nodules while reducing the additive noise effect, our extensive evaluation on the ELCAP dataset suggests that a block of sizes $7 \times 7$ or 9x9 are adequate. The adaptive Weiner filter is quite fast in implementation. 


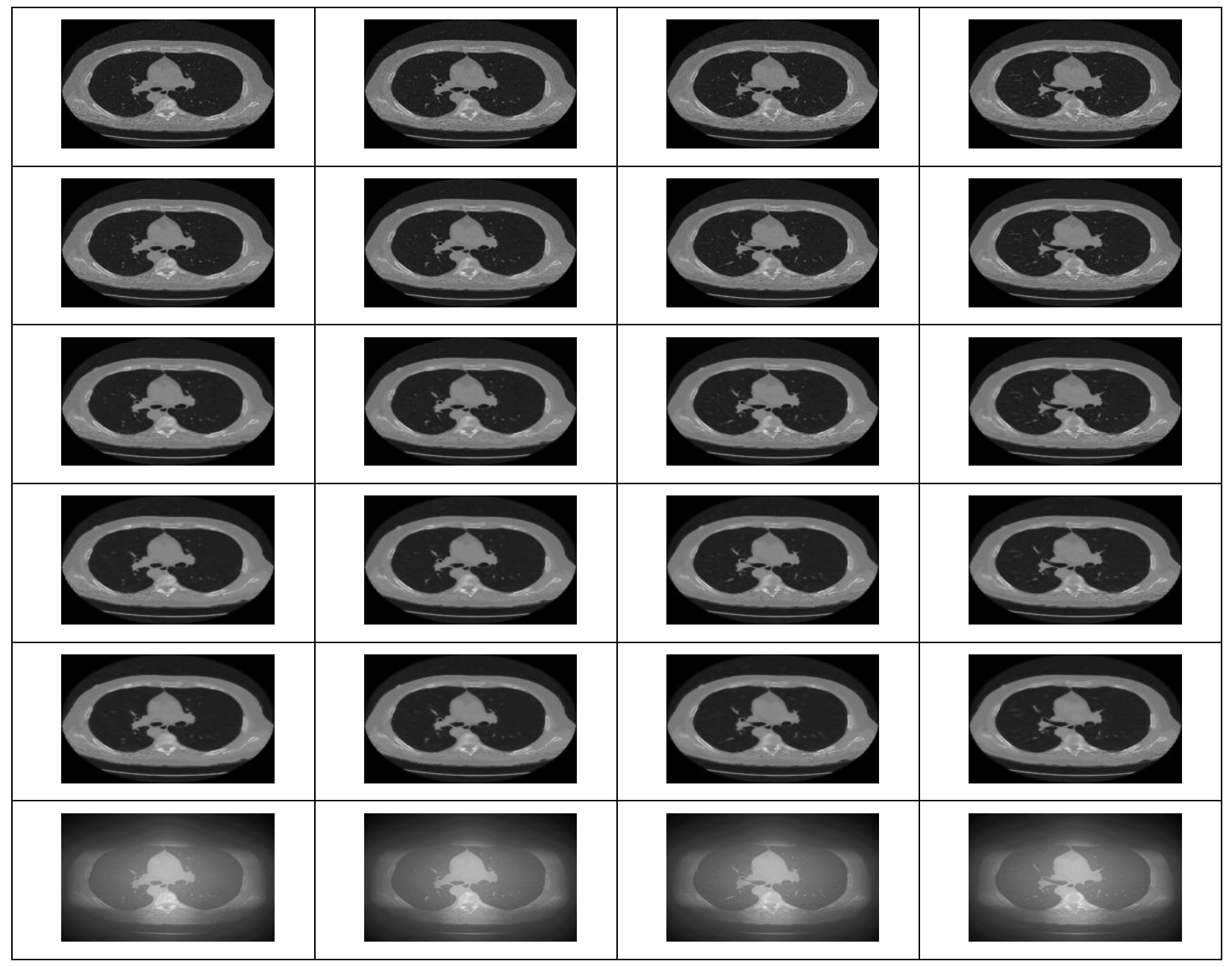

Figure 17: Wiener filter applied onto four original images with different window sizes. The first row represents the original images, second row is the wiener filter applied with window [3 3], third row is with window [7 7], fourth row is with window [9 9], fifth row is with window [11 11] and the last row is window size [511 511].

In general, the Weiner filter reduced the noise somewhat, but also induced a blurring effect in the images. In our application, the desire for image quality, focus, and clarity is important, but most important is no loss of pixel information due to filtering. In our application, the lung nodules are the desired information. Below, we discuss another popular filtering technique, the anisotropic diffusion filter that is commonly used in 
medical imaging analysis. This filter is shown to provide a better performance than the Weiner filter in terms of noise smoothing and maintaining the nodules.

\section{Anisotropic Diffusion Filtering [29-30]}

Anisotropic diffusion filtering is variational approach of filtering which reduces image noise while maintaining edge information. The derivation is involved and is beyond the focus of this thesis. It suffices for our purpose that it is a well proven approach in various image analysis applications. We have used a Matlab implementation of the technique and have compared it with the Weiner filtering approach, which we detailed above.

The following requirements should ideally be fulfilled:

a) Minimize information loss by preserving object boundaries and detailed structures,

b) Efficiently remove noise in regions of homogeneous physical properties,

c) Enhance morphological definition by sharpening discontinuities.

Smoothness is formulated as a diffusive process:

$$
\frac{\partial}{\partial t} u(X, t)=\operatorname{div}(c(X, t) \nabla u(X, t))
$$

where, $X=(x, y), u(X, t)$ is the diffusion strength, and div is the divergence operator. $C(X, t)$ is the diffusion function, which could be chosen from the following,

$$
c_{1}(X, t)=\exp \left(-\left(\frac{\mid \nabla I(X, t \mid)}{k}\right)\right)
$$




$$
c_{2}(X, t)=\frac{1}{1+\left(\frac{\mid \nabla I(X, t \mid)}{k}\right)}
$$

where, the parameter $k$ is chosen to control the noise level and the edge strength.

$$
\begin{aligned}
& \frac{\partial}{\partial t} I(X, t)=\operatorname{div}(c(X, t) * \operatorname{grad} I(X, t))=\nabla^{T}(c(X, t) * \nabla I(X, t)) \\
& =\frac{\partial}{\partial x}\left(c(X, t) * \frac{\partial}{\partial x} I(X, t)\right)+\frac{\partial}{\partial y}\left(c(X, t) * \frac{\partial}{\partial x} I(X, t)\right) \\
& =\frac{1}{\Delta x^{2}}\left(c\left(x+\frac{\Delta x}{2}, y, t\right) *(I(x+\Delta x, y, t)-I(x, y, t))-c\left(x-\frac{\Delta x}{2}, y, t\right) *(I(x, y, t)-I(x-\Delta x, y, t))\right) \\
& +\frac{1}{\Delta y^{2}}\left(c\left(x, y+\frac{\Delta y}{2}, t\right) *(I(x, y+\Delta y, t)-I(x, y, t))-c\left(x, y-\frac{\Delta y}{2}, t\right) *(I(x, y, t)-I(x, y-\Delta y, t))\right) \\
& =\phi_{\text {east }}-\phi_{\text {west }}+\phi_{\text {north }}-\phi_{\text {south }}
\end{aligned}
$$

where, $\nabla$ is the divergence operator.

Under 2D case, the pixel intensities are updated by the local sum of the flow contributions:

$$
T(t+\Delta t) \approx I(t)+\Delta t \times \frac{\partial}{\partial t} I(t)=I(t)+\Delta t \times\left(\phi_{\text {east }}-\phi_{\text {west }}+\phi_{\text {north }}-\phi_{\text {south }}\right)
$$

\section{Algorithm: Anisotropic Diffusion Filtering [30]}

1) Load in the original image;

2) Choose the integration constant (time interval) $\Delta$ t based on the different neighborhood structures, e.g. for $2 \mathrm{D}$ case, 4 neighboring pixels, $0<\Delta \mathrm{t}<1 / 5$.

3) Determine the diffusion function kernels by choosing either Equation (2.19) or (2.20);

4) Follow Equation (2.21) to compute the $\Phi_{\text {east }}, \Phi_{\text {west }} \Phi_{\text {north, }}$ and $\Phi_{\text {south, }}$, then

5) Follow Equation (2.22) to update the new value at each pixel value;

6) Iteration stops if the value difference at each pixel during two consecutive iterations is less than 0.5 . 
Below we show the performance of anisotropic diffusion filtering on CT imaging.

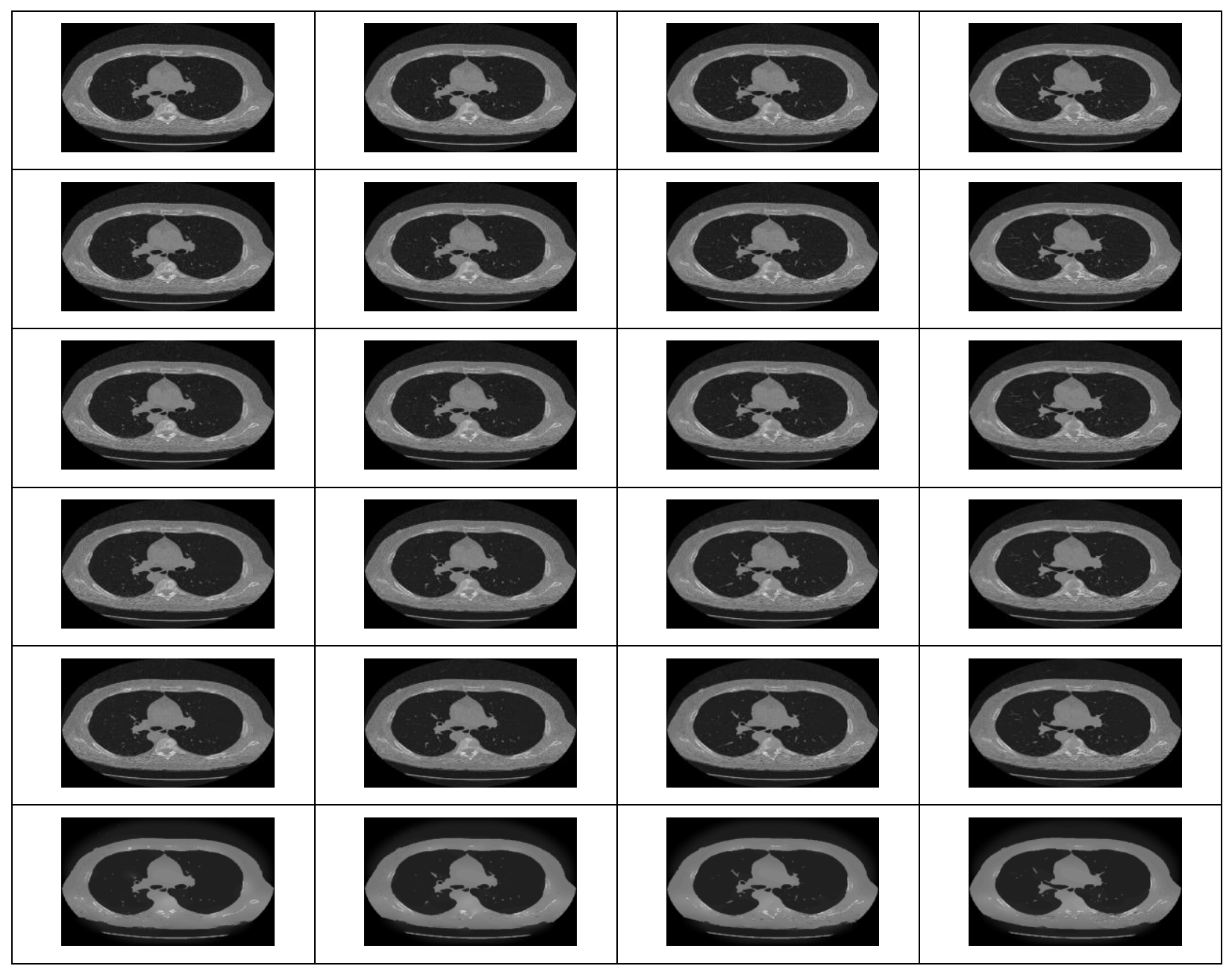

Figure 18: Anisotropic filter for different kappa values. The first row are the original images for four original images, second row is the filtered image with $k=($ standard deviation of intensity - std - values in a slice $) / 511$, third row $k=($ std of slice $) / 21$, fourth $k=($ std of slice $) / 11$, fifth row $k=($ std of slice $) / 9$ and the last row $k=($ std of slice $) / 3$. 


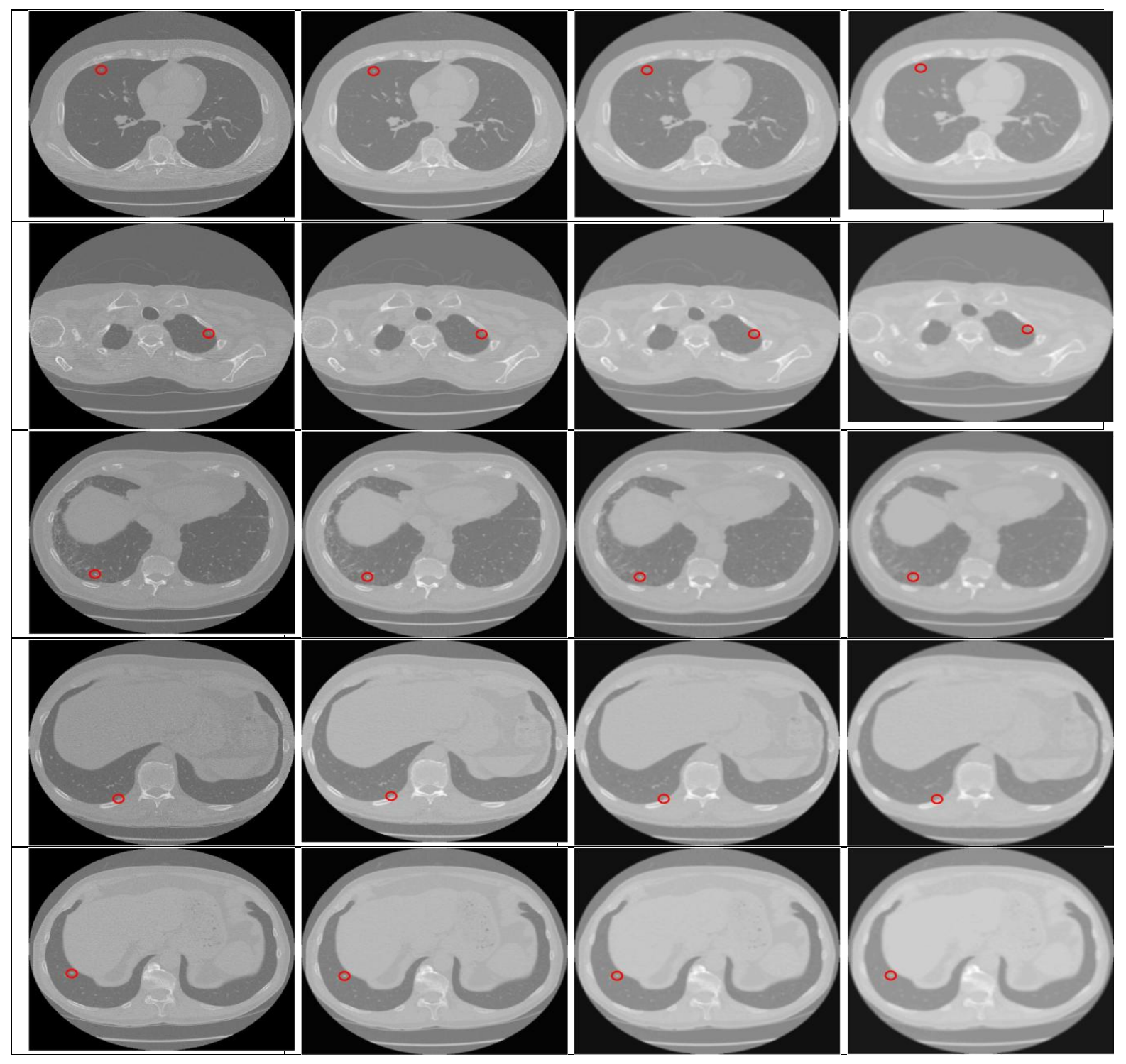

Figure 19: Performance of the adaptive Weiner filter on data sets of known nodule locations. From left to right: original slices, adaptive Weiner filtering of size 3x3, 5x5 and $7 x 7$. (Red circle shows the nodule location) 


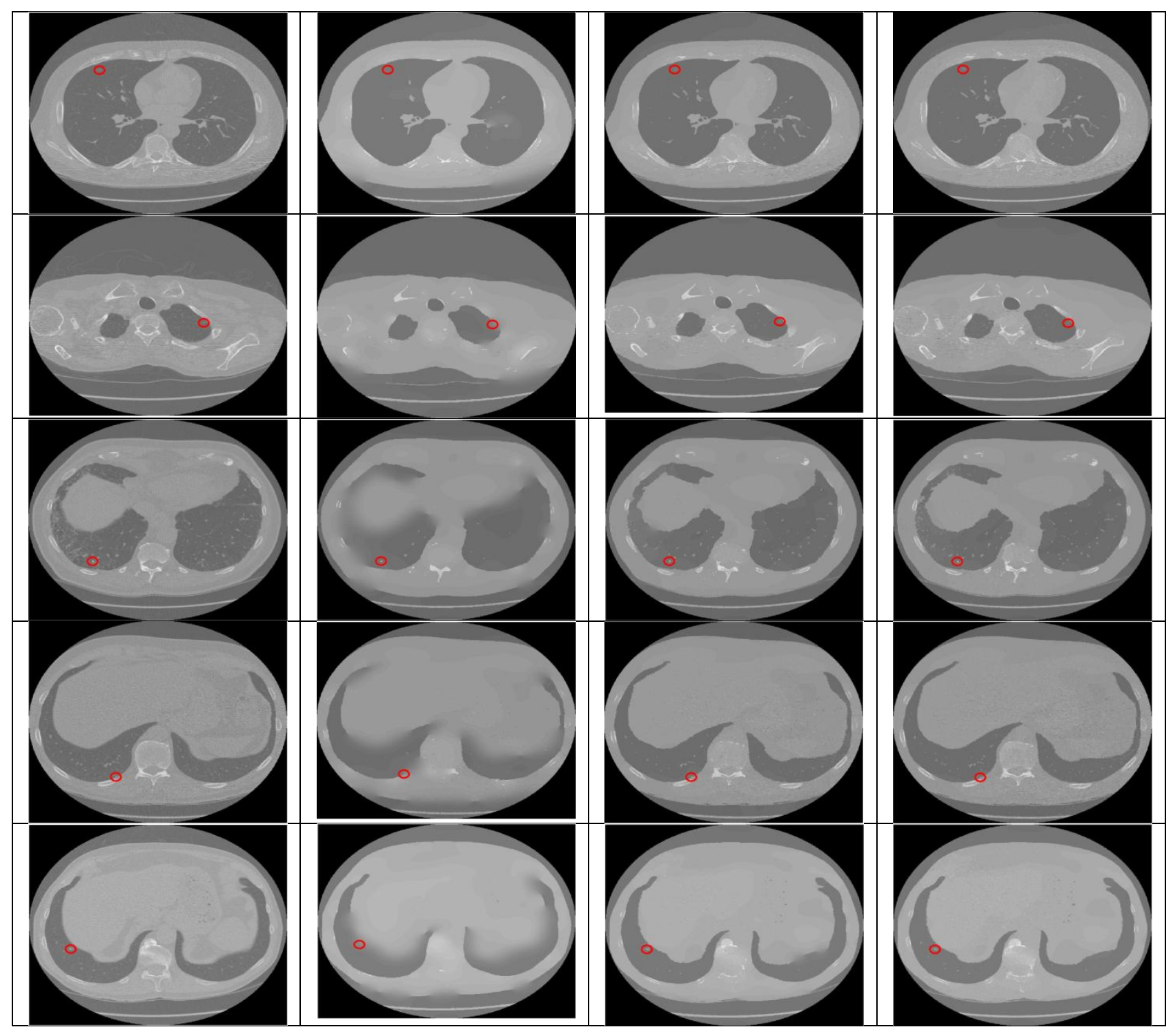

Figure 20: Performance of the anisotropic diffusion filter on data sets of known nodule locations. From left to right: original slices, anisotropic diffusion filtering of three different $k$ parameters $(k=$ standard deviation $/ N ; N=5,8,10$ respectively). (Red circle shows the nodule location) 


\section{Performance Evaluation}

We tested the performance of the filtering operation in terms of two measures: a) noise removal; b) maintaining the important information; in our case the nodules. We are most concerned about the loss of nodules due to the filtering step. Filtering may lead to removal of the nodules or altering their locations, which may induce another uncertainty with respect to the anatomy of the lung tissues versus the lung nodules.

To have a measure of the information loss due to filtering, we applied the Weiner and anisotropic filters on sample slices, which have pre-identified nodules. We measured visibly - the retaining or the missing of nodules in the filter output.

From these results and observations on filtering, it can be concluded that an isotropic filtering or an adaptive Weiner filtering on $7 \times 7$ or $9 \times 9$ blocks have the same performance. In the subsequent analysis, we use anisotropic diffusion filter.

\section{Modeling the Intensity}

In image analysis, often density estimation is based on modeling the histogram. The problem may be stated as follows: Let $\mathrm{q}=\{0, \ldots, \mathrm{Q}-1\}$ and $\mathrm{k}=\{1, \ldots, \mathrm{K}\}$ denote sets of gray levels q and region labels $\mathrm{k}$, respectively. Here, Q is the number of gray levels and $\mathrm{K}$ is the number of image modes, i.e. peaks in the gray level frequency distribution, e.g., for a bimodal image, $\mathrm{K}=2$. The analysis based on the assumption that each dominant image mode corresponds to a particular class of objects to be found in the image. Let $S=\{(i, j): 1 \leq i \leq I, 1 \leq j \leq J\}$ be a finite arithmetic grid supporting gray level 
images $Y: S \rightarrow Q$ and their region maps $\mathrm{X}: \mathrm{S} \rightarrow \mathrm{K}$. Let $f(q): q \rightarrow Q$ be an empirical relative frequency distribution of gray levels q collected over the whole image Y. In summary, assuming independent signals in the pixels $(i, j) \in S$, given $f(q)$, the issue is how to estimate in an unsupervised manner; (1) The mixed density distrubution that represents $f(q)$, and (2) The marginal density for each class.

The classical linear combinations of Gaussian (LCG) is based on combinations of Gaussian kernels. In this thesis, we refer to the classical linear model with Gaussian kernels as LCG. Of course, other kernels may be used (and have been used in literature) other than the Gaussian but is not considered in this paper.

Noting that all the histograms of the lung CT data are strictly bi-modal (see Figure 11), we have an easier case of fitting the histogarm which can be obatined by using a linear model with Gaussian kernels. To estimate the parameters of the model shown, the Expectation Maximization (EM) algorithm (e.g, [31-34]) has been used. We discuss below how the estimation can be performed using the EM algorithm.

\section{The E-M Algorithm}

The Expectation-Maximization (EM) algorithm is an iterative algorithm that finds the parameters which maximize the log-likelihood when there are missing data, in our case here, the membership of a data point $x_{i}$ to a certain class/component $z_{i}$ or the probability of membership to a certain class/component. Therefore we can view the complete data as the combination of the missing data and the observed data, for the case of Gaussian mixture, the complete data consist of pairs $\left(x_{i}, z_{i}\right)$ and $i=1,2, \ldots, n$. 
Let $X$ and $Z$ denote the vector of all observed data $\left\{x_{1}, x_{2}, \ldots, x_{n}\right\}$ and hidden data $\left\{z_{1}, z_{2}, \ldots z_{n}\right\}$ respectively. Let $\theta^{t}$ be the parameter estimate at the $t$-th iteration. Define the Q-function of $\theta$ as the expected value of the complete data log-likelihood given the observed data $X$ and the current component parameters $\theta^{t}$.

$$
\begin{aligned}
Q\left(\theta ; \theta^{t}\right) & \left.=E_{Z} \mid \operatorname{pg} p \ll, Z ; \theta\right] X, \theta^{t}{ }_{-} \\
& =\sum_{Z} p\left(\mid X, \theta^{t} \log p \ll, Z ; \theta_{-}\right.
\end{aligned}
$$

This can be interpreted as filling in all possible values for the missing data $Z$ to give rise to the complete data, then we compute its log-likelihood having the component parameters $\theta$ set fixed, since not all possible values for the missing features are equally good, the goodness of a particular way of filling in $Z=z$ is determined by how likely the random variable $Z$ take the value $z$ given the observed data $X$ and the current parameter $\theta^{t}$

An improved parameter estimate at iteration $t+l$ is obtained by maximizing the Qfunction with respect to $\theta$.

$$
\theta^{t+1}=\arg \max _{\theta} Q\left(\theta ; \theta^{t}\right)
$$

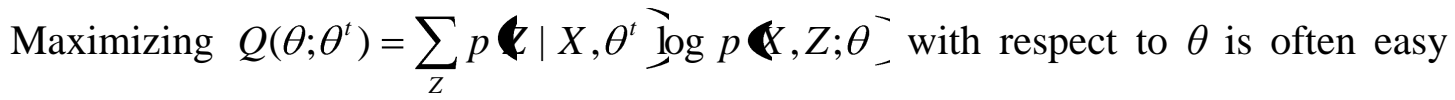
because maximizing the complete data $\log$-likelihood $\log p \ll, Z ; \theta_{-}^{-}$is assumed to be easy as long as we have its closed form, in the Gaussian mixture case, it is the weighted sum of the Gaussian pdfs. The EM algorithm takes its name because it alternates between 
the E-step (expectation) and the M-step (maximization), where the Q-function is computed in the E-step then maximized with respect to $\theta$ in the M-step.

From the computation viewpoint, the E-step computes the posterior probability $p\left(X, \theta^{t}\right.$, based on the current parameter estimate and the observed data which answer the question of what is the probability that $Z=z$ is the true hidden data given the current

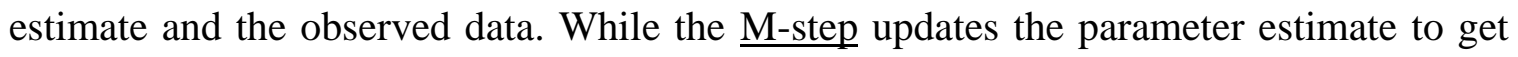
$\theta^{t+1}$ based on update equations derived analytically by maximizing $Q\left(\theta ; \theta^{t}\right)$. Hence the EM algorithm requires an initial guess $\theta^{0}$ for the parameter. Each iteration of the E-step and M-step is guaranteed to increase the log-likelihood of the observed data $\log p(X \mid \theta) \log \sum_{z} p \mathbb{X}, Z \mid \theta_{-}^{-}$until a local maximum is reached.

\subsection{Gaussian Mixture}

The missing data are the component labels $\left\{z_{1}, z_{2}, \ldots, z_{n}\right\}$ and the parameter vector is $\theta=$ $\left\{\alpha_{1}, \alpha_{2}, \ldots, \alpha_{k}, \mu_{1}, \mu_{2}, \ldots, \mu_{k}, \Sigma_{1}, \Sigma_{2}, \ldots, \Sigma_{k}\right\}$

\subsection{The E-Step}

The E-step computes the posterior probability of the missing data which answers the question of what is the probability that given $x_{i}$ the component that generated it is $j$ ? This is answered for each data point $x_{i}$ and for each Gaussian component $j$ as follows; 


$$
\begin{aligned}
r_{i j}=p \quad z_{i}=j \mid x_{i}, \theta & =\frac{p x_{i}, z_{i}=j \mid \theta}{p\left(x_{i} \mid \theta\right)} \\
& =\frac{p x_{i}, z_{i}=j \mid \theta}{p\left(x_{i}, z_{i}=1 \mid \theta\right)+\ldots+p\left(x_{i}, z_{i}=j^{\prime} \mid \theta\right)+\ldots+p\left(x_{i}, z_{i}=k \mid \theta\right)} \\
& =\frac{p x_{i}, z_{i}=j \mid \theta}{\sum_{j^{\prime}=1}^{k} p\left(x_{i}, z_{i}=j^{\prime} \mid \theta\right)} \\
& =\frac{\alpha_{j} p x_{i}, \mu_{j}, \sum_{j}}{\sum_{j^{\prime}=1}^{k} \alpha_{j^{\prime}} p x_{i}, \mu_{j^{\prime}}, \sum_{j^{\prime}}}
\end{aligned}
$$

\subsection{The M-Step}

The M-step maximized $Q\left(\theta ; \theta^{t}\right)=\sum_{Z} p \ll \mid X, \theta^{t} \log p \varangle, Z ; \theta_{-}^{-}$with respect to different parameters in the parameter vector $\theta$ in order to obtain the update equations.

Recall that $p x, \mu_{j}, \sum_{j}=\frac{1}{2 \pi^{\frac{d}{2}}\left|\sum_{j}\right|^{\frac{1}{2}}} \exp \left(-\frac{1}{2} x-\mu_{j}{ }^{T} \sum_{j}^{-1} x-\mu_{j}\right)$, the Q-function can be rewritten as follows: 


$$
\begin{aligned}
& Q\left(\theta ; \theta^{t}\right)=\sum_{Z} p Z \mid X, \theta^{t} \log p \quad X, Z ; \theta \\
& =\sum_{i=1}^{n} \sum_{j=1}^{k} p \quad z_{i}=j\left|x_{i}, \theta^{t} \log p \quad x_{i}, z_{i}=j\right| \theta \\
& =\sum_{i=1}^{n} \sum_{j=1}^{k} p \quad z_{i}=j \mid x_{i}, \theta^{t} \log \left[\begin{array}{llll}
p & z_{i}=j \mid \theta & p & x_{i}
\end{array} \mid z_{i}=j, \theta\right] \\
& =\sum_{i=1}^{n} \sum_{j=1}^{k} r_{i j} \log p \quad z_{i}=j\left|\theta+\log p \quad x_{i}\right| z_{i}=j, \theta \\
& =\sum_{i=1}^{n} \sum_{j=1}^{k} r_{i j} \log \alpha_{j}+\log p \quad x_{i}, \mu_{j}, \sum_{j} \\
& =\sum_{i=1}^{n} \sum_{j=1}^{k} r_{i j}\left(\log \alpha_{j}+\log \left(\frac{1}{2 \pi^{\frac{d}{2}}\left|\sum_{j}\right|^{\frac{1}{2}}} \exp \left(-\frac{1}{2}\left(x_{i}-\mu_{j}\right)^{\top} \sum_{j}^{-1}\left(x_{i}-\mu_{j}\right)\right)\right)\right. \\
& =\sum_{i=1}^{n} \sum_{j=1}^{k} r_{i j} \log \alpha_{j}+\sum_{i=1}^{n} \sum_{j=1}^{k} r_{i j}\left(-\frac{d}{2} \log (2 \pi)-\frac{1}{2} \log \left|\sum_{j}\right|-\frac{1}{2}\left(x_{i}-\mu_{j}\right) \sum_{j}^{-1}\left(x_{i}-\mu_{j}\right)\right. \\
& =\sum_{i=1}^{n} \sum_{j=1}^{k} r_{i j} \log \alpha_{j}-\frac{d}{2} \log (2 \pi) \sum_{i=1}^{n} \sum_{j=1}^{k} r_{i j}-\frac{1}{2} \sum_{j=1}^{k} \log \left|\sum_{j}\right| \sum_{i=1}^{n} r_{i j} \\
& -\frac{1}{2} \sum_{i=1}^{n} \sum_{j=1}^{k} r_{i j}\left(x_{i}-\mu_{j}\right) \sum_{j}^{-1}\left(x_{i}-\mu_{j}\right)
\end{aligned}
$$

To maximize $Q\left(\theta ; \theta^{t}\right)$ with respect to $\mu_{l}$, set the gradient to zero as follows;

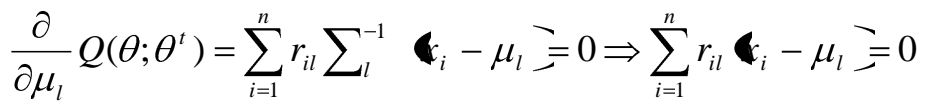

$$
\begin{aligned}
& =\sum_{i=1}^{n} \mathbf{Q}_{i l} x_{i}-r_{i l} \mu_{l} \mathcal{-}=\sum_{i=1}^{n} r_{i l} x_{i}-\sum_{i=1}^{n} r_{i l} \mu_{l} \\
& =\sum_{i=1}^{n} r_{i l} x_{i}-\mu_{l} \sum_{i=1}^{n} r_{i l} \\
& \Rightarrow \hat{\mu_{l}}=\frac{\sum_{i=1}^{n} r_{i l} x_{i}}{\sum_{i=1}^{n} r_{i l}}
\end{aligned}
$$

This is the update equation for the new $\mu_{l}$ at $(t+1)$-th iteration where $r_{i l}$ is computed based only on the parameter at the $t$-th iteration. 
Similarly, we have;

$$
\begin{gathered}
\hat{\sum_{l}=} \frac{\sum_{i=1}^{n} r_{i l} \boldsymbol{c}_{i}-\mu_{l} \boldsymbol{\triangleleft}_{i}-\mu_{l} \boldsymbol{\Psi}^{-}}{\sum_{i=1}^{n} r_{i l}} \\
\hat{\alpha_{l}}=\frac{\sum_{i=1}^{n} r_{i l}}{\sum_{j=1}^{k} \sum_{i=1}^{n} r_{i j}}
\end{gathered}
$$

Since EM is an iterative algorithm, it may end up in a local maximum instead of the global maxima of the log-likelihood of the observed data; hence a good initialization is needed. The EM algorithm may not be the most efficient algorithm for maximizing the $\log$-likelihood, however it is fairly easy to implement. The number of components $k$ in a Gaussian mixture is either specified by the user, or advanced techniques can be used to estimate it based on the available data. In summary, a mixture of Gaussian can be viewed as a middle-ground in terms of flexibility and memory requirements for parameter storage between the non-parametric approaches and a single Gaussian fitting.

While many of the computer vision applications use the histogram information, this paper is not restricted to any particular type of information; hence, the E-step and M-step of the EM algorithm is applied using the kernel definition in Eq (2.23) with the restrictions. Given an ensemble of data, a histogram may be obtained as an initial step in the estimation process. 


\section{EM Algorithm for Gaussian Mixture}

1. Start with an initial guess of the parameter vector $\theta^{0}=\left\{\alpha_{1}, \alpha_{2}, \ldots, \alpha_{k}, \mu_{1}, \mu_{2}, \ldots, \mu_{k}, \Sigma_{1}, \Sigma_{2}\right.$, $\left.\ldots, \Sigma_{k}\right\}$.

2. Set $\mathrm{t}=0$

3. While the log-likelihood of the observed data $\sum_{i=1}^{n} \log p \mathbf{C}_{i} \mid \theta^{t^{-}}$is still increasing, do:

a. Perform the E-step by computing $r_{i j}=p \quad z_{i}=j \mid x_{i}, \theta=\frac{\alpha_{j} p x_{i}, \mu_{j}, \sum_{j}}{\sum_{j^{\prime}=1}^{k} \alpha_{j^{\prime}} p x_{i}, \mu_{j^{\prime}}, \sum_{j^{\prime}}}$.

b. Perform the M-step by re-estimating the parameter using the following update equations:

$$
\begin{aligned}
& \hat{\alpha_{l}}=\frac{\sum_{i=1}^{n} r_{i l}}{\sum_{j=1}^{k} \sum_{i=1}^{n} r_{i j}} \\
& \hat{\mu}_{l}=\frac{\sum_{i=1}^{n} r_{i l} x_{i}}{\sum_{i=1}^{n} r_{i l}} \\
& \hat{\sum}_{l}=\frac{\sum_{i=1}^{n} r_{i l} \boldsymbol{\bigotimes}_{i}-\mu_{l} \boldsymbol{\triangleleft}_{i}-\mu_{l}{ }^{\mathbf{\top}} \text { - }}{\sum_{i=1}^{n} r_{i l}}
\end{aligned}
$$

c. Form $\theta^{t+1}$ based on the re-estimated parameters for each Gaussian component.

d. $\quad$ Set $\mathrm{t}=\mathrm{t}+1$

Figure 21_shows the fitting of Gaussian kernels to one of the probability density functions (pdf) of the bimodal histograms in Figure 11 obtained from a typical CT slice. The distinct separation of modes is the major reason for expected high performance of the EM algorithm. The fitting may be enhanced using variable number of Gaussians and we 
may employ the approach of [35] for automatic estimation of the number of terms in the linear model. Again, the distinct bi-modal pattern of the histograms (see Fig 11) makes it easy for employing a typical EM algorithm.

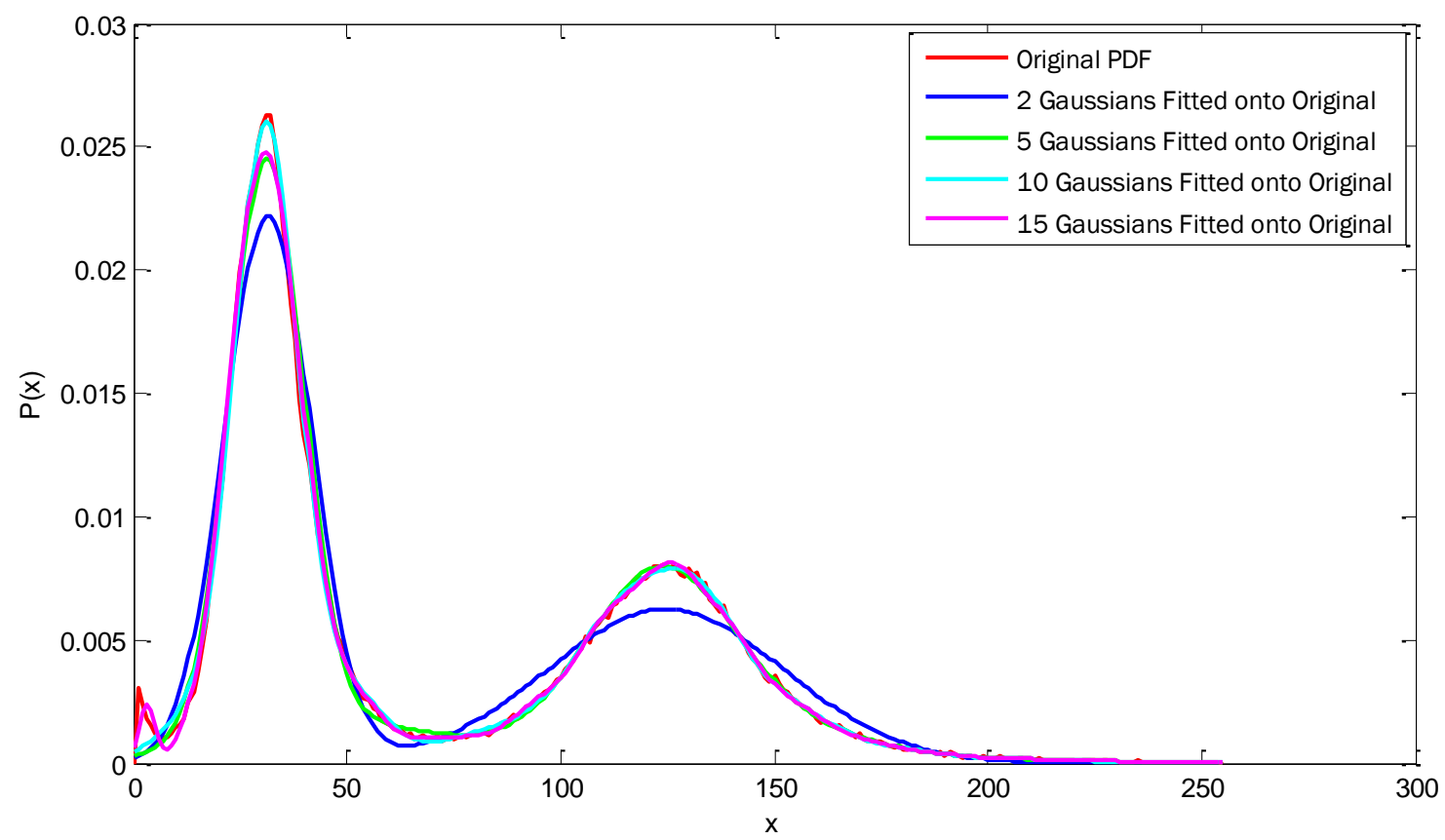

Figure 21: Fitting a linear model to the pdf of a typical lung CT scan

\section{E. SUMMARY}

This chapter considered the fundamental steps of image modeling that is used in this thesis. In particular, we studied the preprocessing component, which is conducted prior to the segmentation of the lung tissue and the nodule detection to follow. We also examined the intensity models of the gray scale in typical lung segmentation. The E-M algorithm is used as a major step in the segmentation approach to isolate the lung tissues from the 
thoracic region. The contribution of this chapter is mainly noise analysis of the ELCAP data, performance evaluation of the Weiner and anisotropic diffusion filters, and modeling the bi-modal intensity histogram using a simplified linear model. 


\title{
CHAPTER 3
}

\section{IMAGE SEGMENTATION}

\begin{abstract}
$\underline{\text { A. Introduction }}$
Image segmentation, as the name implies, connotes dividing or separating the information content in an image (or volume of images) into recognizable classes. It is a very fundamental and important step in image analysis. The image formation process can be exploited in separating the classes. Intensity-based representations, as the output of all biomedical imaging modalities, leave the gray scale or pixel/voxel values as the main source of information. Image formation may be considered as indirect information about an image or a priori information. This a priori information may take the form of shape and texture. The image segmentation process takes advantage of all pieces of information in order to provide connected separable regions. One can expect that various segmentation approaches exist as the imaging modalities vary and the inherent characteristics of objects vary a great deal. These issues and other factors related to the accuracy of the imaging process and the intended application made image segmentation an important area of research since the dawn of digital picture processing in the late 1960s.

It would be a futile effort to survey the approaches developed in the past 50 years, which runs into tens of thousands of papers in the technical literature. Indeed, the Computer Vision and Image Processing Laboratory (CVIP Lab) has developed well-cited approaches for image segmentation for various imaging modalities and from various
\end{abstract}


mathematical points of views. We make use of these proven approaches in this thesis, given the fact that the thesis is a continuation of efforts on the Lab's research on computerized algorithms for automatic detection of lung nodules and their classifications into pathologies. We limit our scope to algorithms that are adequate for chest LDCT scans. For this purpose, the CVIP Lab has developed a number of highly sophisticated approaches including: 1) level set approach (e.g., Farag and Abdelmunim, 2004 [15]), 2) statistical approach (e.g., Farag et al. 2006 [36], Ali and Farag, 2008 [16]). The author of this thesis has been involved in developing a simplified statistical approach (e.g., Elhabian, Farag et al. 2008 [14]) that exploits the major chrematistics of the lung CT scans as we studied in Chapter 2. This chapter will focus on this approach and will briefly discuss the sophisticated algorithms of Farag and Abdelmunim [15], 2004 and Ali and Farag, 2008 [16].

In the way of a short and concise reference to some of the work in the literature that dealt specifically with lung segmentation from chest CT scans we refer to the following studies.

Hu et al. [37], used a thresholding technique based on the characteristics of the CT data. Brown et al. [38] integrated region growing and morphological operations with anatomical knowledge to segment lung volume. Sluimer et al. [39] used a segmentationby-registration scheme for automated segmentation of the pathological lung in CT. In that scheme, a scan with normal lungs is registered to a scan containing pathology. When the resulting transformation is applied to a mask of the normal lungs, segmentation is found for the pathological lungs. Although shape-based, or Atlas-based (e.g.,[40]), 
segmentation overcomes the problem of gray level inhomogeneities, the creation of a general 3D shape model of the lung is not an easy task in the 3D case. Conventional methods that perform lung segmentation in CT depend on a large contrast in Hounsfield units between the lung and surrounding tissues. Although these methods accurately segment normal lung tissues from LDCT, they tend to fail in case of gray level inhomogeneity, which results from the abnormal lung tissues.

Optimization based methods, such as graph cuts, have been used for segmentation of the lung tissue (e.g., [41][42]). Boykov and Jolly in [41] introduced an interactive segmentation framework for segmenting the lung tissue. In that work, the user must identify some voxels as object and others as background seeds. Then graph cut approach is used to find the optimal cut that completely separates the object seeds from the background seeds. Lombaert et al. [43] performed graph cuts on a low-resolution image/volume and propagated the solution to the next higher resolution level by only computing the graph cuts at that level in a narrow band surrounding the projected foreground/background interface. Although the results of these approaches looked promising, manual interaction was still required. Interactive segmentation imposes some topological constraints reflecting certain high-level contextual information about the object. Chen et al. [44] used morphological operations and graph cuts to segment the lung from radiographic images automatically. In that work, an outer boundary is initialized for each lung region by shrinking 10 pixels from the boundaries of both vertical halves of an image. This method does not work in axial CT slices, where there is a lung part in the middle of the image. Inner boundaries were obtained by dilating the "regional minimum". 
However, due to the inhomogeneity in the given image, there were many "regional minimums" so they selected a "regional minimum" based on a threshold. Chen et al. [44] used graph cuts to find the boundaries of each lung region between its inner and outer boundaries. The data penalty and discontinuity penalty were chosen to be inversely proportional to the gray levels difference of the neighborhood pixels. This selection will not work in axial CT lung slices due to their gray level inhomogeneities.

Once again, the above studies are just a sample of a very vast literature on the subject of image segmentation; see Sluimer et al. [13] for more exhaustive survey.

\section{B. Model-Based Lung Segmentation}

In this section, we describe a simplified image segmentation approach that exploits the intensity characteristics of lung CT scans. We also briefly refer to two well-established and sophisticated algorithms developed at the CVIP Lab.

Algorithm 1: A simplified model-based segmentation approach

In this algorithm ([14]), lung region segmentation is based on multi-level thresholding of a given CT slice. Figure 22 shows the average histogram of ELCAP CT slices where two main peaks arise due to lung parenchyma and fat/muscle regions. A threshold is chosen to maximize the separation of these two regions. The threshold is obtained through fitting a mixture of Gaussians over the density histogram using the Expectation Maximization algorithm (Chapter 2). Multi-level thresholding is used to first isolate the thoracic region from the CT slice background; the lung parenchyma is then extracted from the segmented thoracic region. 
Morphological dilation using a circular structuring element is then applied as a smoothing filter on the contour of the segmented lung region in order to avoid losing nodules which are attached to the lung walls.

To decrease the sensitivity of the segmentation result to the structuring element diameter, we apply it to the inner and outer lung region contour. After segmentation was completed small nodules attached to the pleural surface were found to no longer exist since these nodules were segmented as not belonging to the lung parenchyma. This operation resulted in $6.5 \%$ of the ground truth nodules to be excluded from further experimentations.

Figure 22 shows the average histogram of the CT slices for the ELCAP database and our block diagram of the segmentation algorithm is shown in figure 23 .

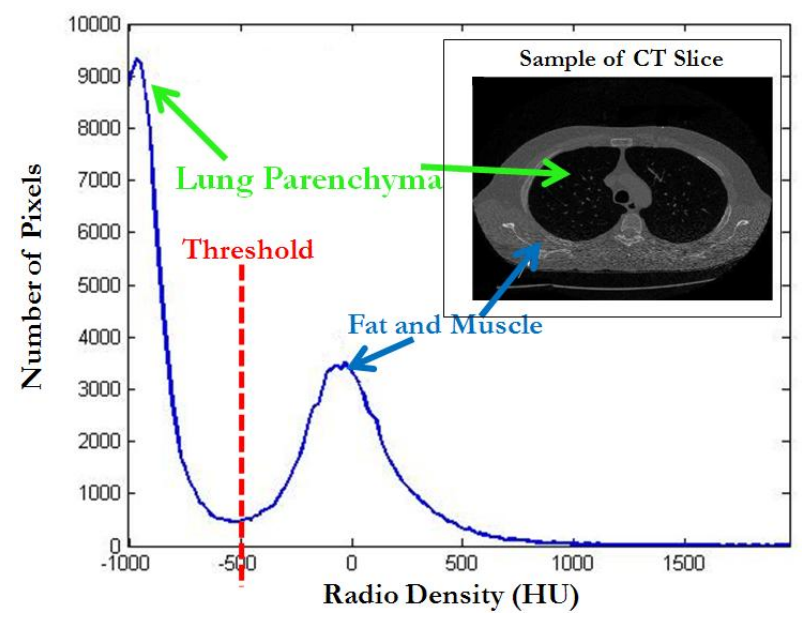

Figure 22: Average histogram of a CT slice from the ELCAP dataset. The average histogram of CT slices of the ELCAP database. Lung region (parenchyma) and $\mathrm{fat} / \mathrm{muscle}$ region constitute two dominant peaks in the histogram, in order to separate the lung region a threshold is chosen to maximize the separation between these two peaks. 


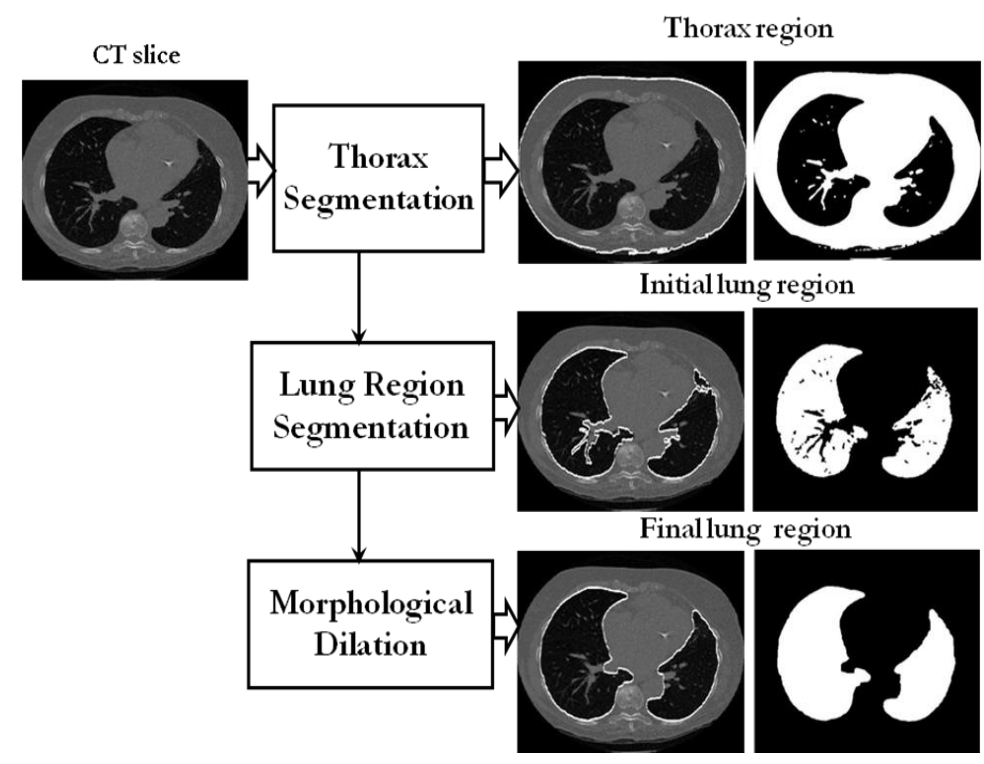

Figure 23: Block diagram of the segmentation algorithm.

Algorithm 1 is summarized as follows (modified version):

\section{Algorithm 1: Statistical Region Growing Approach; Elhabian, Farag et al [14]}

Step 1: Obtain the pdf histogram of the original image slice (the histogram will be bimodal as seen in figure 10). Fit numerous Gaussians over this pdf to obtain threshold to separate the thoracic region from the surrounding anatomy and background.

Step 2: Use connected component analysis and morphological operations to obtain the best mask image of the thoracic region.

Step 3: From the mask we multiply by original image to obtain an image of the chest wall and the lung region inside it.

Step 4: Repeat steps 2 and 3 this time to extract a mask for the inner lung region without the surrounding encasement.

Step 5: Morphological dilation is applied to image received in step 5 to smooth the edges to avoid losing nodules that can be on the pleural surface.

Step 6: Take the smoothed mask from step 6 to multiple with the original image and obtain the segmented lung region for the current image slice. 


\section{Algorithm 2: Graph Cuts-Based Approach [16]}

Ali and Farag [16] developed a statistical based segmentation algorithm for lung segmentation using composite random field modeling and employed the graph cuts approach to carry out the optimization of the resulting objective function which provides maximum a posterior (MAP) estimation of the desired segmentation.

The approach creates a weighted undirected graph with vertices corresponding to the set of volume voxels, and a set of edges connecting these vertices. The goal is to find the optimal segmented image, best labeling $\mathbf{f}$, by minimizing the following energy function:

$$
E(\mathbf{f})=\sum_{p \in \mathcal{P}} D_{p}\left(f_{p}\right)+\sum_{\{p, q\} \in \mathcal{N}} V\left(f_{p}, f_{q}\right)
$$

where $D_{p}\left(f_{p}\right)=-\ln \left(\mathrm{P}\left(I_{p} \mid f_{p}\right)\right)$, measures how much assigning a label $f_{p}$ (0 "background", or 1 "lung") to voxel $p$ disagrees with the voxel intensity $I_{p}$. The marginal density $\mathrm{P}\left(I_{p} \mid f_{p}\right)$ of each class $f_{p}$, is estimated by various approaches; for example, the linear model with Gaussian kernels (Chapter 2) or any of its modifications (e.g., [36]). The EM algorithm is used to estimate the model parameters. The second term of the above equation is the pairwise interaction model which represents the penalty for the discontinuity between voxels $p$ and $q$, which is defined as follows: $V\left(f_{p}, f_{q}\right)=\gamma$ if $f_{p} \neq f_{q}$, and 0 otherwise. A Gibbs-Markov random field (GMRF) with nearest 6-neighborhood was used to model the spatial interaction. For this specific model, the Gibbs potential $\gamma$ can be obtained analytically using the maximum likelihood estimator (MLE) for a GMRF (e.g., [36]).

\section{- Gray Level Probabilistic Model}


To initially label the lung volume and to compute the data penalty term $D_{p}\left(f_{p}\right)$, the EM algorithm is used to approximate the gray level marginal density of each class $f_{p}$, lung and background, using a linear model with Gaussian kernels; e.g.,

$$
\mathrm{P}\left(\mathrm{I}_{\mathrm{p}} \mid \mathrm{f}_{\mathrm{p}}\right)=\sum_{\mathrm{r}=1 \mathrm{~W}_{\mathrm{r}}}^{\mathrm{c}} \Phi_{r}\left(\mathrm{I}_{\mathrm{p}} \mid \Theta\right)
$$

where $\Phi(.,$.$) is a Gaussian density with parameter \theta \equiv(\mu, \sigma 2)$ with mean $\mu$ and variance $\sigma 2$. Again, various modifications to the linear model may be employed to enhance the quality of the estimate (e.g., [36][16]).

\section{- Spatial Interaction Model}

The homogenous isotropic pairwise interaction model which represents the penalty for the discontinuity between voxels $\mathrm{p}$ and $\mathrm{q}$ is defined as $V\left(f_{p}, f_{q}\right)=\gamma$ if $f_{p} \neq f_{q}$, and 0 otherwise. The simplest model of spatial interaction is the Markov Gibbs random field (MGRF) with the nearest 6-neighborhood. Therefore, for this specific model the Gibbs potential can be obtained analytically using the maximum likelihood estimator (MLE) for a generic MGRF[16]. The resulting approximate MLE of $\gamma$ is:

$$
\gamma^{*}=\left(K-\frac{K^{2}}{K-1} f_{\text {neq }}(f)\right)
$$

where

$$
f_{\mathrm{neq}}(\mathbf{f})=\frac{1}{\left|\mathbf{T}_{\mathbf{N}}\right|} \sum_{\{p, q\} \in \mathbf{T}_{\mathbf{N}}} \delta\left(f_{p} \neq f_{q}\right),
$$


$\mathrm{K}=2$ is the number of classes in the volume, the indicator function, $\delta(\mathrm{A})$ equals one when the condition $\mathrm{A}$ is true, and zero otherwise, $\mathrm{T}_{\mathrm{N}}$ is the family of the neighboring voxel pairs supporting the Gibbs potentials.

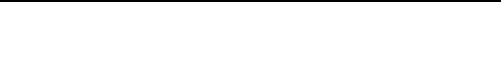

\section{Algorithm 2 (Ali-Farag [16])}

Given CT scan I, do:

1. Estimate the marginal densities of the lung and its background using model (2),

2. Produce an initial labeled volume $f$ using the intensity model's threshold,

3. Estimate an initial value of the interaction parameter $\gamma$ from $f$ using (3),

4. Integrate the intensity model (2), and GMRF model with interaction parameter (3) within the Bayesian framework of Maximum-A-Posteriori (MAP) estimation which equivalent to minimize the energy (1),
}

5. Minimize the energy (1) using Graph Cuts approach

6. Update the estimation of the interaction parameter $\gamma$

7. Repeat 5, 6, and 7 until the desired segmentation.

\section{Algorithm 3: Level Set Approach [15]}

Level set methods were first introduced by Osher and Sethian, 1988 [45]. Their goal is to handle topological merging and breaking, to work in any number of space dimensions. Also their algorithm is used in Hamilton-Jacobi type problems. Active contours were introduced by Kass, Witkins and Terzopoulos, 1987 [46] for segmenting objects in images. These algorithms were based on curve evolution and level set method. The basic idea is to represent contours as the zero 
level set of an implicit function defined in a higher dimension, referred to the level set function, and to evolve the level set function according to a partial differential equation (PDE). This method presents several advantages (e.g., Sethian, 1999 [47]) over the traditional parametric active contours. The contours represented by the level set function may break or merge naturally during the evolution, and changes are automatically handled. Another advantage is that the level set function always remains a function on a fixed grid, which allows efficient numerical schemes. The variational level set methods are more convenient than pure PDE driven level set methods because region-based information and shape-prior information are directly formulated in the level set domain. Hence, variational level set methods produce more robust results. For instance, Chan and Vese, 2001 [48] proposed an active contour model using a variational level set formulation.

Deformable models have had great success in medical imaging and computer vision. However, the disadvantage of this method is that the initial contour should be close to the final one. The method has some problems with topological changes of a complex structure (e.g., Abdelmunim and Farag, 2005 [49][50]). A signed distance map is used to handle complex rigid transformations with different scaling $\boldsymbol{\bigotimes}_{x}, s_{y}, s_{z}$, rotation $\boldsymbol{Q}_{x}, \theta_{y}, \theta_{z}$, and translation $\boldsymbol{\bigotimes}_{x}, t_{y}, t_{z}$, parameters of the shape registration.

The distance maps result in a more adequate energy function that obtains the transformation parameters. Also a shape-based PDE approach is included in this method. Hence, we do not need to tune the weighting coefficients. The first function is built as a function of the signed distance maps of the training shapes in a form of a parametric shape model. The second one is the segmentation of the region of interest based on the color value. The last function represents the evolving shape resulting from the combination of the color and the shape information. The energy function is obtained to measure the difference between the shape model and the color functions. 
The shape and the pose parameters are required to minimize this energy in a gradient descent approach (e.g., Farag and Abdelmunim 2004 [16]).

Farag and Abdelmunim [49][50] introduced a new level function defined as a vector distance rather than a scalar method. The level set function $\Phi$ is used to represent the evolving region. These representing shapes are invariant to translation and rotation. Given a curve/surface $V$ that represents boundaries of a certain shape, the following level set function can be defined as,

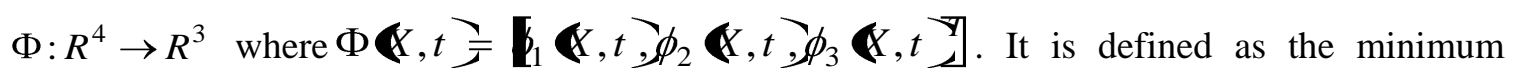
Euclidean distance between the point $X=\left\lfloor\left[, y, z^{T}\right]\right.$ and a curve/surface $V$. The evolving region is a propagating front embedded as the zero level or a higher dimensional function $\Phi$ [49]. The continuous change of the projections of $\Phi$ is described as

$$
\frac{d}{d t} \phi_{i}+\left|\nabla \phi_{i}\right| F_{i}=0 \quad i=1,2,3 .
$$

where $F$ is a vector velocity function depending on the local curvature of the front and on the external features related to the input image [49]. The parameter $\Phi$ deforms iteratively according to $F$. The position of the front is given at each iteration step by using the following equation:

$$
\Phi \ll, y, z, t=0
$$

F can be defined as

$$
F=\left[-\varepsilon k_{1}, v-\varepsilon k_{2}, v-\varepsilon k_{3}^{\top}\right]
$$

where $v=1$ or $v=-1$ for contracting or expanding the front, respectively, $\varepsilon$ is a smoothing coefficient smaller or equal to 1 , and $k_{i}$ is the local curvature defined for the corresponding projection function $\Phi_{i}$ where $i=1,2,3$. 
The intensity segmentation is described by the function $\Phi_{i}$ which changes based on (eq.3.4). If the point belongs to the associated object, the front region expands, otherwise it contracts. The point classification is based on the Bayesian decision at point at $X$. The parameter $v_{i}$ for each point is replaced by the function $v_{i} X_{-}^{-}$can be defined as follow:

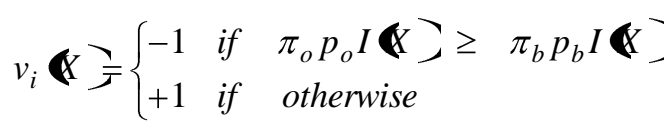

where $\pi$ is the region prior probability, $p \backslash$ is the probability density function (pdf) for the object $\mathbf{Q}_{-}$and the background $\boldsymbol{\varphi}_{-}$, and $I$ is the image data.

Each region is defined by a Gaussian distribution with adaptive parameters as follows [49]:

$$
\begin{aligned}
& \pi_{o}=\frac{\int_{2} H\left(\phi_{g} d \Omega\right.}{\int_{\Omega} d \Omega}, \pi_{b}=\frac{\int_{\Omega} H \boldsymbol{k}_{g} d \Omega}{\int_{\Omega} d \Omega} \\
& \mu_{o}=\frac{\int_{2} H \ll \phi I d \Omega}{\int_{\Omega} H \& \phi \lambda \Omega}, \mu_{b}=\frac{\int_{2} H \notin \mathrm{I} d \Omega}{\int_{\Omega} H \bigotimes \lambda \Omega}
\end{aligned}
$$

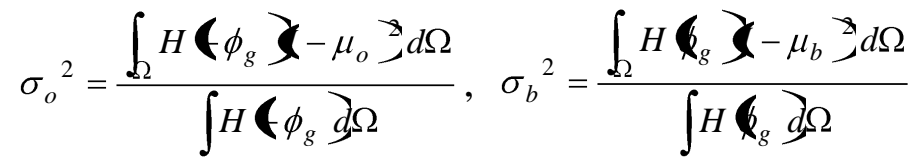

where $H$ is the Heaviside step function.

$\Phi_{1}, \Phi_{2}$, and $\Phi_{3}$ are the projections of the distance in the coordinates directions negative inside the curve/surface, positive outside and zero on the boundary ([15][49][50]). The algorithm can be summarized as follows: 


\section{Algorithm 3: Farag-Abdelmunim [15][50]}

Step 1: Manually select the initial seeds inside the region of interest.

Step 2: For the bi-model in this work, assume object and background classes have Gaussian distribution.

Step 3: Obtaining edges.

Step 4: Iteratively estimate the mean and standard deviation of the object and background (Eqs. 3.8- 3.10).

Step 5: For each pixel do the Bayesian decision (Eq. 3.7)

Step 6: Repeat steps 4 and 5 until the iteration is ended.

\section{Evaluation of Three Segmentation Algorithms}

The above three algorithms were evaluated on the ELCAP dataset in terms of accuracy and time of execution. The accuracy is determined with respect to a manually segmented sample data set, which will be called the ground truth. As our goal is to ultimately detect and classify nodules the ground truth segmentation was generated as follows:

1. An anisotropic diffusion filter (Chapter 2) was applied on an ensemble of size 6 slices of the ELCAP data with known nodules. We used a filter of width $8 \times 8$.

2. We manually segmented the 19 slices in step 1 using our best judgment in cropping out the lung tissues from the rest of the chest and thoracic regions. This data set is called the ground truth for segmentation.

3. We applied the three segmentation methods on the filtered data in step 1.

Figure 24 shows the results of the segmentation algorithms. 
The quality of segmentation, with respect to the ground truth, is measured using three measures: 1) the sum square difference (SSD), 2) the mutual information (MI), and 3) the number of visible nodules in the segmentation output. We have also applied the MI measure of the ground truth vs. the ground truth in order to calibrate that scale. The output of these comparisons is tabulated in Table 1 and Table 2.

\begin{tabular}{|c|c|c|c|c|}
\hline Original & Groundtruth & Algorithm 1 & Algorithm 2 & Algorithm 3 \\
\hline & & & \\
\hline & & & \\
\hline
\end{tabular}

Figure 24: Output of the segmentation algorithms with respect to ground truth on sample slices from the ELCAP dataset. From left to right: Original, Manual Segmentation; Algorithm 1, Algorithm 2 and Algorithm 3. For visualization purposes the images were placed on a white background. 
Table 1: The Square Sum Difference (SSD) measure with respect to the ground truth

\begin{tabular}{|l|l|l|}
\hline \multicolumn{3}{|c|}{ Square Mean Difference } \\
\hline $\begin{array}{c}\text { Groundtruth vs. } \\
\text { Algorithm 1 }\end{array}$ & $\begin{array}{c}\text { Groundtruth vs. } \\
\text { Algorithm 2 }\end{array}$ & $\begin{array}{c}\text { Groundtruth vs. } \\
\text { Algorithm 3 }\end{array}$ \\
\hline 0.0152 & 0.0042 & 0.0106 \\
\hline 0.0144 & 0.0038 & 0.0065 \\
\hline 0.0139 & 0.0044 & 0.0060 \\
\hline 0.0054 & 0.0042 & 0.0026 \\
\hline 0.0114 & 0.0124 & 0.0064 \\
\hline 0.0102 & 0.0094 & 0.0064 \\
\hline
\end{tabular}

Table 2: The Mutual Information (MI) measure with respect to the ground truth

\begin{tabular}{|l|l|l|l|l|l|l|}
\hline \multicolumn{7}{|c|}{ Mutual Information and percentage } \\
\hline $\begin{array}{l}\text { Groundtruth vs. } \\
\text { Groundtruth }\end{array}$ & \multicolumn{7}{|c|}{ Groundtruth vs. Algorithm 1 } & \multicolumn{2}{l|}{ Groundtruth vs. Algorithm 2 } & \multicolumn{2}{l|}{ Groundtruth vs. Algorithm 3 } \\
\hline$-9.9434 \mathrm{e}+005$ & $-9.9996 \mathrm{e}+005$ & $99.4375 \%$ & $\begin{array}{l}-9.9689 \mathrm{e} \\
+005\end{array}$ & $99.7441 \%$ & $-9.9971 \mathrm{e}+005$ & $99.4626 \%$ \\
\hline$-9.9236 \mathrm{e}+005$ & $-9.9783 \mathrm{e}+005$ & $99.4519 \%$ & $\begin{array}{l}-9.9470 \mathrm{e} \\
+005\end{array}$ & $99.7649 \%$ & $-9.9554 \mathrm{e}+005$ & $99.6810 \%$ \\
\hline$-9.9221 \mathrm{e}+005$ & $-9.9754 \mathrm{e}+005$ & $99.4653 \%$ & $\begin{array}{l}-9.9483 \mathrm{e} \\
+005\end{array}$ & $99.7370 \%$ & $-9.9525 \mathrm{e}+005$ & $99.6944 \%$ \\
\hline$-1.0282 \mathrm{e}+006$ & $-1.0299 \mathrm{e}+006$ & $99.8327 \%$ & $\begin{array}{l}-1.0305 \mathrm{e} \\
+006\end{array}$ & $99.7819 \%$ & $-1.0295 \mathrm{e}+006$ & $99.8722 \%$ \\
\hline$-1.0215 \mathrm{e}+006$ & $-1.0255 \mathrm{e}+006$ & $99.6130 \%$ & $\begin{array}{l}-1.0278 \mathrm{e} \\
+006\end{array}$ & $99.3871 \%$ & $-1.0247 \mathrm{e}+006$ & $99.6911 \%$ \\
\hline$-1.0320 \mathrm{e}+006$ & $-1.0361 \mathrm{e}+006$ & $99.6019 \%$ & $\begin{array}{l}-1.0368 \mathrm{e} \\
+006\end{array}$ & $99.5359 \%$ & $-1.0354 \mathrm{e}+006$ & $99.6720 \%$ \\
\hline
\end{tabular}

The three algorithms maintained the visible nodules and Algorithm 1 is the fastest among the three algorithms; it also required the minimum intervention. Algorithms 2 and 3 are very sophisticated and are able to handle data sets that have more distortions and occlusions than the CT data we have. In the subsequent analysis, we will use Algorithm 1. 


\section{Summary}

In this chapter we examined the segmentation process which isolates the lung tissue from the rest of the chest and thoracic regions in the CT scans. We studied three algorithms developed in the CVIP Lab and evaluated their performance on welldescribed data set that is manually segmented. Accuracy of the algorithms, with respect to the ground truth, is measured in terms of square error distance (SED) and the mutual information (MI), maintaining the nodules, and the execution time. This comparison provided confidence that a simplified statistical method (statistical region growing), Algorithm 1, is adequate for subsequent analysis of lung nodule detection. 


\section{CHAPTER 4}

\section{LUNG NODULE MODELING AND AUTOMATIC DETECTION}

\section{A. Introduction}

The goal of computer-based nodule analysis methods is to assist the radiologists in early detection of doubtful nodules. Assistance means to be able to mimic what a physician does in detecting and judging doubtful nodules. As stated in the introduction, in the United States, lung cancer accounts for over $30 \%$ of all cancer-related deaths, resulting in over 160,000 deaths per year [51]. That is more than the annual deaths from colon, breast, pancreatic, prostate, and ovarian cancers combined. Lung cancer survival is strongly dependent on the pathologic stage at the time of diagnosis [52][53]. The hope is that early detection of lung cancer can improve the survival rate of this disease, thus research studies to reach an optimal detection rate is important. Should it becomes a standard practice (like annual psychical exams) that a LDCT scan be recommended for every person after a certain age an automatic way to analyze the scans will lend great benefit. Indeed, one of the major goals of screening studies is to generate the data for designing (including testing and validating) of computer based nodule detection algorithms.

CT images are analyzed by radiologist during the screening process to locate pulmonary nodules on patient CT images. This process requires the acquisition of CT chest images in a single breath hold, with 1-3mm axial collimation [54]. These thin slices can assist with the detection of small nodules that can be a representation for early 
stages of lung cancer. CT is an incredibly sensitive imaging modality available for lung cancer screening $[17,55]$, yet it produces large quantities of thin axial slice images per patient (512x512x400 12-bit data per volume is the typical size for a thin slice CT screening database [56]) that the radiologist must analyze, leading to high false-negative rates due to missed small nodules. Small nodules can be over-looked due to several main reasons: nodule characteristics (density, size and location), scanning technique (radiation dosage and slice thickness) and human error. The enhancement of CT imaging with respect to resolution, dose and scanning approaches has motivated researchers to design fully automated computer-aided diagnosis (CAD) systems [57] for optimum nodule detection.

The generalized framework for a CAD system consists of four main steps (see, Figure 25):

1) Filtering and normalization of the LDCT scans;

2) Segmentation of the lung regions (parenchyma) from the surrounding tissue;

3) Detection of lung nodules; and

4) Nodule classification as benign or malignant.

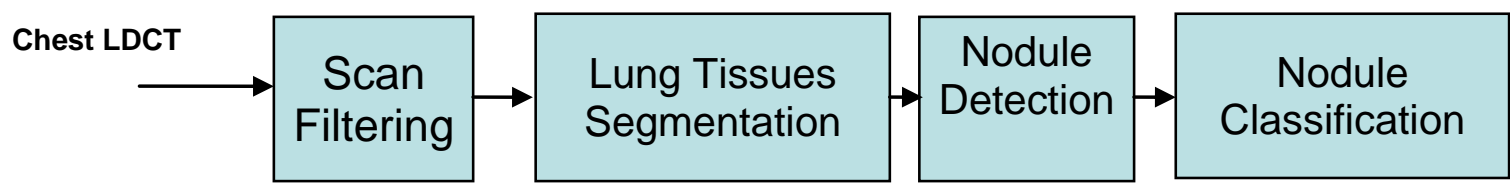

Figure 25: A block diagram of the major steps involved in computer-based analysis of LDCT of the chest in order to detect and classify doubtful lung nodules. 
In chapter 2 and 3 we examined the first two steps. This chapter deals with nodule detection and the following chapter will highlight some of the issues related to nodule classification. At this stage, we do not have a comprehensive database of nodules that allow rigorous analysis of features versus pathology; hence, the classification into certain pathology is not going to be addressed at great length in this thesis. We will, however, examine in great depth the nodule detection issue. We will use the ELCAP dataset [5] for nodule design and testing and will study the sensitivity of the template matching approach in terms of detection and reduction of false positives.

Since the early 90's various approaches for automated pulmonary nodule detection have been introduced. These approaches can be categorized as follows [54]: model based and density-based approaches. Template matching is one technique for model-based approaches which utilize a priori information of the size, intensity and shape of the nodules. Density-based approaches uses the fact that the lung parenchyma has relatively lower CT attenuation (density) than those of the lung nodules, thus they utilize image processing algorithms that rely on multiple thresholding [54], region growing and clustering [58].

In this chapter we use the ELCAP national database of lung CT scans where, each of the nodules are defined by radiologists and specified by a point on a $2 \mathrm{D}$ slice (the whole shape of the nodule(s) and 3D information for the ground-truth nodules is not provided). This chapter mainly focuses on template matching to detect candidate nodule. The effectiveness of template matching is measured by generating receiver operating characteristics (ROC) curves indicating the probability of detection (true positive rate) as 
a function of template parameters, by cross validation with respect to the ground truth, provided by radiologists. Results are reported for different template sizes, shapes and orientations (if applicable).

Section B of this chapter discusses segmenting the lung region from the surrounding tissue using a linear-based density estimation method, section $\mathrm{C}$ discusses template matching to identify the candidate nodules using numerous parametric template designs, section D presents our experimental results and section $\mathrm{E}$ is a summary of the chapter.

\section{B. Lung Regions Segmentation}

The process aims at isolating the lung tissues from the rest of chest and thoracic regions in the CT scans. This data reduction is intended to focus the attention on the lung tissues. Of course, some nodules may indeed appear in the thoracic region and may be of clinical importance; however, this thesis is focusing only on the lungs. Chapter 3

described three segmentation algorithms that have been well-developed and tested at the CVIP Lab. The methodologies developed in that chapter will be tested on the segmentation results from Algorithm 1 (Elhabian, Farag, et al., 2008 [14]).

\section{Nodule Modeling}

\section{Nodule Types}

A pulmonary nodule usually has a spherical shape; however, it can be perplexed by surrounding anatomical structures such as vessels and the pleural surface. We shall use 
the nodule classification of Kostis et al [10][12], which groups nodules into four categories:

i) well-circumscribed where the nodule is located centrally in the lung without being connected to vasculature;

ii) vascularized where the nodule has significant connection(s) to the neighboring vessels while located centrally in the lung;

iii) pleural tail where the nodule is near the pleural surface, connected by a thin structure;

iv) and juxta-pleural where a significant portion of the nodule is connected to the pleural surface.

See Fig. 26 for illustration.

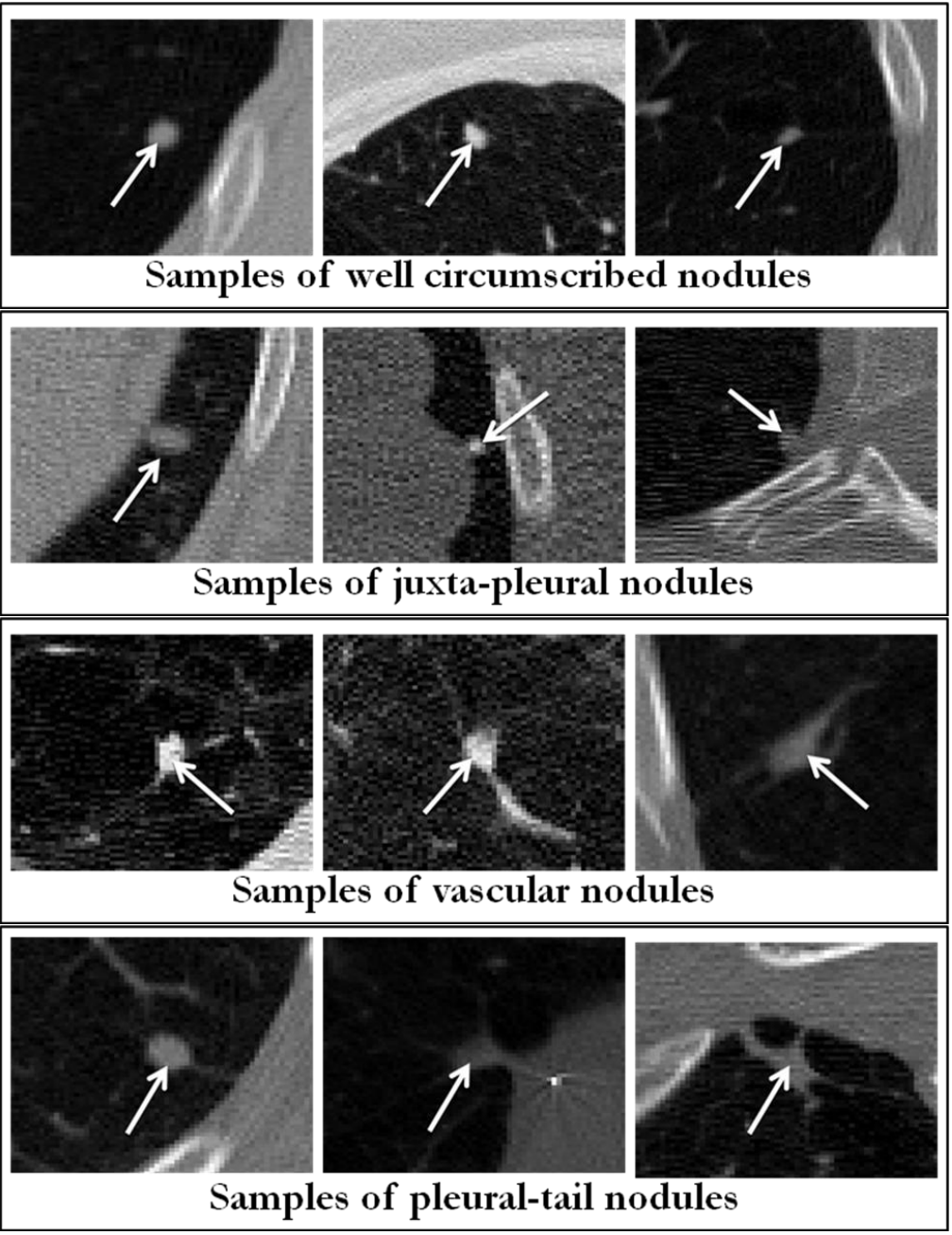

Figure 26: An ensemble of the four classes of pulmonary nodules segmented by experts. 


\section{Nodule Simulation}

In a CT scan the nodules can take various shapes and topologies, but the common characteristic amongst the nodules is the density distribution that tends to be concentrated around a region with an exponential decay (e.g., [58] [18][19][60]). To illustrate this behavior, Figure 27 shows the radial distance for each of the nodule types

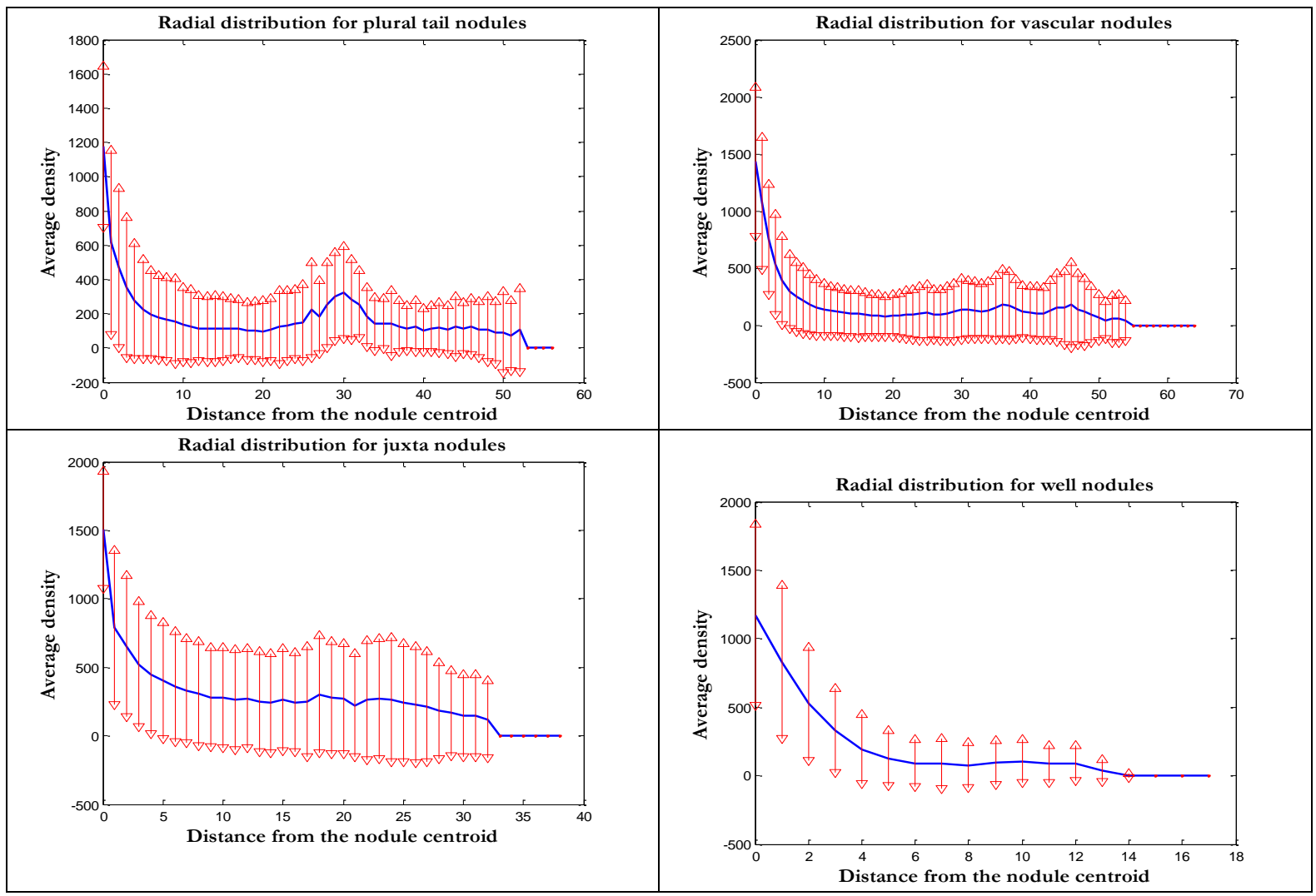

Figure 27: The distribution of the radial distance from the centroid of the nodules. Note the standard exponential behavior of the radial distance. This will be used in the design of templates as we will discuss in the following section.

Given this density distribution that tends to be concentrated around a region with an exponential decay, we may consider the nodule as a collection of pixels (voxels in 3D) 
with gray level distribution that is decaying exponentially from the centroid of its shape. In fact, we may use the average of the radial distances of the four types of nodules (Figure 28) in order to assign the gray level distribution for a given nodule shape.

Figure 28 shows the average distribution of $\mathrm{HU}$ densities for each nodule type. It can be observed that nodules' density distribution exhibit a bi-modal distribution (two dominant peaks) where the lower density mode represents regions surrounding the nodules and the higher density mode represents nodules densities

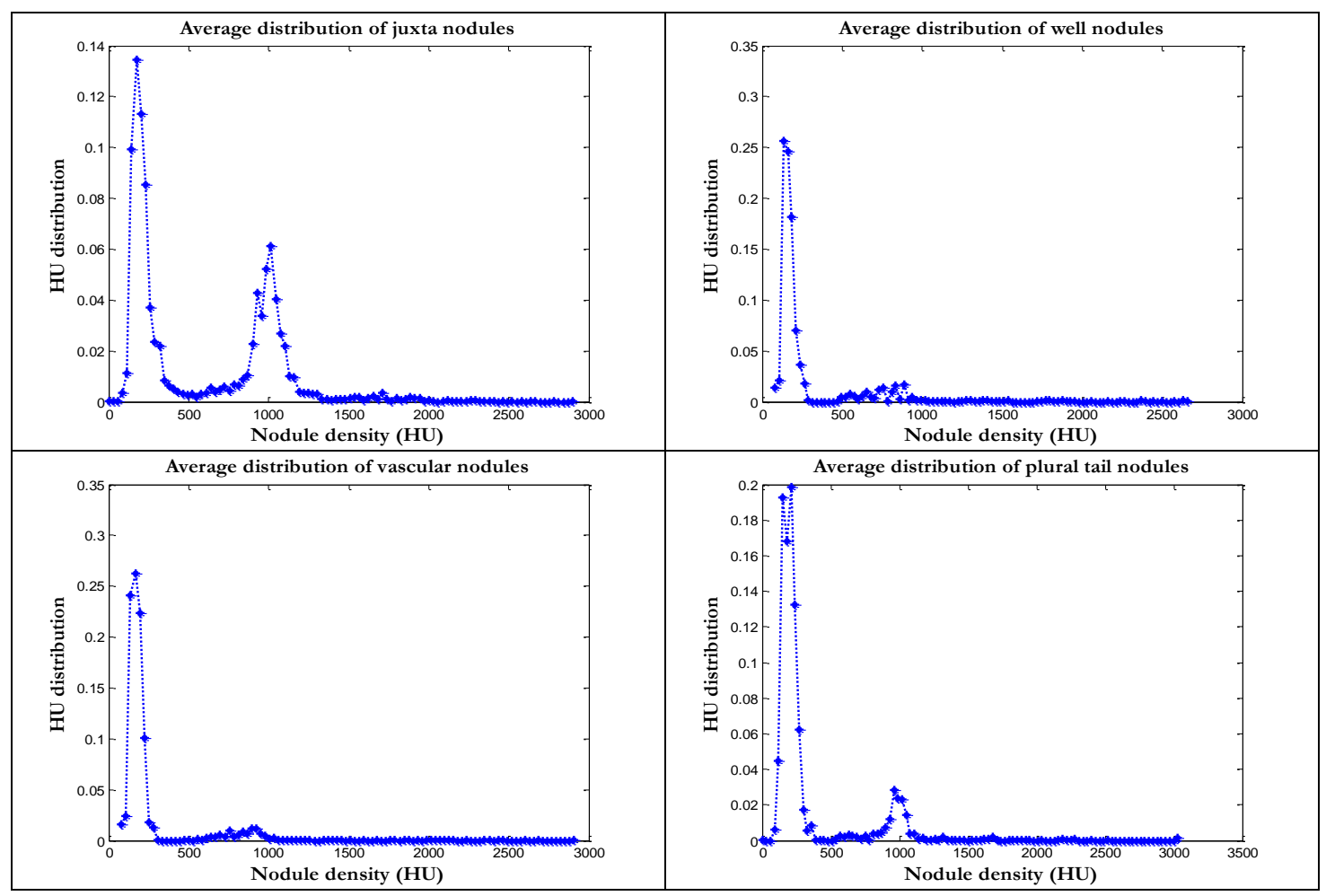

Figure 28: shows the average distribution of $H U$ densities for each nodule type.

Using EM algorithm, nodule $\mathrm{HU}$ density distribution can be modeled as a mixture of Gaussians with two dominant peaks. 
Figure 29 shows the marginal density distribution for the Juxta nodules and the regions surrounding the nodules, where two density values are taken into consideration for each nodule type for subsequent processing, they are the lowest and highest density values exhibited by the nodule region. We use the EM algorithm to separate the marginal densities of the nodule region from the surrounding region of the lung tissue.

So far we computed the density versus radial distance from nodule centroid, and the probability of having a certain density belonging to a nodule region. We can use this information to compute the HU density with respect to radial distances from nodule centroid. Figures 29 - 37 show the progression of empirical investigation of this thesis, for nodule modeling.

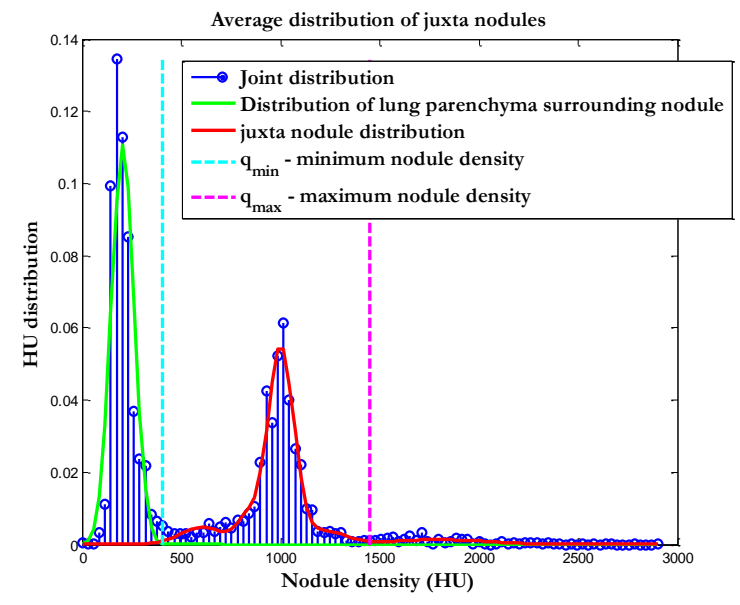

Figure 29: Histogram of the Juxta nodule type. 


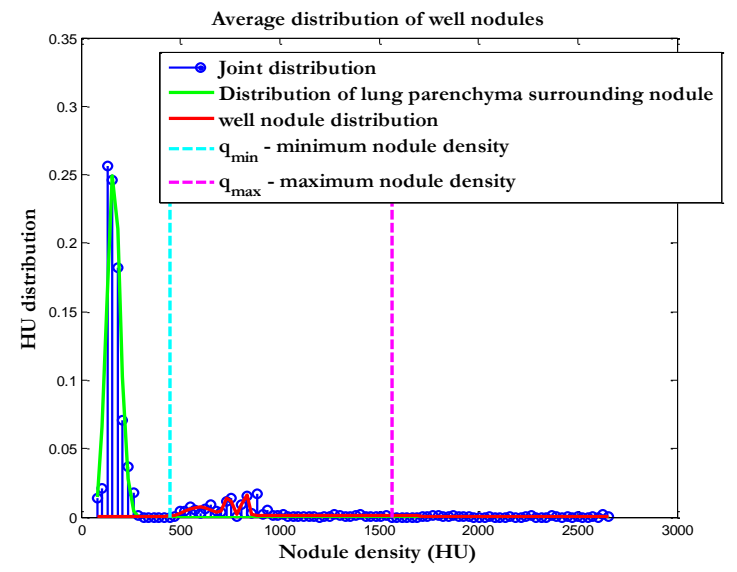

Figure 30: Histogram of the Well-Circumscribed nodule type.

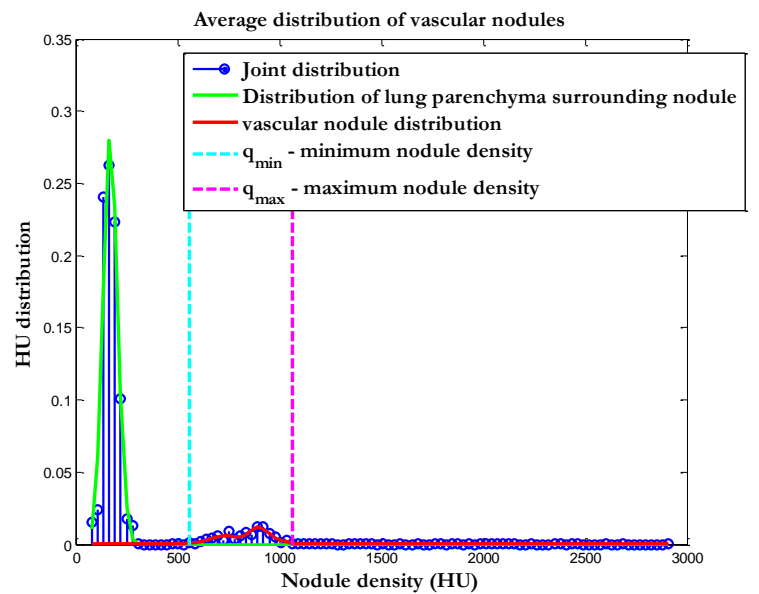

Figure 31: Histogram of the Vascular nodule type. 


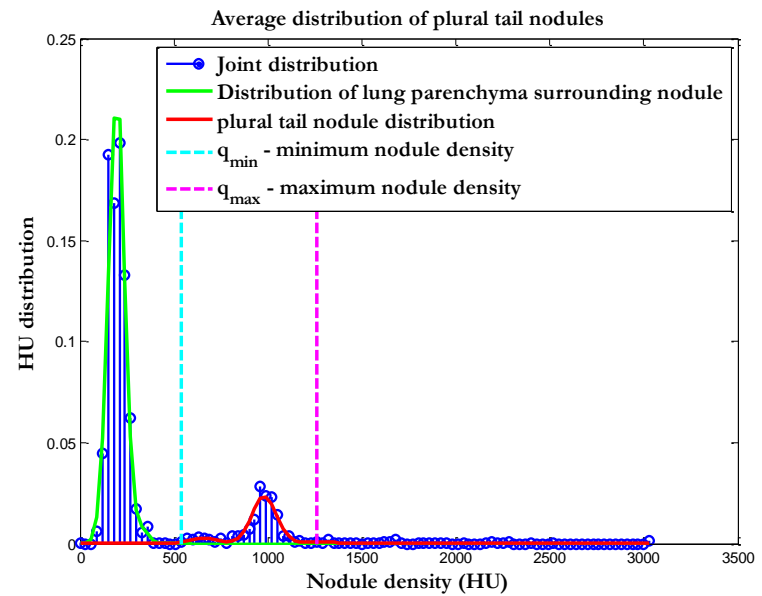

Figure 32: Histogram of the Pleural Tail nodule type.

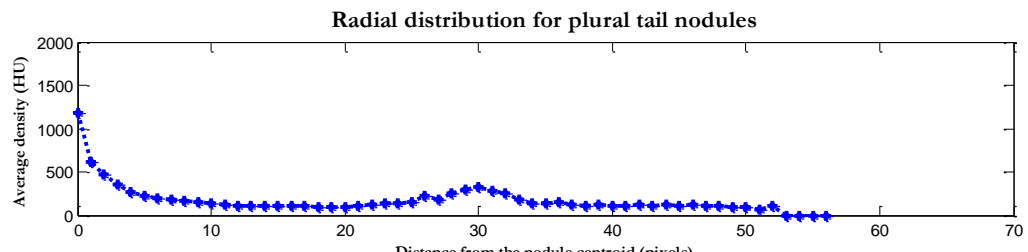

Average distribution of plural tail nodules
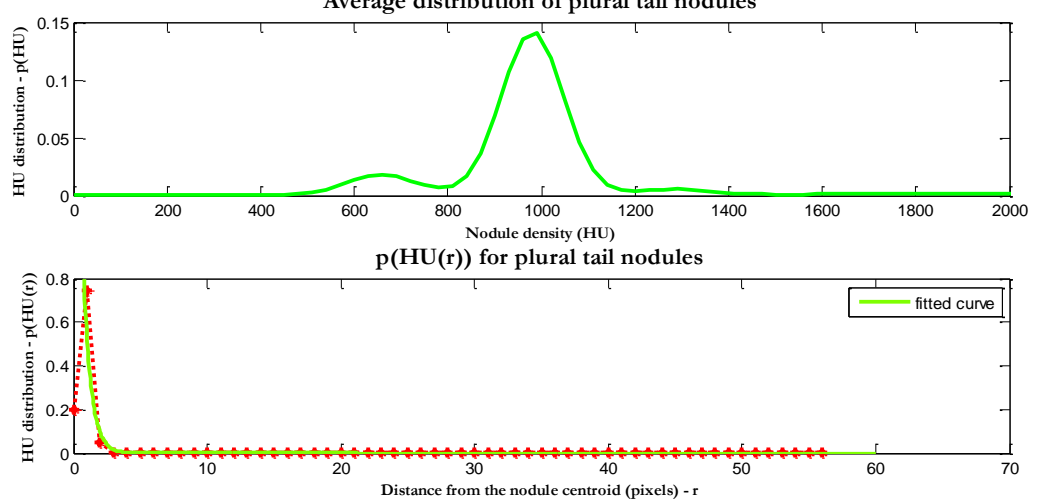

Figure 33: Probability density of the Radial distance of the Pleural Tail nodule. Note that the density is concentrated for distances less than 5 pixels from the centroid. 

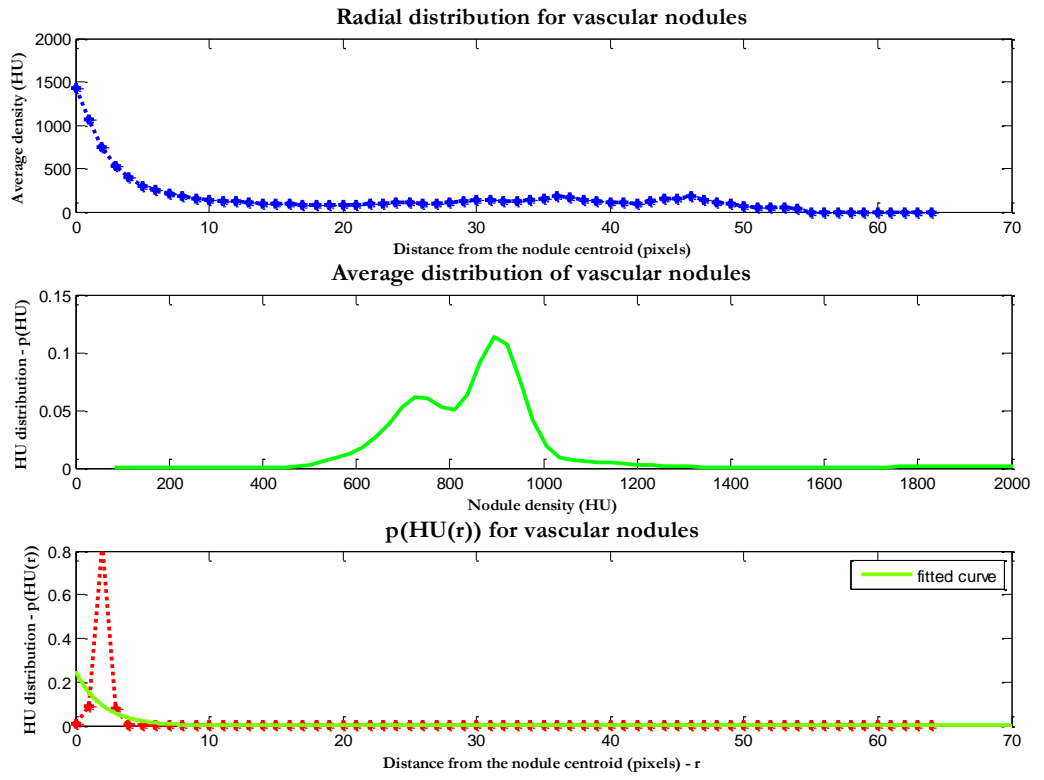

Figure 34: Probability density of the Radial distance of the Vascular nodule.
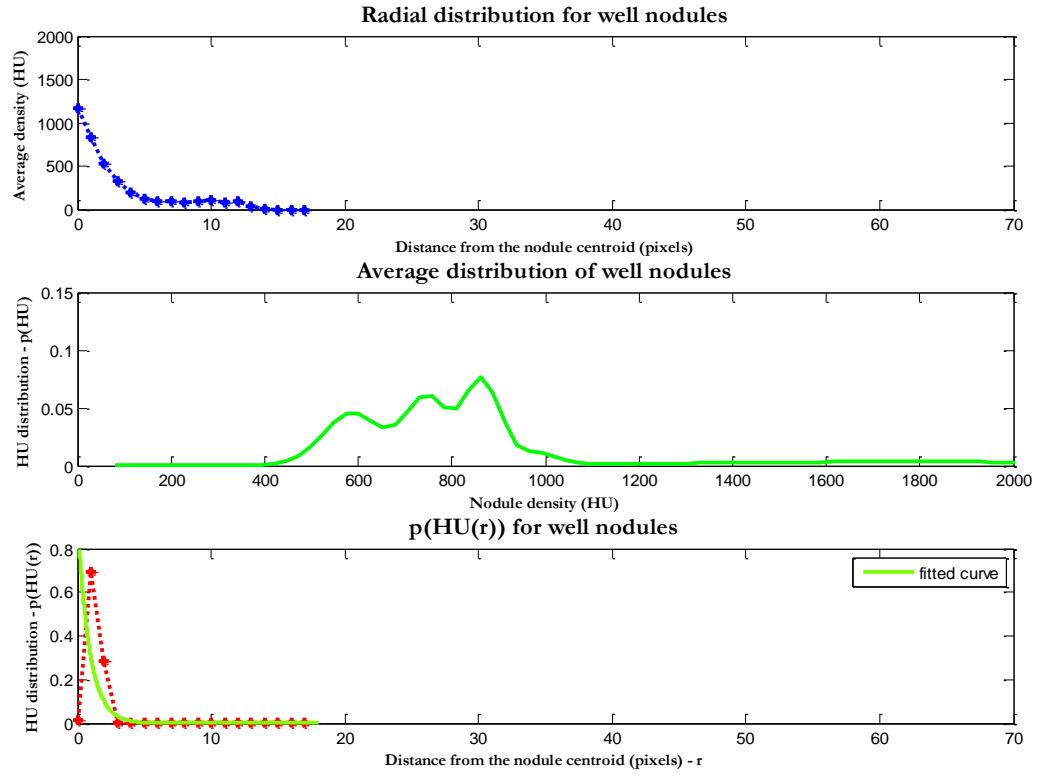

Figure 35: Probability density of the Radial distance of the Well-Circumscribed nodule. 

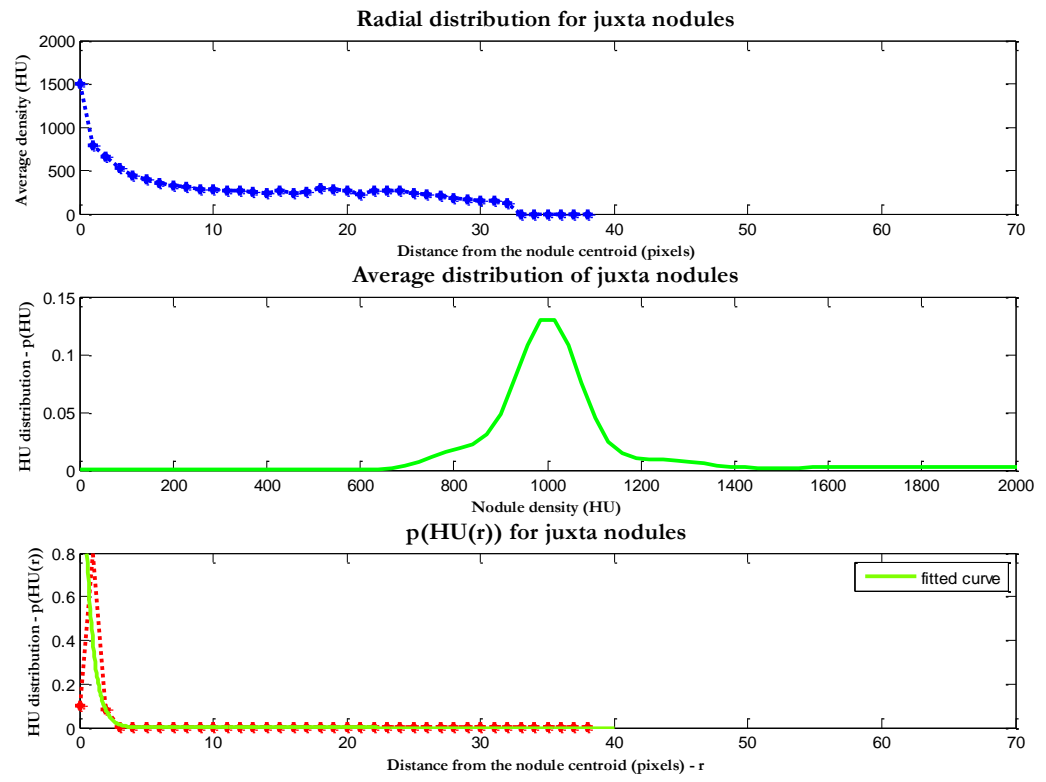

Figure 36: Probability density of the Radial distance of the Juxta nodule.

Figure 37 shows the average radial density distribution for all nodule types. Note that the density is concentrated in distances below 5 pixels from the centroid.

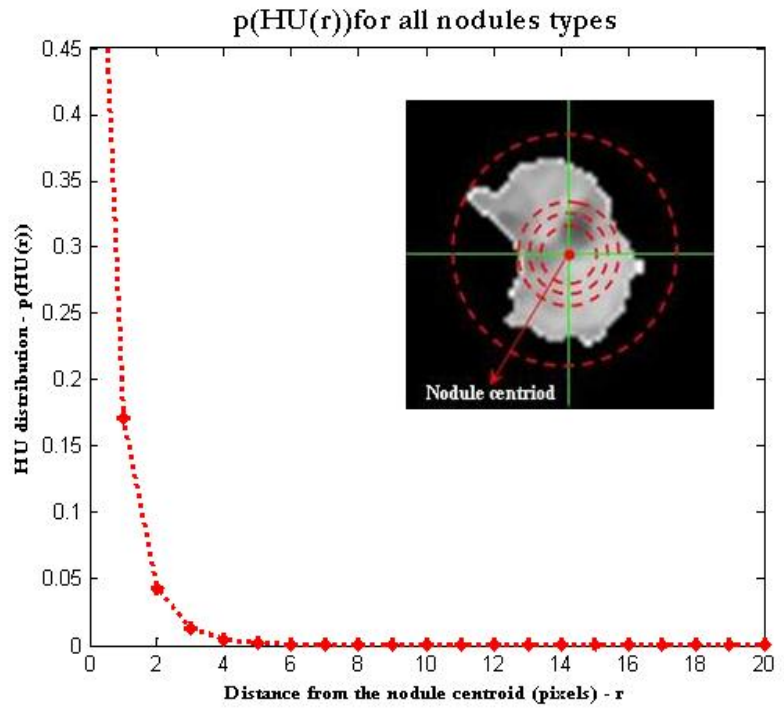

Figure 37: Average radial distance of four nodule types. 
The conclusions of this empirical investigation may be two folds:

1) It seems logical to model the nodule density distribution with respect to radial distribution from the nodule centroid as an exponential distribution.

2) As the density of the radial distance from the centroid is around 5 pixels, one can conclude that a template of radius 10 pixels $(5 \mathrm{~mm})$ will be adequate in terms of size to simulate a real nodule.

Given the range of nodule density distribution trained from the ground truth nodules (in the training stage) $\mathrm{q}_{\min }$ and $\mathrm{q}_{\max }$, from the centroid of the nodule (for any given shape), the intensity or HU, at a distance $r$ from the centroid, can be estimated by the following equations.

$$
\begin{aligned}
& q(r)=q_{\max } e^{-}, \quad 0 \leq r \leq R \\
& \rho=R\left(\operatorname{n}\left(q_{\max }\right)-\ln \left(q_{\min }\right)^{-1 / 2}\right.
\end{aligned}
$$

\section{Nodule Detection}

This section will discuss the types of parametric templates used for $2 \mathrm{D}$ and $3 \mathrm{D}$ template matching and the design process conducted to generate them. Then the template matching as a process for nodule detection is explained.

\section{Template Design}

The most common parametric nodule models in 2D are circular and semi-circular. In 3D the corresponding models would be spherical and hemi-sphere or cups. Figure 38 shows a few examples of such templates. In this thesis, for the 2D case the circular and semi- 
circular parametric templates (isotropic and non-isotropic) are used. The isotropic templates are defined in terms of the radius (size), $\mathrm{R}$, and the gray level distribution as a circular symmetric Gaussian function [58][14][18] while non-isotropic templates are defined by radius, gray level distribution and orientation.

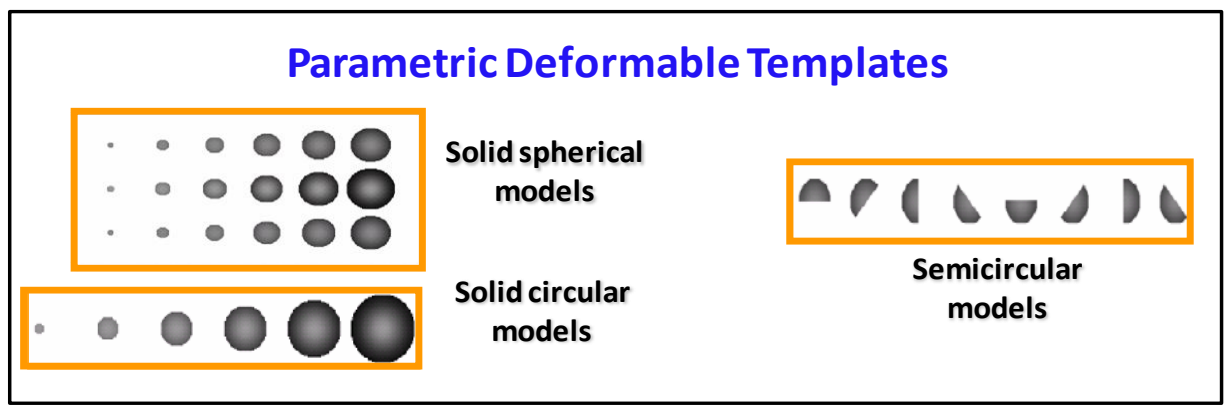

Figure 38: Samples of parametric templates of various sizes in $2 D$ and $3 D$.

Automatic generation of the gray level distribution of the nodules with known radius and histogram of nodule prototypes can be generated, for a given shape, using Equations 4.1 and 4.2 above. This is particularly simple to perform in the case of parametric templates (e.g., Figure 38), where given a diameter $R$ we only need to estimate the intensity of $\mathrm{HU}$ from these equations.

\section{Template Matching}

Template matching refers to the process of detecting an object with known prior information such as size, shape and orientation, where detection can be achieved by applying a filter, known as a template, having positive weights in a region that resembles the object to be detected and zeroes weights in other areas. If the result of the matching process between an unknown object and the template is sufficiently high, the unknown 
object is labeled as resembling the template, however due to image noise, spatial, amplitude quantization effects, and a priori uncertainty of the exact shape and structure of the object to be detected, an exact match rarely occurs. Hence normalized cross correlation is used which has a maximum value of unity that occurs if and only if the image function under the template exactly matches the template. i.e., the normalized cross-correlation of a template, $t(x, y)$ with a sub-image $f(x, y)$ is given as:

$$
N C C=\frac{1}{n-1} \sum_{x, y} \frac{(f(x, y)-\bar{f})(t(x, y)-\bar{t})}{\sigma_{f} \sigma_{t}}
$$

where $n$ is the number of pixels in template $t(x, y)$ and sub-image $f(x, y)$ which are normalized by subtracting their means and dividing by their standard deviations.

The probability density functions (pdf) of nodule and non-nodule pixels are computed using the normalized cross correlation coefficients resulted from templates with varying parameters (shape, size and orientation if applicable). Based on the Bayesian classification theory, the intersection between the pdf's of the two classes is chosen as the threshold separating the correlation coefficients resulted from nodule and non nodule pixels.

\section{E. Experimental Results}

\section{Dataset:}

This study is based on the ELCAP public database [5], which contains 50 sets of lowdose CT lung scans taken at a single breath-hold with slice thickness $1.25 \mathrm{~mm}$. The locations of the 397 nodules are provided by the radiologists, where $39.12 \%$ are juxta- 
pleural nodules, $13.95 \%$ are vascularized nodules, $31.29 \%$ are well-circumscribed nodules and $15.65 \%$ are pleural-tail nodules. The official reports indicate the mean nodule diameter to be $8.5 \mathrm{~mm}$ with standard deviation 3.6. The ELCAP database is of resolution $0.5 \times 0.5 \mathrm{~mm}[5]$.

\section{Basic Template Matching:}

To illustrate the effectiveness of the NCC measure for matching, the template matching was applied on neighborhoods of nodules of known location (already provided by experts from the ELCAP study). This size of these neighborhoods was selected to be 3 times the template size. For example, with circular templates of radius 10 pixels the neighborhood region is $60 \times 60$ pixels centered at the nodule location marked by the human expert. Figure 39 shows the averaged NCC of the four nodule types overall the corresponding slices (for example on the Juxta-Pleural nodule, we used all the 155 slices having this type of nodule). This figure illustrates the behavior of the NCC measure as a function of distance from the nodule location. The ELCAP expert just identified a point on the nodule not the entire spatial support; this point may not necessarily coincide with the center or the centroid of the nodule. Indeed, the NCC measure decays as we move away from the spatial support of the nodules. Chapter five will show the behavior of the NCC measure using the novel data-driven nodule models; better latching with nodule spatial supports will be illustrated. Even though this is not a focus of this thesis, this information may be used to outline/segment the nodules, in order to conduct further studies subsequent to nodule detection. 


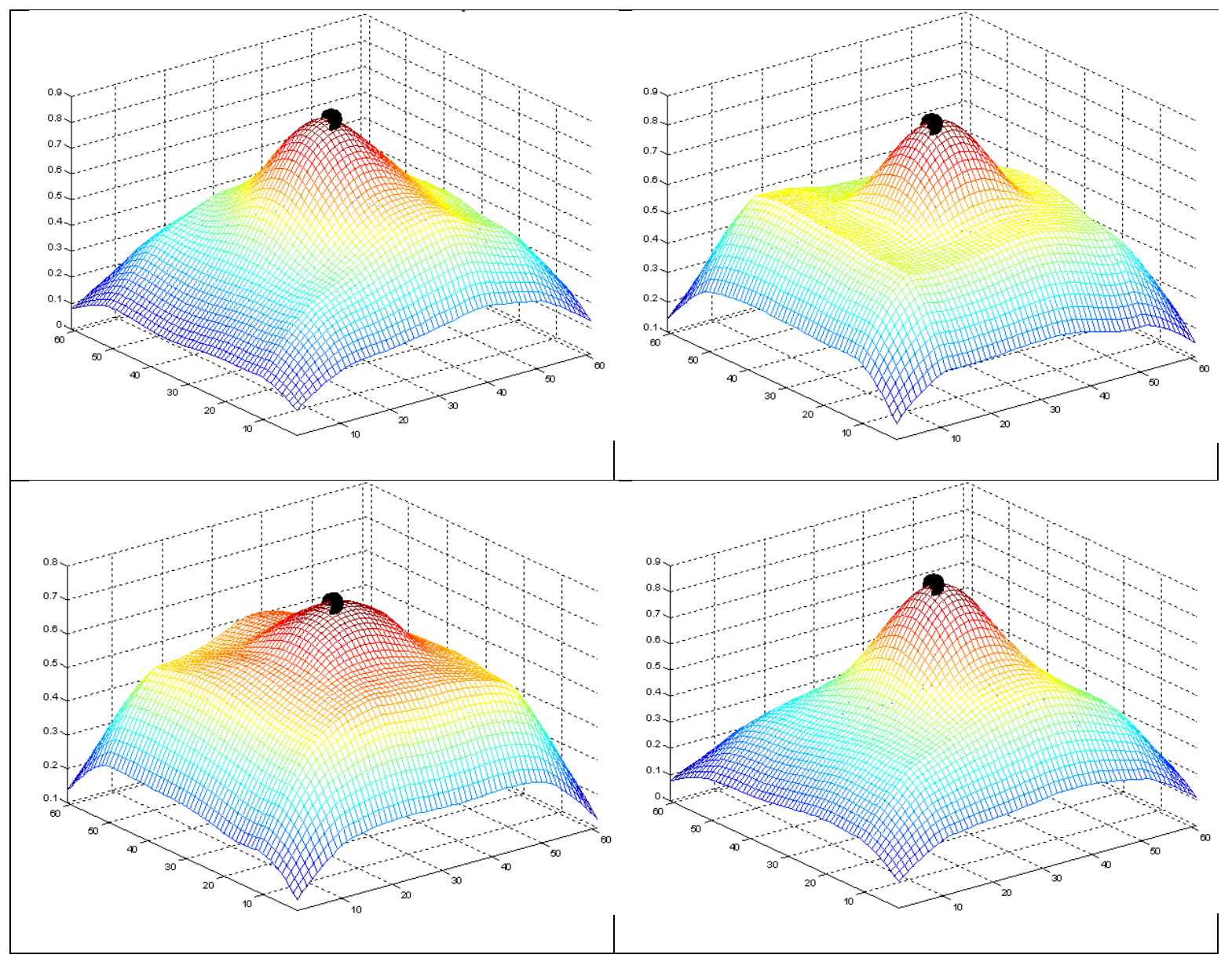

Figure 39: Normalized Cross-Correlation (NCC) for circular templates on regions around the correct nodule location. The figure is the average NCC for all slices having the same nodule type. Upper left is the average NCC for Pleural-Tail nodules, Upper right is the results for Vascular nodules, lower left is the results for Well-Circumscribed nodules, and Lower right shows the results for Juxta-Pleural nodules. The location of the nodules (as provided by the ELCAP experts), shown in black, may not correspond to the center or centroid of the real nodules. 


\section{Sensitivity Analysis of Template Matching:}

The sensitivity of template matching was studied for templates of radii ranging from $0.5 \mathrm{~mm}$ ( 1 pixel) to $10 \mathrm{~mm}$ (20 pixel). The orientations of the templates in the semicircular case are from 0 to 359 with step size of 45 degrees. For each detected nodule, let the coordinates of its centroid be $\mathrm{x}=(\mathrm{x}, \mathrm{y})$, the nodule is considered correctly detected and counted as true positive (TP) when the distance between the detected point $\mathrm{x}$ and the closest ground truth point $\mathrm{g}=(\mathrm{x}, \mathrm{y})$ is smaller than the template radius. All other detected points are considered false positives (FP). True negative $(\mathrm{TN})$ is the number of points which are not detected as candidate nodules and when compared to the ground truth they are not nodules. False negative $(\mathrm{FN})$ is when no point is detected in the neighborhood of the ground truth nodule.

In the following we report the results for template matching using a template of size 10 pixels $(5 \mathrm{~mm})$ and 20 pixels $(10 \mathrm{~mm})$, these results are reported in terms of sensitivity $\mathrm{SN}=\mathrm{TP} /(\mathrm{TP}+\mathrm{FN})$ and specificity $\mathrm{SP}=\mathrm{TN} /(\mathrm{FP}+\mathrm{TN})$.

The sensitivity and specificity are defined in terms of the false positive (FP) and the true positive (TP) nodules. These values are defined with respect to the NCC matching criterion. To generalize the information captured in Figure 39, the behavior of the NCC distribution was studied for various template sizes. Figure 40 shows the NCC distribution for parametric templates of radius 10 pixels (i.e., template size 21x21). As expected, higher values of NCC leads to reduced detection rates, while smaller values increases the detection rates, and consequently the number of FPs. A threshold of NCC value was set 
at 0.5 which have been shown, empirically, to be a good compromise between TP and FPs.

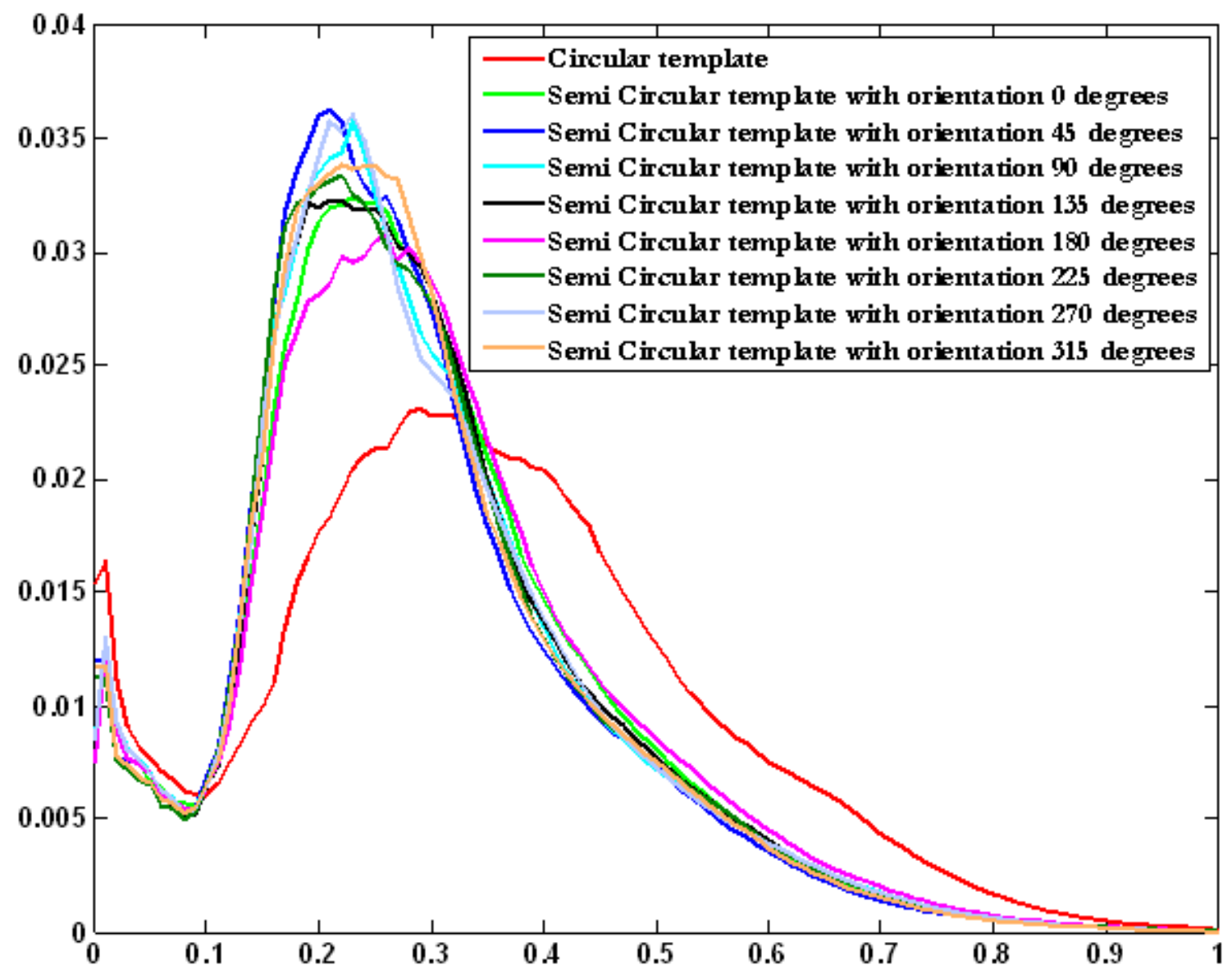

Figure 40: Distribution of the Normalized Cross-Correlation (NCC) for parametric nodules (circular and semi-circular) with radius 10 pixels. Higher NCC values results in less FPs while smaller values provide more FPs. In the ROC plots (Figures 39-42) a NCC threshold of 0.5 was selected.

The receiver operating characteristic (ROC) curve is used to plot the fraction of true positives $(\mathrm{TPR}=$ true positive rate $)$ vs. the fraction of false positives $(\mathrm{FPR}=$ false positive rate $)$, where $\mathrm{TPR}=\mathrm{TP}=(\mathrm{TP}+\mathrm{FN})$ and $\mathrm{FPR}=\mathrm{FP}=(\mathrm{FP}+\mathrm{TN})$. For the sake of 
completeness, we experimented with different template sizes ranging from 1 to 20 pixels $(0.5 \mathrm{~mm}-5 \mathrm{~mm})$.

Figure 41 through 44 show the results for different template shapes for each nodule type. Figure 41 shows that variations in the template shape and/or orientation has minimal affect on well-circumscribed nodule detection.

The semi-circular template with orientation 180 degrees best simulates juxta-pleural as well as pleural-tail, as shown in Figure 42 and 44, while the circular template least represents these nodule types.

These findings for both nodules are justifiable since they are more semi-circular in shape and thus will respond better to such templates. In the case of vascular nodule, the findings were similar to that of the juxta-pleural and pleural-tail nodules but 90 degrees orientation for semi-circular template best represents this nodule type (Figure 43).

Overall, analyzing the effect of template shape for nodule detection on different types showed that variations in the template shape and/or orientation has minimal affect on well-circumscribed nodule detection. The semi-circular template best simulates juxtapleural as well as pleural-tail, while the circular template least represents these nodule types. In the case of vascular nodule, the findings were similar to that of the juxta-pleural and pleural-tail nodules.

Experiments conducted using the template size revealed that smaller template radii yield higher sensitivity, while the performance tends to decrease for larger template sizes. The rate of performance decay depends on template shape and nodule type, the pleural- 
tail nodules are the most sensitive nodule type to the template size, while the vascular nodule can be concluded as the least sensitive to radius template size.

Table 3: Sample results for templates of radius 10

\begin{tabular}{|l|l|l|l|l|l|}
\hline \multicolumn{7}{|c|}{ Circular Template of radius 10 } \\
\hline & $\begin{array}{l}\text { All nodule } \\
\text { types }\end{array}$ & Juxta & Well & Plural Tail & Vascular \\
\hline Sensitivity & $64.6 \%$ & $81.74 \%$ & $31.46 \%$ & $86.96 \%$ & $63.41 \%$ \\
\hline Specificity & $73.7 \%$ & $64.96 \%$ & $79.75 \%$ & $73.89 \%$ & $76.95 \%$ \\
\hline
\end{tabular}

\begin{tabular}{|l|l|l|l|l|l|}
\hline \multicolumn{7}{|c|}{ Semi Circular Template of orientation $0^{\circ}$} \\
\hline & $\begin{array}{l}\text { All nodule } \\
\text { types }\end{array}$ & Juxta & Well & Plural Tail & Vascular \\
\hline Sensitivity & $57.04 \%$ & $76.52 \%$ & $23.6 \%$ & $73.91 \%$ & $56.10 \%$ \\
\hline Specificity & $90.44 \%$ & $86.08 \%$ & $92.73 \%$ & $92.13 \%$ & $92.81 \%$ \\
\hline
\end{tabular}

\begin{tabular}{|l|l|l|l|l|l|}
\hline \multicolumn{7}{|c|}{ Semi Circular Template of orientation 45 } \\
\hline & $\begin{array}{l}\text { All nodule } \\
\text { types }\end{array}$ & Juxta & Well & Plural Tail & Vascular \\
\hline Sensitivity & $57.04 \%$ & $82.61 \%$ & $21.35 \%$ & $69.57 \%$ & $48.78 \%$ \\
\hline Specificity & $91.23 \%$ & $86.92 \%$ & $94.09 \%$ & $93.08 \%$ & $92.28 \%$ \\
\hline
\end{tabular}

\begin{tabular}{|l|l|l|l|l|l|}
\hline \multicolumn{7}{|c|}{ Semi Circular Template of orientation 90 } \\
\hline & $\begin{array}{l}\text { All nodule } \\
\text { types }\end{array}$ & Juxta & Well & Plural Tail & Vascular \\
\hline Sensitivity & $58.42 \%$ & $79.13 \%$ & $26.97 \%$ & $78.26 \%$ & $46.34 \%$ \\
\hline Specificity & $89.57 \%$ & $83.68 \%$ & $92.9 \%$ & $91.56 \%$ & 92.56 \\
\hline
\end{tabular}

\begin{tabular}{|l|l|l|l|l|l|}
\hline \multicolumn{7}{|c|}{ Semi Circular Template of orientation 135 } \\
\hline & $\begin{array}{l}\text { All nodule } \\
\text { types }\end{array}$ & Juxta & Well & Plural Tail & Vascular \\
\hline Sensitivity & $57.73 \%$ & $77.39 \%$ & $24.72 \%$ & $73.91 \%$ & $56.1 \%$ \\
\hline Specificity & $90.32 \%$ & $84.98 \%$ & $93.63 \%$ & $91.30 \%$ & $92.73 \%$ \\
\hline
\end{tabular}


Table 4: Sample results for templates of radius 20

\begin{tabular}{|l|l|l|l|l|l|}
\hline \multicolumn{7}{|c|}{ Circular Template of radius 20 } \\
\hline & $\begin{array}{l}\text { All nodule } \\
\text { types }\end{array}$ & Juxta & Well & Plural Tail & Vascular \\
\hline Sensitivity & $71.48 \%$ & $83.48 \%$ & $46.07 \%$ & $89.13 \%$ & $73.17 \%$ \\
\hline Specificity & $55.48 \%$ & $54.6 \%$ & $61.06 \%$ & $46.29 \%$ & $54.24 \%$ \\
\hline
\end{tabular}

\begin{tabular}{|l|l|l|l|l|l|}
\hline \multicolumn{7}{|c|}{ Semi Circular Template of orientation $0^{\circ}$} \\
\hline & $\begin{array}{l}\text { All nodule } \\
\text { types }\end{array}$ & Juxta & Well & Plural Tail & Vascular \\
\hline Sensitivity & $57.39 \%$ & $69.57 \%$ & $33.71 \%$ & $71.74 \%$ & $58.54 \%$ \\
\hline Specificity & $68.77 \%$ & $61.43 \%$ & $74.94 \%$ & $69.09 \%$ & $71.43 \%$ \\
\hline
\end{tabular}

\begin{tabular}{|l|l|l|l|l|l|}
\hline \multicolumn{7}{|c|}{ Semi Circular Template of orientation $45^{\circ}$} \\
\hline & $\begin{array}{l}\text { All nodule } \\
\text { types }\end{array}$ & Juxta & Well & Plural Tail & Vascular \\
\hline Sensitivity & $58.08 \%$ & $73.04 \%$ & $29.21 \%$ & $73.91 \%$ & $60.98 \%$ \\
\hline Specificity & $70.73 \%$ & $66.44 \%$ & $75.97 \%$ & $68.3 \%$ & $72.01 \%$ \\
\hline
\end{tabular}

\begin{tabular}{|l|l|l|l|l|l|}
\hline \multicolumn{7}{|c|}{ Semi Circular Template of orientation 90 } \\
\hline & $\begin{array}{l}\text { All nodule } \\
\text { types }\end{array}$ & Juxta & Well & Plural Tail & Vascular \\
\hline Sensitivity & $60.82 \%$ & $78.26 \%$ & $31.46 \%$ & $73.91 \%$ & $60.98 \%$ \\
\hline Specificity & $66.64 \%$ & $62.64 \%$ & $69.83 \%$ & $64.48 \%$ & $70.68 \%$ \\
\hline
\end{tabular}

\begin{tabular}{|l|l|l|l|l|l|}
\hline \multicolumn{7}{|c|}{ Semi Circular Template of orientation $135^{\circ}$} \\
\hline & $\begin{array}{l}\text { All nodule } \\
\text { types }\end{array}$ & Juxta & Well & Plural Tail & Vascular \\
\hline Sensitivity & $60.82 \%$ & $76.52 \%$ & $34.83 \%$ & $67.39 \%$ & $65.85 \%$ \\
\hline Specificity & $66.84 \%$ & $61.27 \%$ & $72.33 \%$ & $63.64 \%$ & $70.10 \%$ \\
\hline
\end{tabular}

\begin{tabular}{|l|l|l|l|l|l|}
\hline \multicolumn{7}{|c|}{ Semi Circular Template of orientation $180^{\circ}$} \\
\hline & $\begin{array}{l}\text { All nodule } \\
\text { types }\end{array}$ & Juxta & Well & Plural Tail & Vascular \\
\hline Sensitivity & $61.86 \%$ & $73.04 \%$ & $37.08 \%$ & $73.91 \%$ & $70.73 \%$ \\
\hline Specificity & $64.79 \%$ & $60.35 \%$ & $69.48 \%$ & $62.02 \%$ & $68.08 \%$ \\
\hline
\end{tabular}

\begin{tabular}{|l|l|l|l|l|l|}
\hline \multicolumn{7}{|c|}{ Semi Circular Template of orientation 225 } \\
\hline & $\begin{array}{l}\text { All nodule } \\
\text { types }\end{array}$ & Juxta & Well & Plural Tail & Vascular \\
\hline Sensitivity & $61.17 \%$ & $77.39 \%$ & $30.34 \%$ & $76.09 \%$ & $65.85 \%$ \\
\hline Specificity & $66.51 \%$ & $60.95 \%$ & $71.48 \%$ & $65.4 \%$ & $69.61 \%$ \\
\hline
\end{tabular}


ROC for different templates applied over well circumscribed nodules

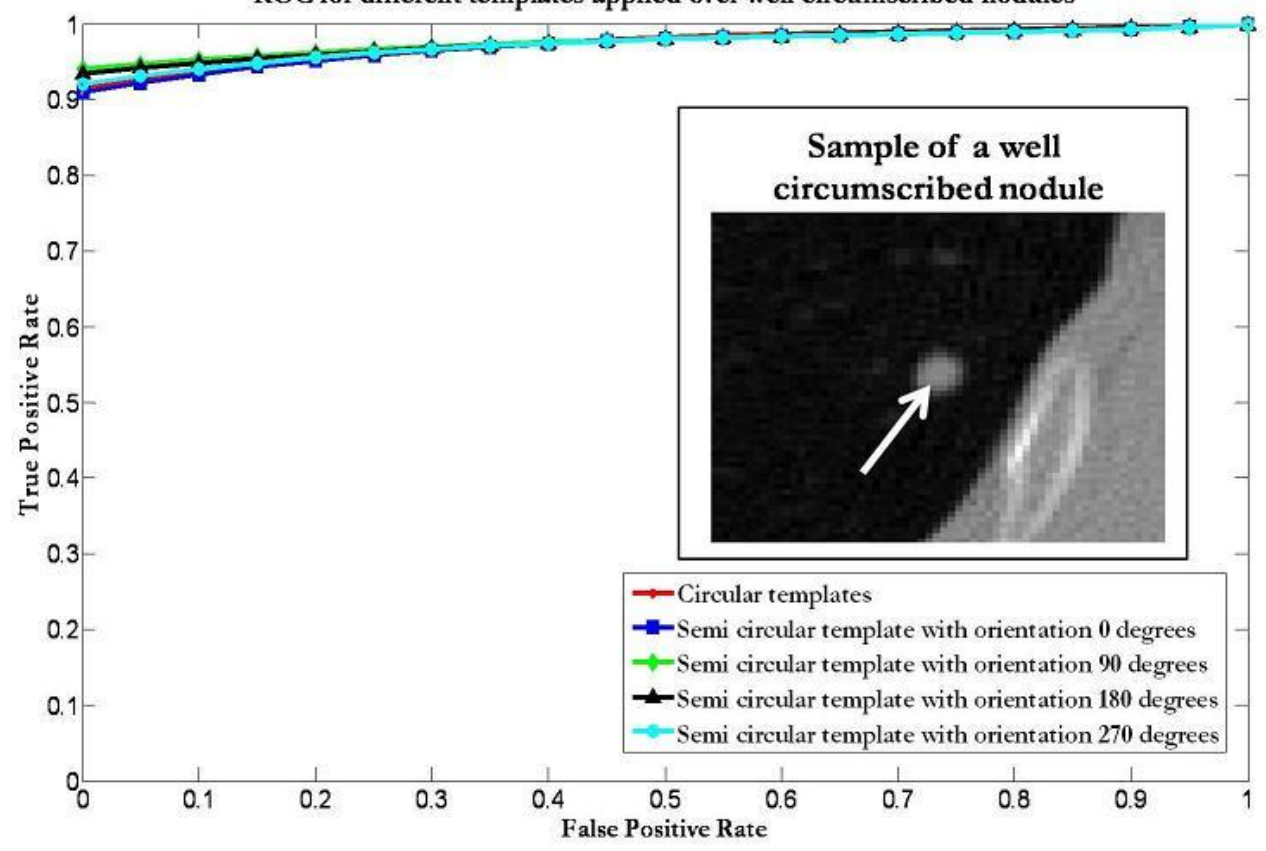

Figure 41: ROC curve of different template shape applied over well circumscribed nodules

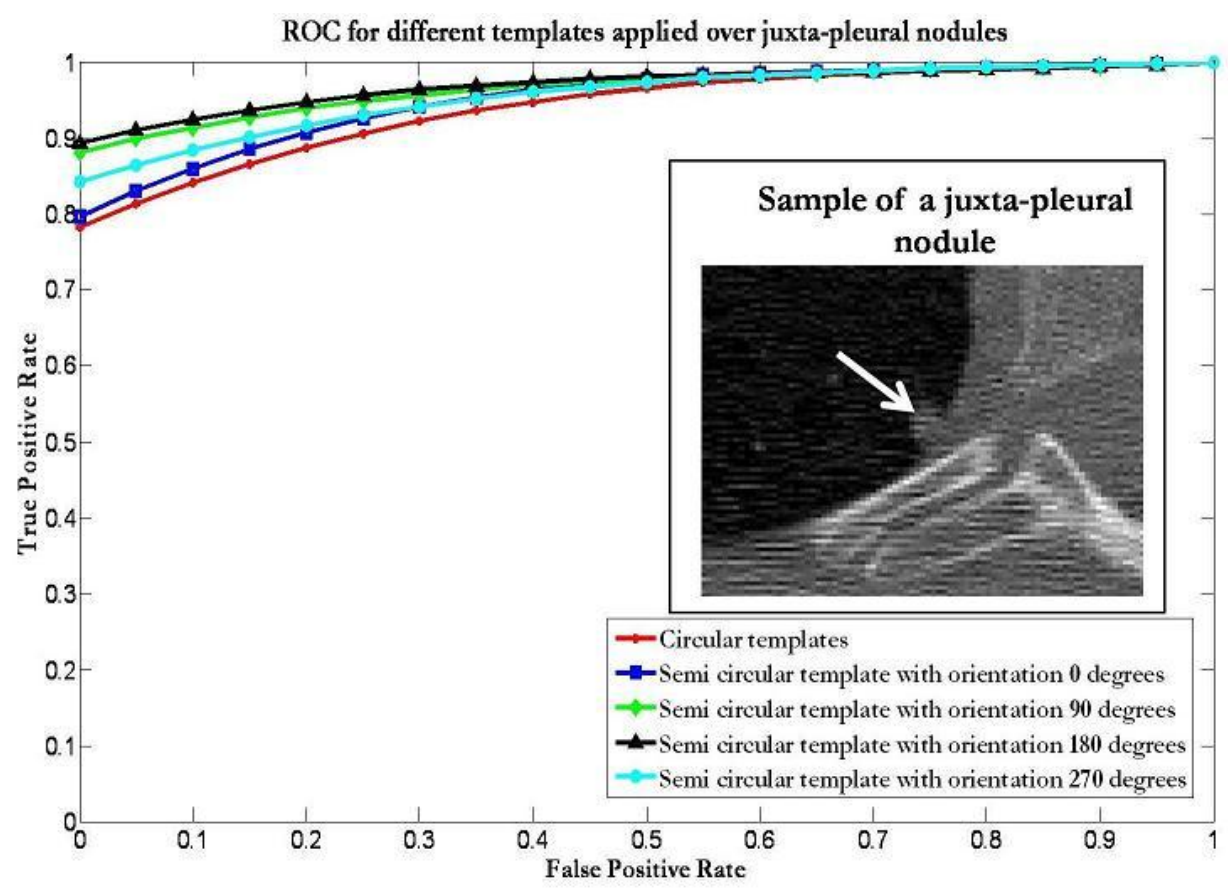

Figure 42: ROC curve of different template shape applied over juxta-pleural nodules 


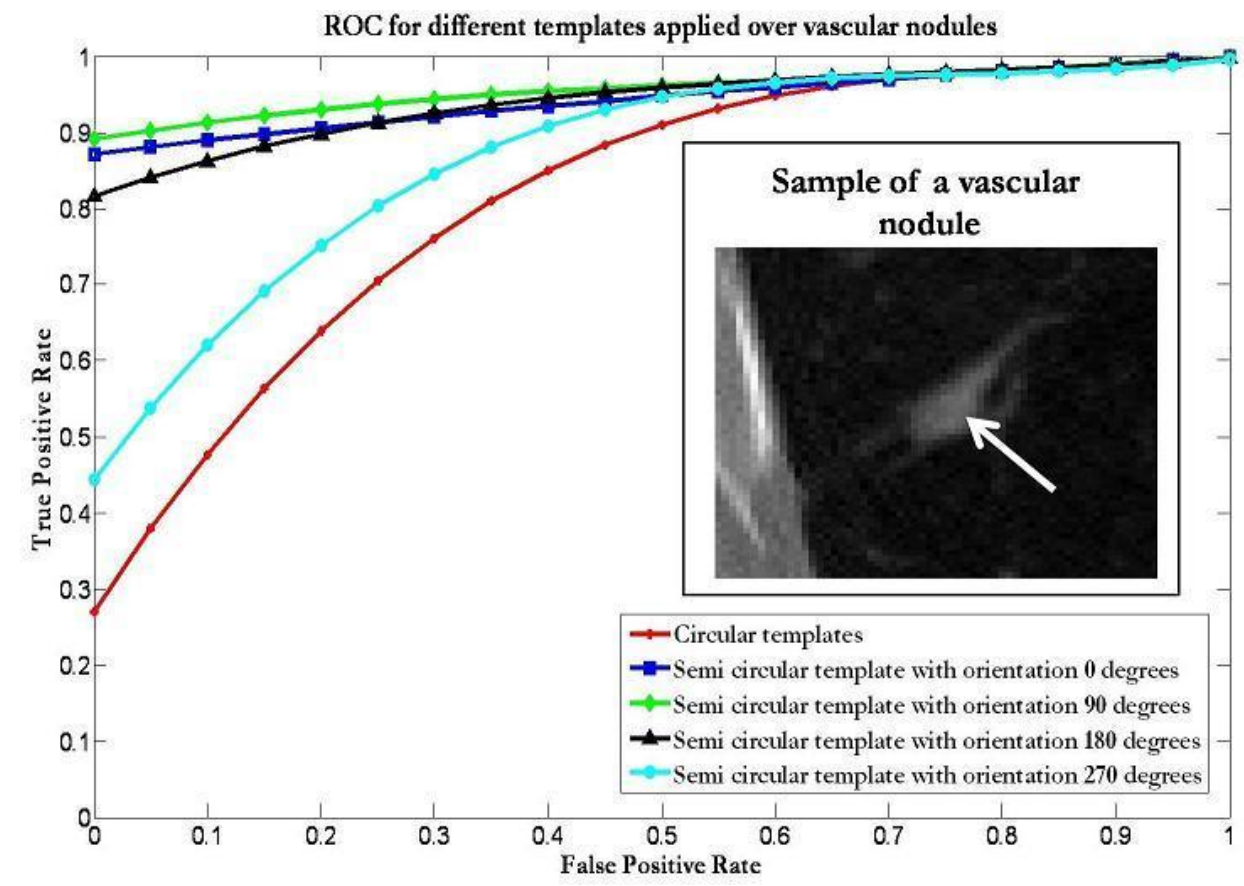

Figure 43: ROC curve of different template shape applied over vascular nodules.

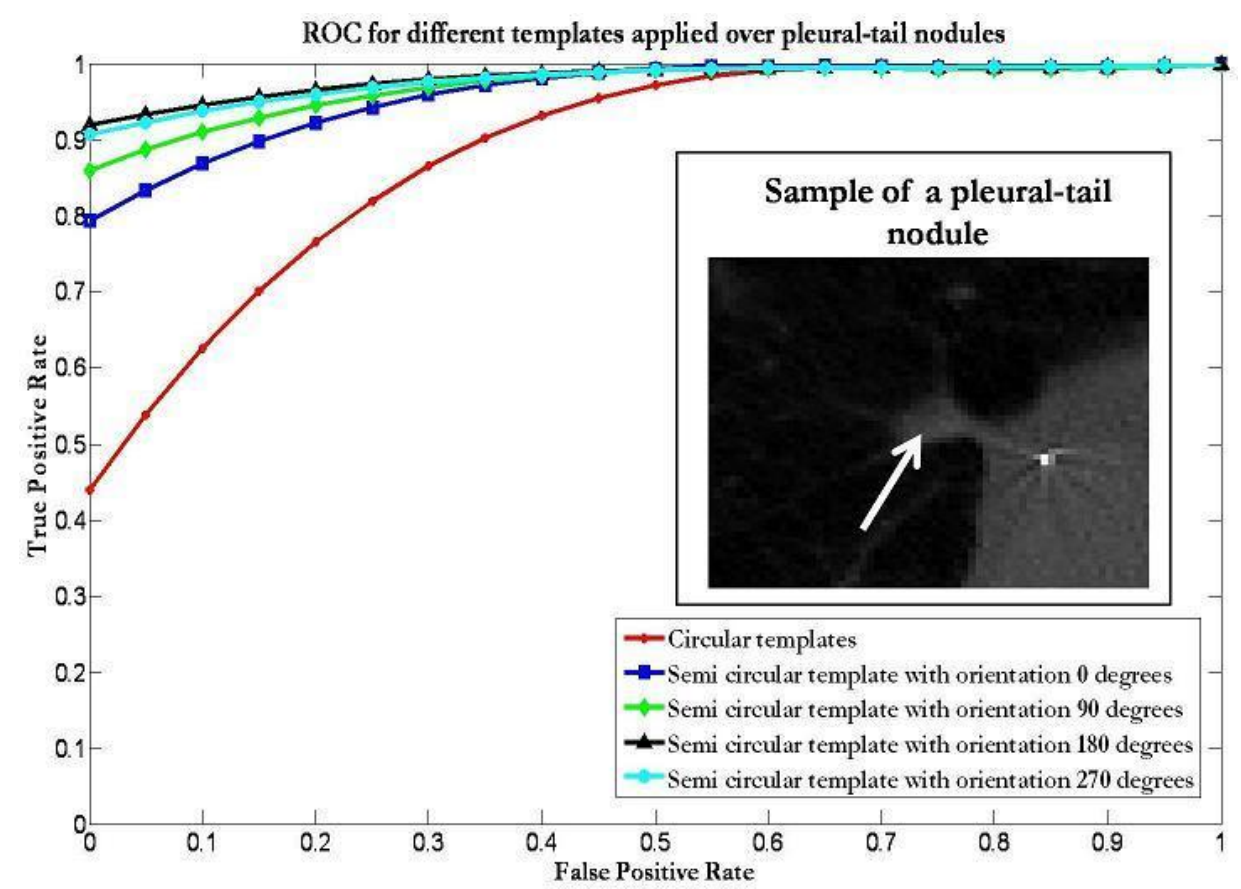

Figure 44: ROC curve of different template shape applied over pleural-tail nodules. 


\section{F.SUMMARY}

This chapter reports improved results for automatic detection and classification of

lung nodules using low dose CT (LDCT) scans. The approach uses parametric templates for nodule detection. The chapter describes an approach for template design and nodule detection. The chapter also investigated the sensitivity of template matching using the ELCAP lung database. 


\section{CHAPTER 5}

\section{NOVEL APPROACH FOR NODULE MODELING}

\section{$\underline{\text { A. Introduction }}$}

This chapter describes a novel approach for nodule detection that may lead to a robust performance of the template matching process. The idea is to use the data to design the template. From a training dataset of nodules of various types we generate an intelligent template, per nodule, that contains texture and the basic shape of the nodule. This intelligent template will be used instead of the synthetic templates (e.g., the parametric templates studied in Chapter 4) in the nodule detection process. Template matching, for nodule detection, using the data-driven templates should provide better performance than the synthetic templates. Eventually, intelligence may be added to discriminate between various pathologies as well. Below we describe the basic components of this approach and preliminary evaluations.

\section{B. Data-Driven Nodule Modeling}

Starting from pre-identified nodules, we employed novel shape analysis approaches to create a new template model that captures the shape and textural information of each nodule type. These new models are used for detection as well as classification of lung nodules shown in LDCT scans.

Note: A patent application with the University of Louisville, Office of Technology Transfer is in progress based on the material covered in this chapter-May 2009. 
Based on our analysis in Chapter 4, we chose the bounding box around the location of the nodule to be of size $21 \times 21$ pixels (this corresponds to spatial resolution of $1.5 \mathrm{~cm} \mathrm{x}$ $1.5 \mathrm{~cm}$; thus covering the basic nodule sizes expected in LDCT scans). The ensemble of nodules contains variations in intensity distribution, shape/structural information and directional variability. The cropped regions inside the bounding boxes will maintain such variations. Co-registering these regions with respect to structures/shapes will generate another dataset, which when averaged will create an average nodule that conveys the statistics of the ensemble. This average nodule will be the "intelligent template" which will be used in the template matching process. We will generate one such template per nodule type. Various statistical methods may be employed in the future in building the template process, including Principle Component Analysis (PCA), active appearance modeling, and indeed Spherical Harmonics (e.g., [61][62]). Co-registration may be performed using normalized mutual information (NMI) approach or similar methods; the features for co-registration are the basic shape information of each nodule.

The procedure to generate a smart nodule model is as follows:

- First we select an ensemble of nodules (in our implementation using the ELCAP data we used 24 samples from each nodule type) to generate a template per nodule type.

- Then we annotate those nodules to highlight the basic geometric and structural features of the nodules.

- We use Procrustes analysis to co-register those nodules with respect to any member of the ensemble, e.g., we may use the first nodule as reference. 
- The mean of the co-registered nodules will be the template which will be used in subsequent analysis steps.

Figure 45 summarizes the process of generating the new template model.

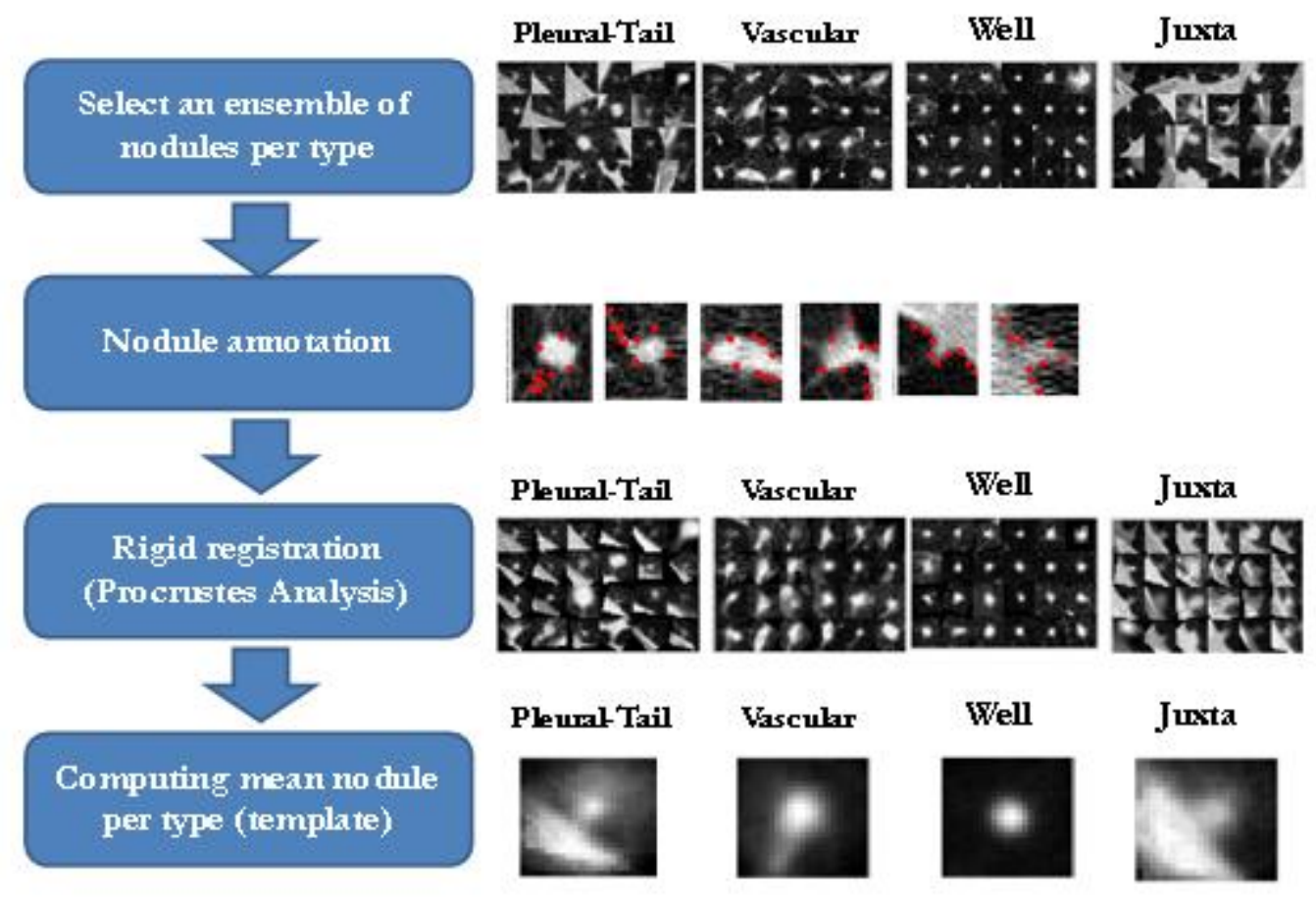

Figure 45: Procedure to generate data-driven nodule model.

\section{Procrustes approach [63-64]}

Figure 46 illustrates the Procrustes distance between two shapes $a$ and $b$. There are several preprocessing steps to determine the Procrustes distance between two shapes [68]:

1. Compute the centroid of each shape.

2. Rescale each shape to have equal size.

3. Align with respect to position the two shapes at their centroids. 
4. Align with respect to orientation by rotation.
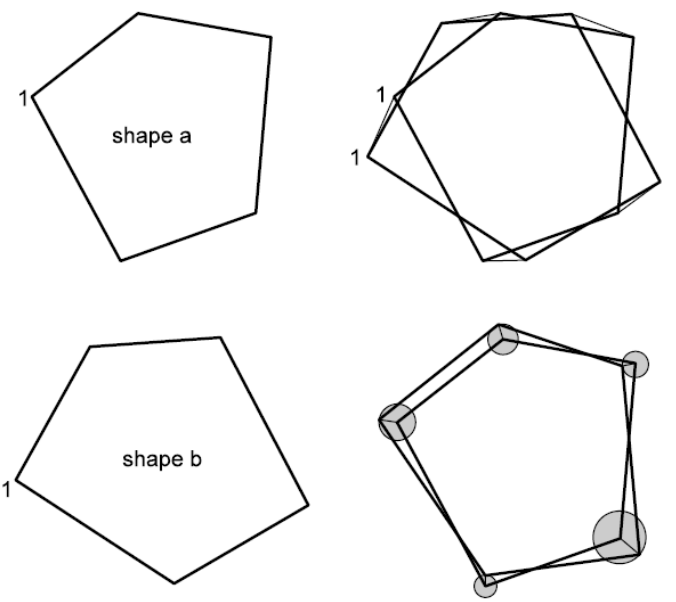

Figure 46: Procrustes distance between two shapes a and .

The Procrustes distance $\left(M^{2}\right)$ between two shapes $x=\left(x_{x i}, x_{y i}\right)$ and $y=\left(y_{x i}, y_{y i}\right)$ is a least-squares type of metric of the form:

$$
M^{2}=\|x-y\|^{2}
$$

Procrustes distance-based rigid registration between two shapes usually involves minimizing the expression $\|T(x)-y\|^{2}$, where $T$ in the Euclidean case is

$$
T\left(\begin{array}{l}
x \\
y
\end{array}\right)=\left[\begin{array}{cc}
a & -b \\
b & a
\end{array}\right]\left[\begin{array}{l}
x \\
y
\end{array}\right]+\left[\begin{array}{l}
t_{x} \\
t_{y}
\end{array}\right]
$$

The term $\|T(x)-y\|^{2}$ can simply be differentiated with respect to the parameters $a, b, t_{x}$ and $t_{y}$, to determine the optimum parameters that minimize the expression $\|T(x)-y\|^{2}$. The actual closed-form solutions of the parameters are: 


$$
\begin{aligned}
& a=(x \cdot y) /|x|^{2}, \quad b=\left(\sum_{i} x_{x i} y_{y i}-x_{y i} y_{x i}\right) /|x|^{2} \\
& \left.t_{x}=(1 / n) \sum_{i} y_{x i}, \quad t_{y}=(1 / n) \sum_{i} y_{y i}\right)
\end{aligned}
$$

In [69], one can find detailed proofs of the above parameters.

\section{Shape Warping}

Suppose we wish to warp a 2D shape $s=\left(x_{1}, y_{1}, \cdots, x_{n}, y_{n}\right)^{T}$, so that the set of $n$ control

points $\boldsymbol{x}_{\boldsymbol{i}}=\left\{x_{i}, y_{i}\right\}$ are mapped to new positions, $\boldsymbol{x}_{\boldsymbol{i}}^{\prime}=\left\{x_{i}^{\prime}, y_{i}^{\prime}\right\}$. We require a continuous vector-valued mapping function, $\boldsymbol{f}$, such that $\boldsymbol{f}\left(\boldsymbol{x}_{\boldsymbol{i}}\right)=\boldsymbol{x}_{\boldsymbol{i}}^{\prime}$. This function is a type of forward-mapping method. One such transformation is

$$
\left[\begin{array}{l}
x_{i}^{\prime} \\
y_{i}^{\prime}
\end{array}\right]=s\left[\begin{array}{ll}
a & b \\
c & d
\end{array}\right]\left[\begin{array}{l}
x_{i} \\
y_{i}
\end{array}\right]+\left[\begin{array}{l}
t_{x} \\
t_{y}
\end{array}\right]
$$

The Matlab built-in function procrustes [70] solves for the parameterss, $a, b, c, d, t_{x}$, and $t_{y}$, given the corresponding pair of control points $\boldsymbol{x}_{\boldsymbol{i}}=\left\{x_{i}, y_{i}\right\}$ and $\boldsymbol{x}_{\boldsymbol{i}}^{\prime}=\left\{x_{i}^{\prime}, y_{i}^{\prime}\right\}$.

For example, to warp Shape 1 (with control points $\boldsymbol{x}_{\boldsymbol{i}}$ ) to Shape 2 (with control points $\boldsymbol{x}_{\boldsymbol{i}}^{\prime}$ ) in Figure 47, the numerical values for the control points are

$$
\begin{aligned}
& \boldsymbol{x}_{\boldsymbol{i}}=\left(x_{1}, y_{1}, x_{2}, y_{2}, x_{3}, y_{3}\right)=(0.0,1.0,1.0,1.0,0.5,0.0)^{T} \text { and } \\
& \boldsymbol{x}_{\boldsymbol{i}}^{\prime}=(2,2.5,2.5,2.5,2.25,2)^{T}
\end{aligned}
$$




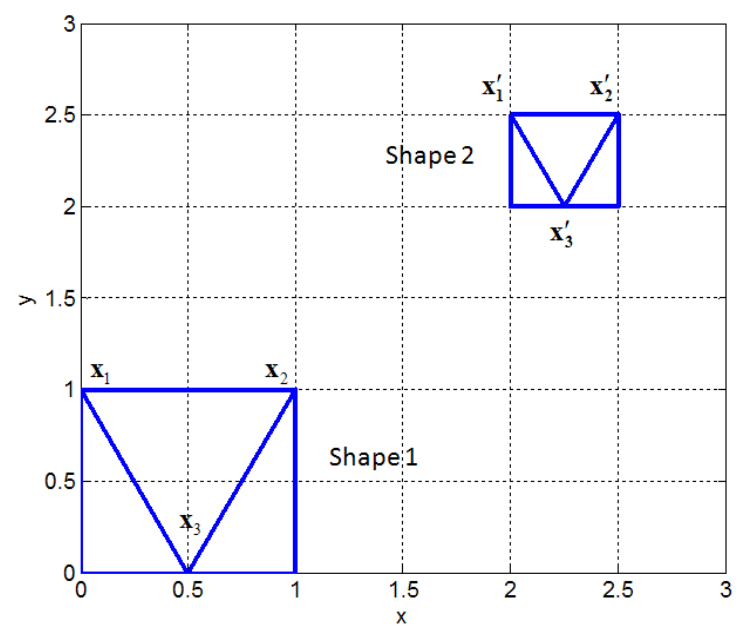

Figure 47: Two shapes (together with the control points) used for the shape warping numerical example.

Using the procrustes (e.g., procrustes $\left.\left(\boldsymbol{x}_{\boldsymbol{i}}^{\prime}, \boldsymbol{x}_{\boldsymbol{i}}\right)\right)$ function, the resulting parameters (using the corresponding control points) are

$$
s=0.5,\left[\begin{array}{ll}
a & b \\
c & d
\end{array}\right]=\left[\begin{array}{ll}
1 & 0 \\
0 & 1
\end{array}\right],\left[\begin{array}{l}
t_{x} \\
t_{y}
\end{array}\right]=\left[\begin{array}{l}
2 \\
2
\end{array}\right]
$$

The new vertices of Shape 1 ( $x_{i}$ - first column, $y_{i}$ - second column) after warping, are the following (and notice that it corresponds to Shape 2 in Figure 47),

$$
\left[\begin{array}{cc}
2 & 2.5 \\
2.5 & 2.5 \\
2.25 & 2 \\
2 & 2 \\
2.5 & 2
\end{array}\right]=0.5 *\left[\begin{array}{cc}
0 & 1 \\
1 & 1 \\
0.5 & 0 \\
0 & 0 \\
1 & 0
\end{array}\right]\left[\begin{array}{ll}
1 & 0 \\
0 & 1
\end{array}\right]+\left[\begin{array}{ll}
2 & 2 \\
2 & 2 \\
2 & 2 \\
2 & 2 \\
2 & 2
\end{array}\right]
$$

\section{Image Warping}

Suppose we wish to warp an image $\boldsymbol{I}$ so that the set of $n$ control points $\boldsymbol{x}_{\boldsymbol{i}}=$ $\left\{x_{i}, y_{i}\right\}$ are mapped to new positions, $\boldsymbol{x}_{\boldsymbol{i}}^{\prime}=\left\{x_{i}^{\prime}, y_{i}^{\prime}\right\}$. We again require a continuous vector- 
valued mapping function, $\boldsymbol{f}$, such that $\boldsymbol{f}\left(\boldsymbol{x}_{\boldsymbol{i}}\right)=\boldsymbol{x}_{\boldsymbol{i}}^{\prime}$. Given such a function, we can project each pixel of image $\boldsymbol{I}$ to a new image $\boldsymbol{I}^{\prime}$. However, in practice, it is better to find the reverse map $\boldsymbol{f}^{\prime}$ (reverse-mapping), taking the control points $\boldsymbol{x}_{\boldsymbol{i}}^{\prime}$ to $\boldsymbol{x}_{\boldsymbol{i}}$, in order to avoid interpolation problems. For each pixel in the target warped image $\boldsymbol{I}^{\prime}$, we can determine where it came from $\boldsymbol{I}$ and fill it in.

Figure 48 shows the difficulty of implementing an image warping forward-map. After iterating over the source image, two problems may occur: (a) many source pixels can map to the same destination pixel and (b) some destination pixels may not be even covered.

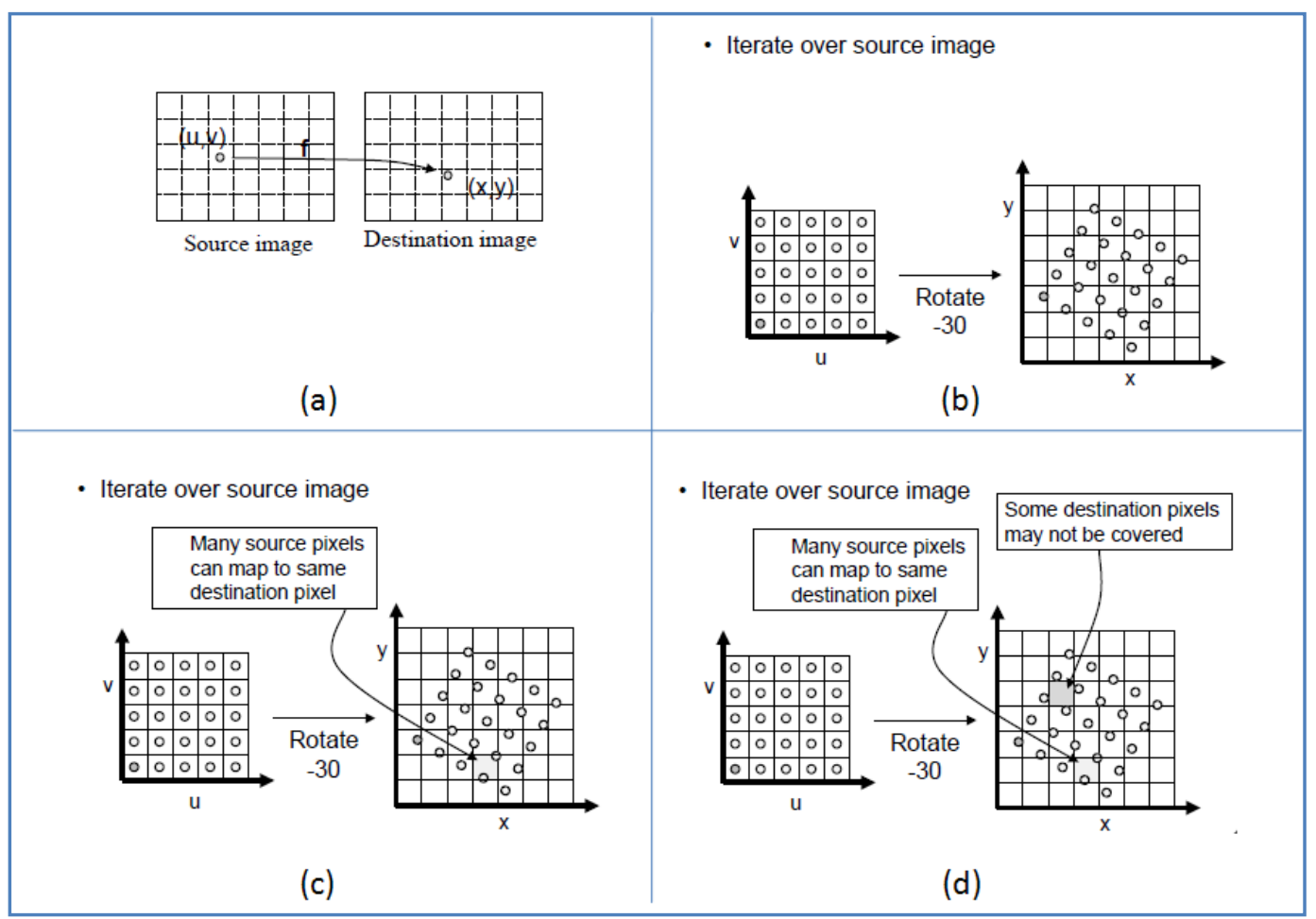

Figure 48: Forward-map image warping. (a) Source (containing $\boldsymbol{x}_{\boldsymbol{i}}$ ) and destination (containing $\left.\boldsymbol{x}_{\boldsymbol{i}}^{\prime}\right)$ images. $\left(u_{i}, v_{i}\right)$ are integer values and $\left(x_{i}, y_{i}\right)$ are real values. (b) Apply forward-map $f$ to all source image pixels. (c) Many source pixels can map to the same destination pixel. (d) Some destination pixels may not be even covered. 
Figure 49 , on the other hand, illustrates the practicality of implementing a reverse-map image warping. Since iteration over the destination image is performed, there is no possibility that the problems existing with the forward-map warping, will occur.

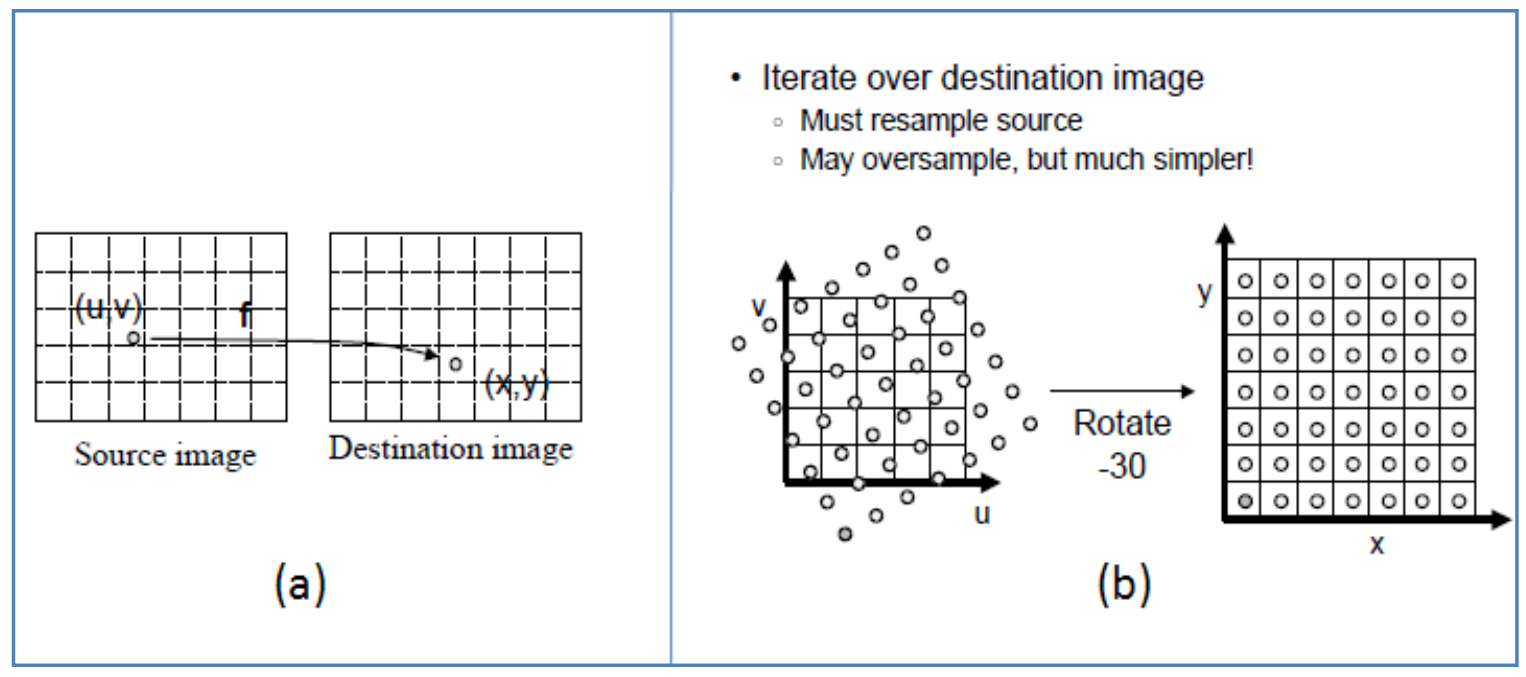

Figure 49: Reverse-map image warping. (a) Source (containing $\boldsymbol{x}_{\boldsymbol{i}}$ ) and destination (containing $\left.\boldsymbol{x}_{\boldsymbol{i}}^{\prime}\right)$ images. $\left(u_{i}, v_{i}\right)$ are real values and $\left(x_{i}, y_{i}\right)$ are integer values. $(b)$ Iterate over the destination image. It is necessary to resample source (e.g., bilinear, cubic interpolation). Notice that all pixels in the destination image can be easily determined after resampling, unlike in Figure 48.

Numerical Example: Figure 50 shows the source (a) and destination (b) images, for this example. The goal is to warp the source image (with control points $\boldsymbol{x}_{\boldsymbol{i}}$ ) to the destination image (with control points $\boldsymbol{x}_{\boldsymbol{i}}^{\prime}$ ). The forward-map method finds the function $\boldsymbol{f}$ that maps the points $\boldsymbol{x}_{\boldsymbol{i}}$ to $\boldsymbol{x}_{\boldsymbol{i}}^{\prime}$. From the previous paragraphs, the reverse-map approach is better suited for image warping, i.e., find the function $\boldsymbol{f}^{\prime}$ that maps the points $\boldsymbol{x}_{\boldsymbol{i}}^{\prime}$ to $\boldsymbol{x}_{\boldsymbol{i}}$. The final warped images is shown in (c). 


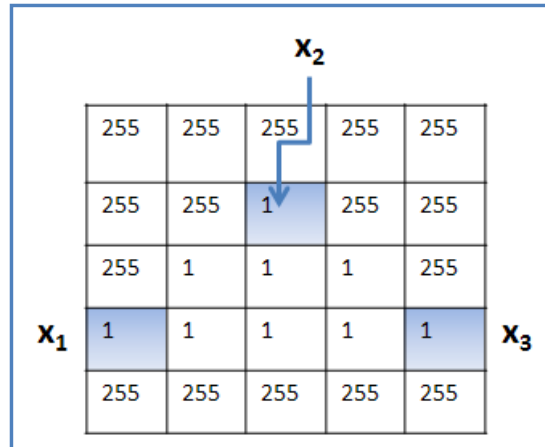

(a)

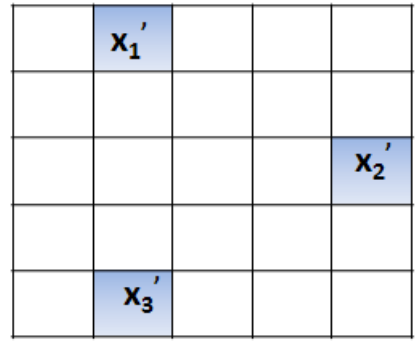

(b)

\begin{tabular}{|l|l|l|l|l|}
\hline 255 & 49.4 & 122 & 220 & 255 \\
\hline 255 & 49.4 & 1 & 115 & 233 \\
\hline 255 & 49.4 & 1 & 1 & 98 \\
\hline 255 & 49.4 & 1 & 115 & 233 \\
\hline 255 & 49.4 & 122 & 220 & 255 \\
\hline
\end{tabular}

(c)

Figure 50: Image warping numerical example illustration. (a) Source image with pixel values and control points. (b) Destination image with unfilled pixel values and control points. (c) The actual destination image after warping the source image, according to the control points.

\section{Implementations:}

As stated above, a sample of 24 nodules were used from each nodule type using the ELCAP data. The nodules were co-registered with respect to the first member of the ensemble using the above approach. The annotation was performed manually, using the main characteristics of each nodule type. These main characteristics identify the landmarks (correspondence points) for each member of the ensemble in order to perform the registration process. More landmarks may be generated to provide a smooth representation of the nodule contour which can be used by itself for shape analysis and other intrinsic properties of the particular nodule type.

Figures 51-54 show the results of co-registering the ensembles of the four nodule types. Important to notice the effect of co-registration on the average nodule shape as compared to the average without registration. 


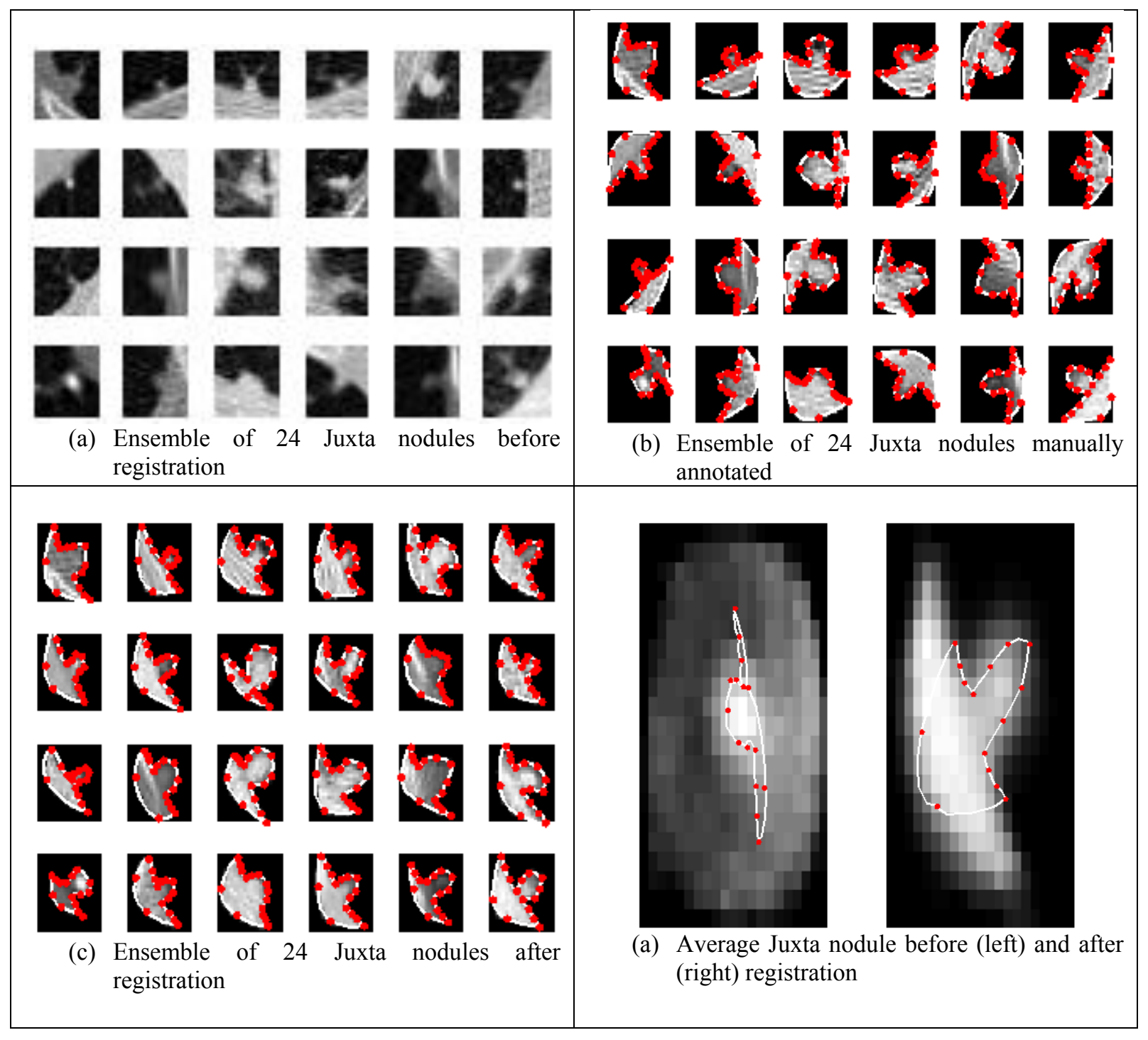

Figure 51: Generating the Juxta-Pleural nodule. (a) Original (non registered nodules); (b) manually annotated nodules; (c) co-registered (with respect to first nodule) nodules; and $(d)$ average nodule with and without registration. Notice the averaging process creates a nodule which maintains the main characteristics of Juxta-Pleural nodules. 


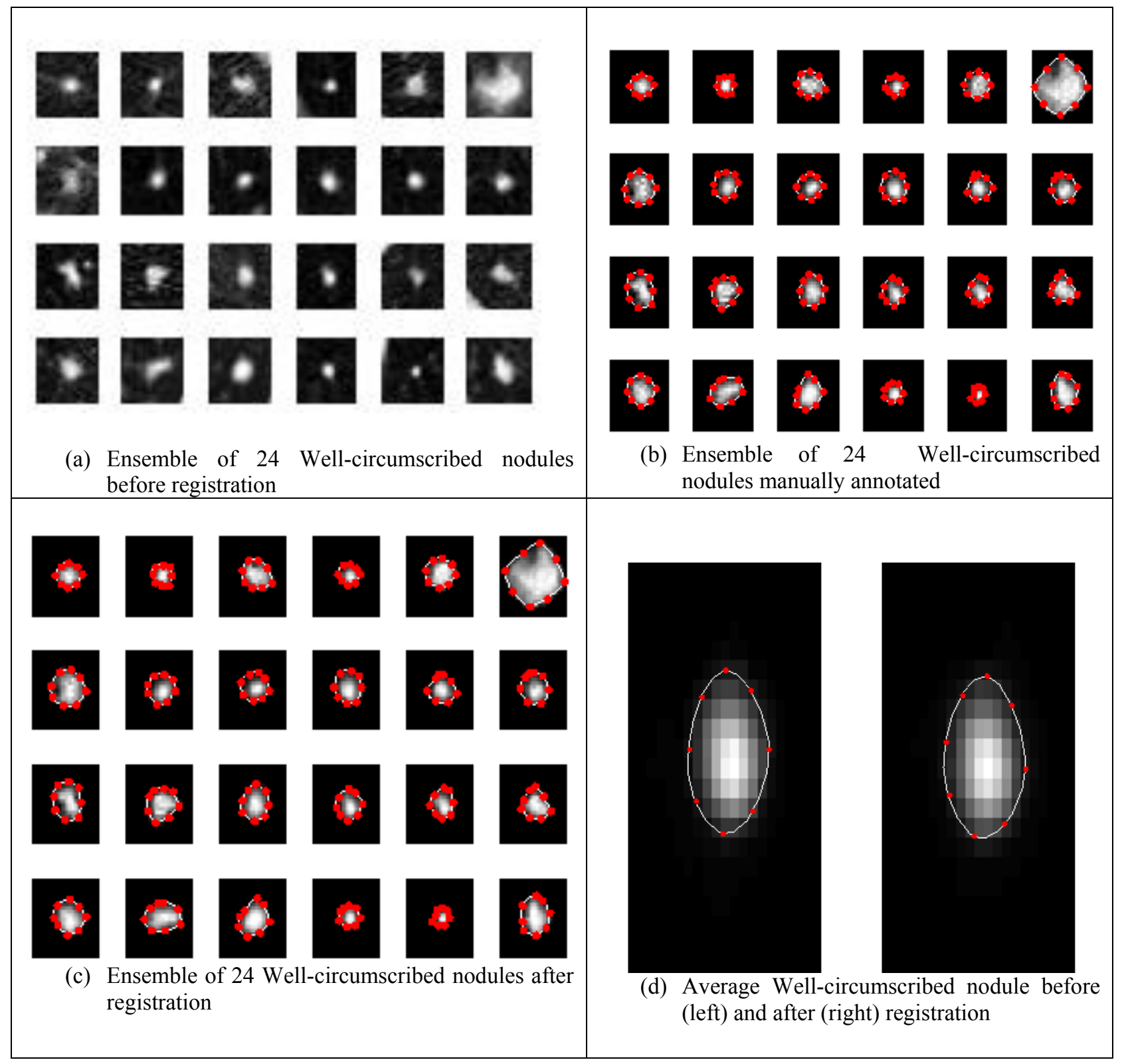

Figure 52: Generating the Well-Circumscribed nodule. (a) Original (non registered nodules); (b) manually annotated nodules; (c) co-registered (with respect to first nodule) nodules; and $(d)$ average nodule with and without registration. Notice the averaging process creates a nodule which maintains the main characteristics of Well-

Circumscribed nodules. 


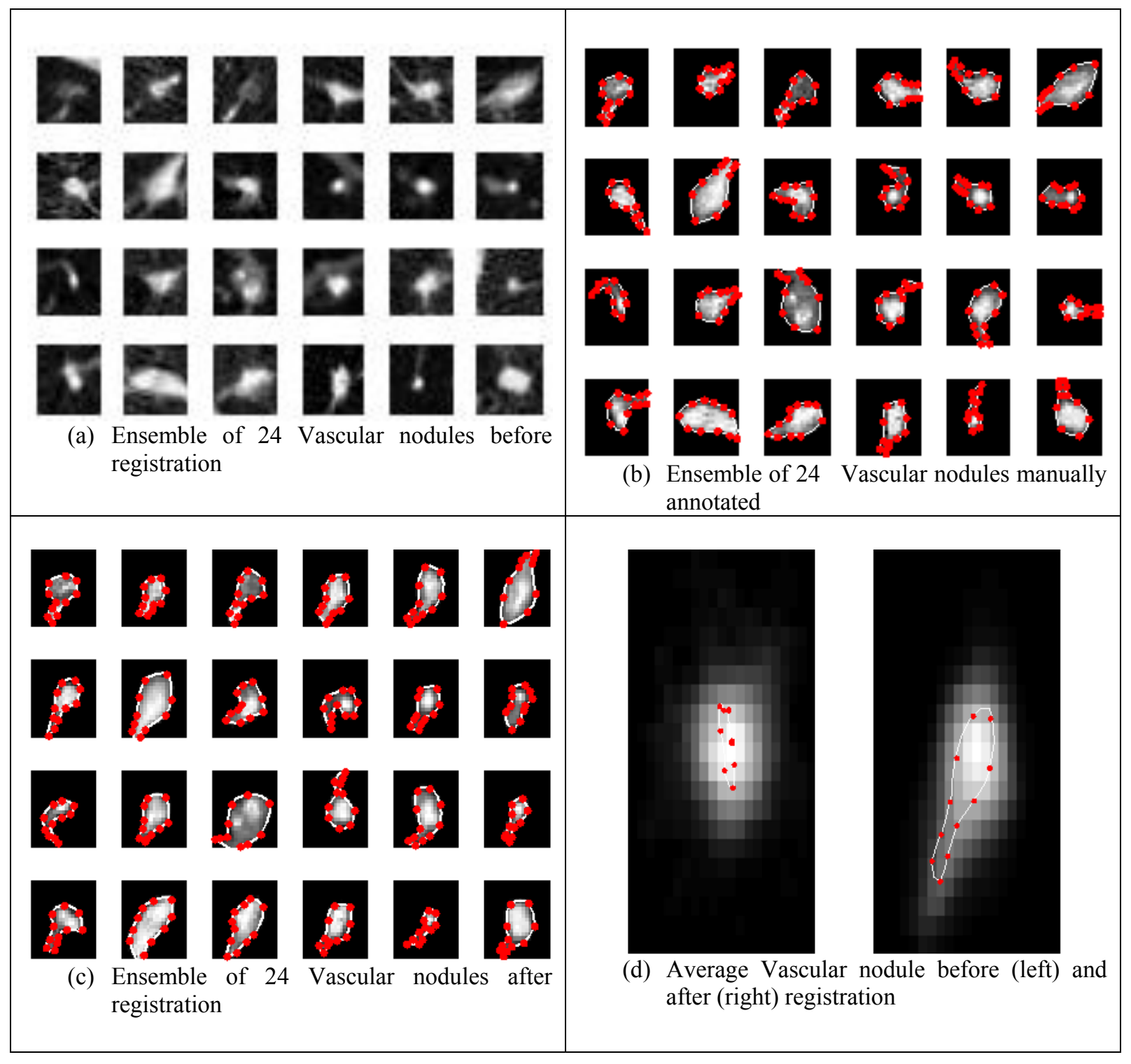

Figure 53: Generating the Vascular nodule. (a) Original (non registered nodules); (b) manually annotated nodules; (c) co-registered (with respect to first nodule) nodules; and (d) average nodule with and without registration. Notice the averaging process creates a nodule which maintains the main characteristics of Vascular nodules. 


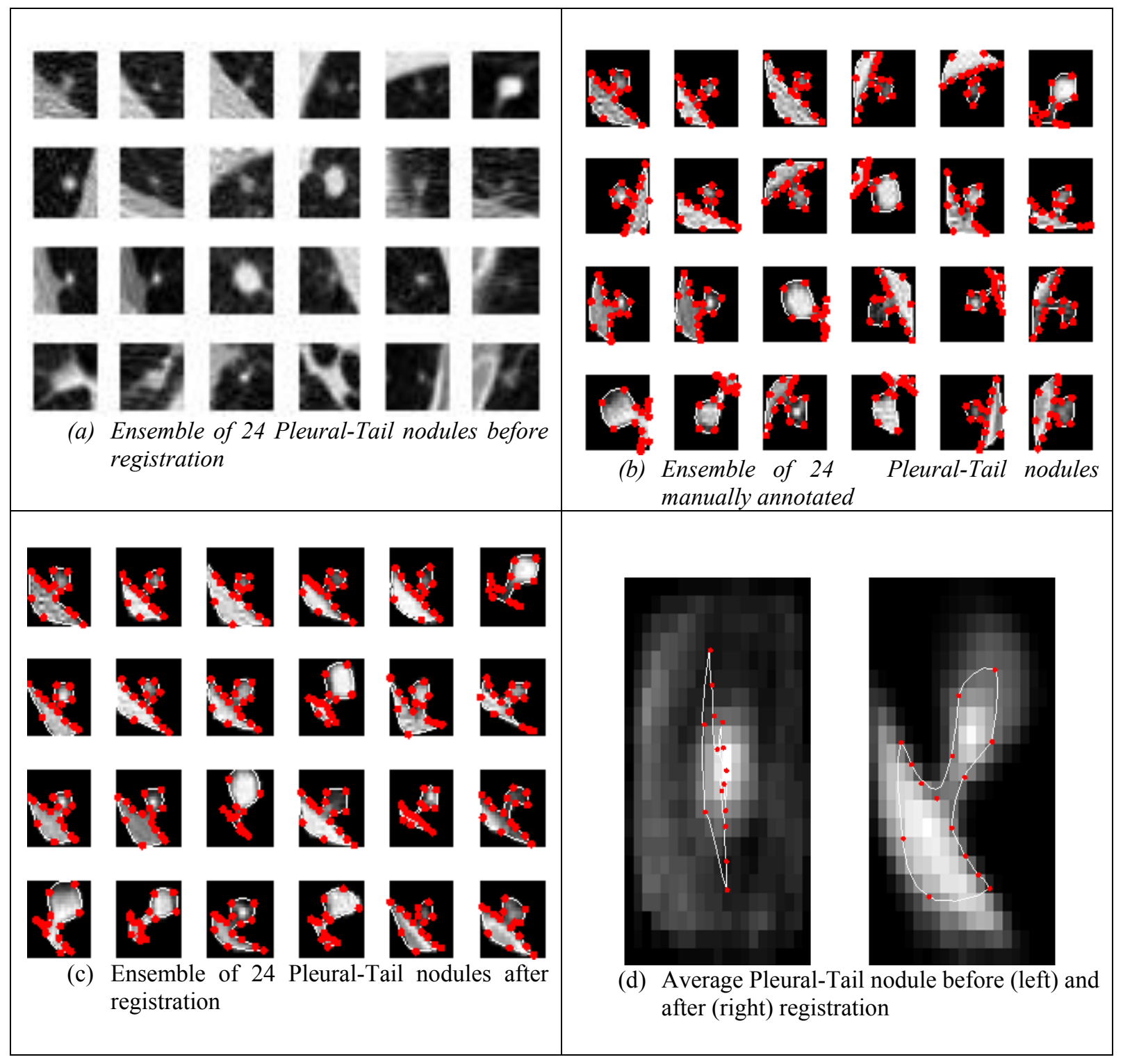

Figure 54: Generating the Pleural Tail nodule. (a) Original (non registered nodules); (b) manually annotated nodules; (c) co-registered (with respect to first nodule) nodules; and (d) average nodule with and without registration. Notice the averaging process creates a nodule which maintains the main characteristics of Pleural Tail nodules. 
There are other approaches that could be employed to perform the group-wise registration, including shape-based approached (e.g., [75]). As shown in Figures 51-54, the registration process leads to capturing the major features of each nodule type, thus the template is more descriptive of the particular nodule. This proves to be the key reason for the enhancements of the sensitivity and specificity of the nodule detection process using template matching as will be shown later on in this chapter.

\section{Template Matching}

As we shown in Chapter 4 using parametric templates, we will start with studying the behavior of the Normalized Cross-Correlation (NCC) for the new templates. For each nodule model, the NCC is obtained over all slices in the ELCAP study with known ground truth. The histogram for the average NCC for all nodule types is shown in Figure 55. The NCC behavior with the new nodule models takes the same general shape as with the parametric nodules (e.g., Figure 40 in Chapter 4), except the distribution function decays a lot faster as we approach a value of 0.5 - thus setting a threshold of 0.5 (to be able to compare with previous results) would result in detecting fewer nodules, but with better sensitivity and specificity as we will illustrate next.

Figure 56-59 illustrates the effectiveness of individual templates in detecting the other nodule types. The idea is that templates of a particular type should latch/match with their own type. Compared to Figure 39 (Chapter 4), we can easily conclude that the new templates indeed provide better sensitivity. This will be further illustrated as we perform the nodule detection using a fusion of the output of the four template models. 


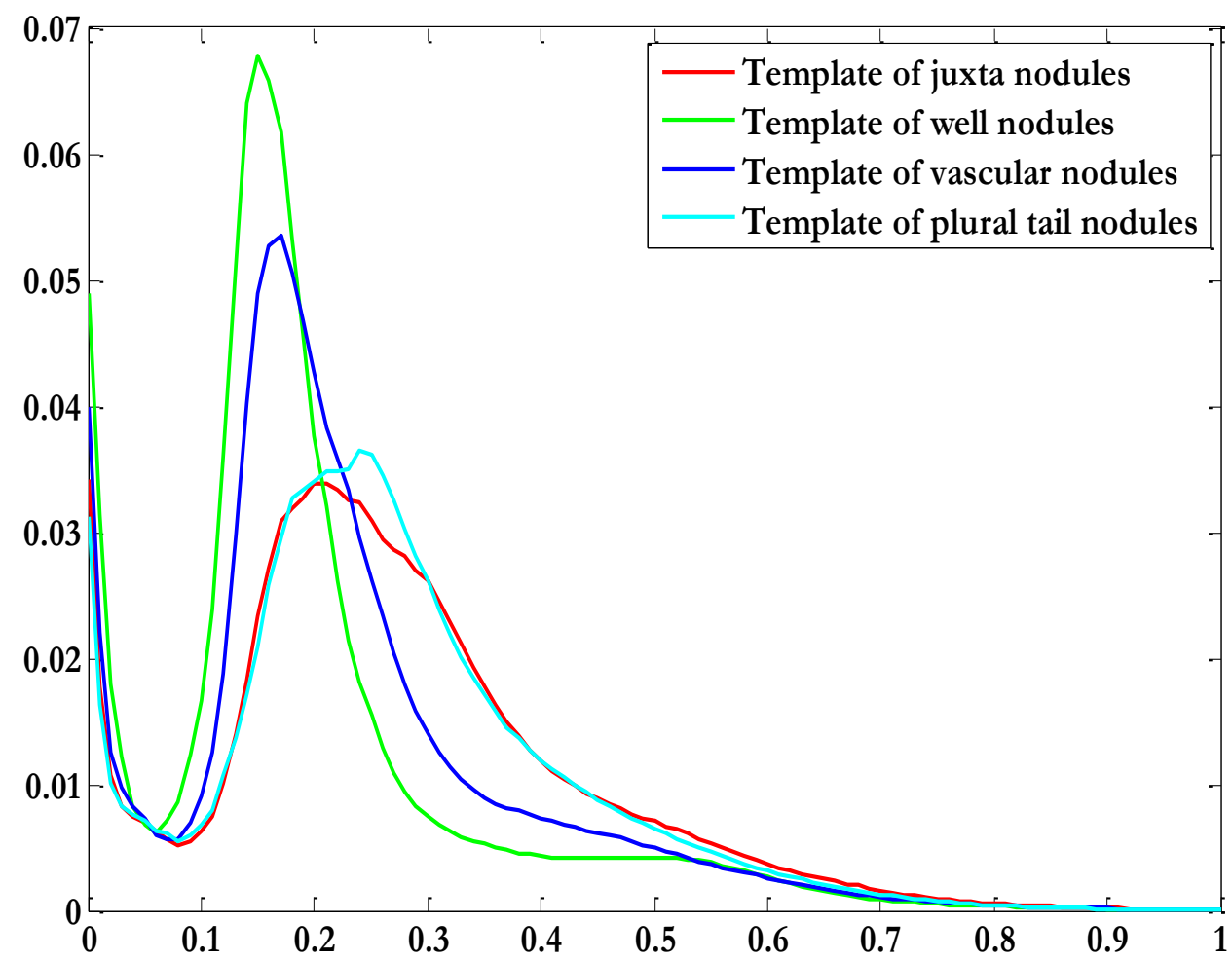

Figure 55: Distribution of the Normalized Cross-Correlation (NCC) for non-parametric templates. Higher NCC values results in less FPs while smaller values provide more FPs. 


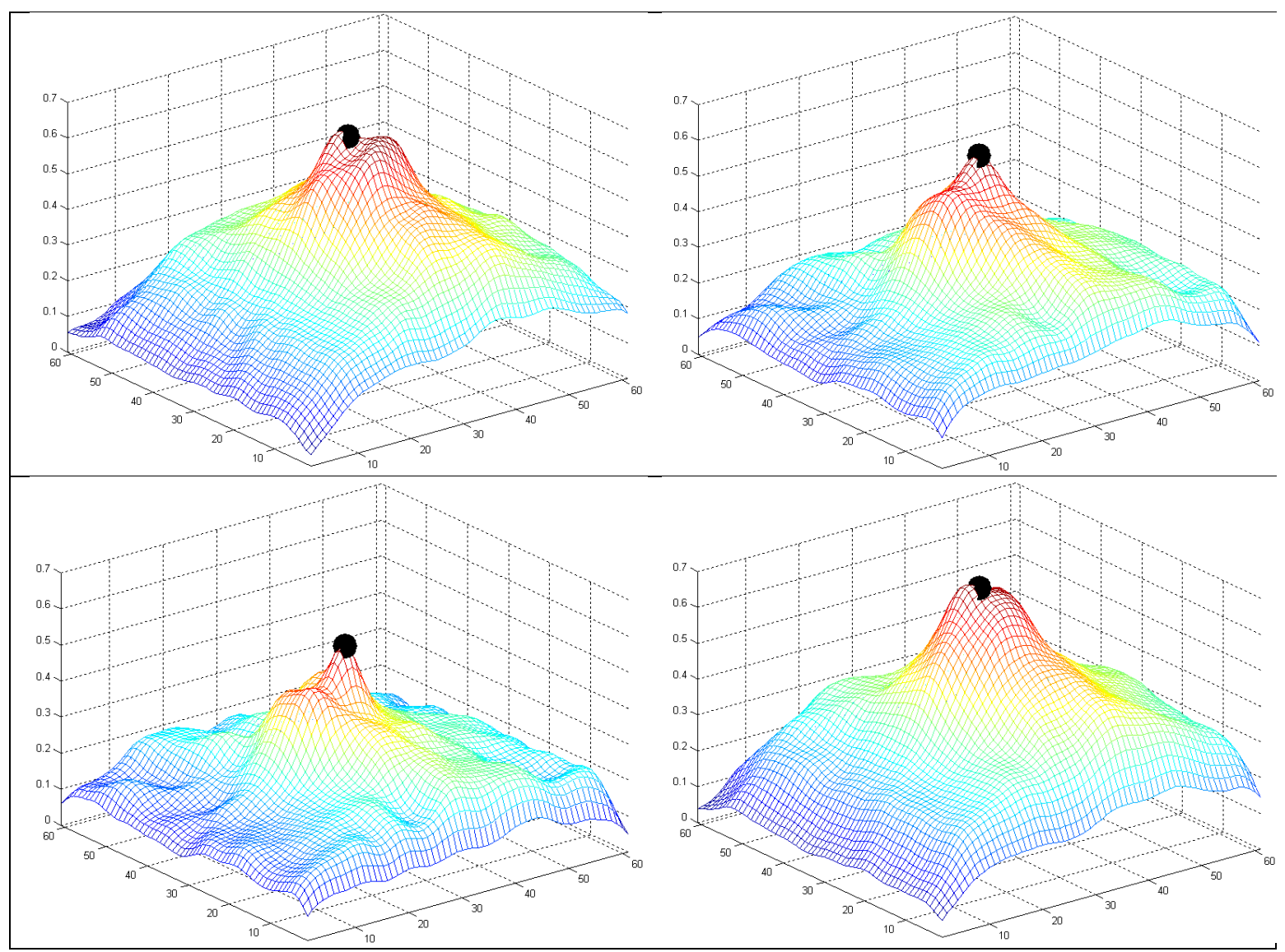

Figure 56: Normalized Cross-Correlation (NCC) for circular templates on regions around the correct nodule location. The figure is the average NCC for the pleural tail nodule type. Upper left is the average NCC for Pleural-Tail nodule using pleural tail template, Upper right is the results for Pleural-Tail nodule using Vascular template, lower left is the results for Pleural-Tail nodule using Well-Circumscribed template, and Lower right shows the results for Pleural-Tail nodule using Juxta-Pleural template. The location of the nodules (as provided by the ELCAP experts), shown in black, may not correspond to the center or centroid of the real nodules. 


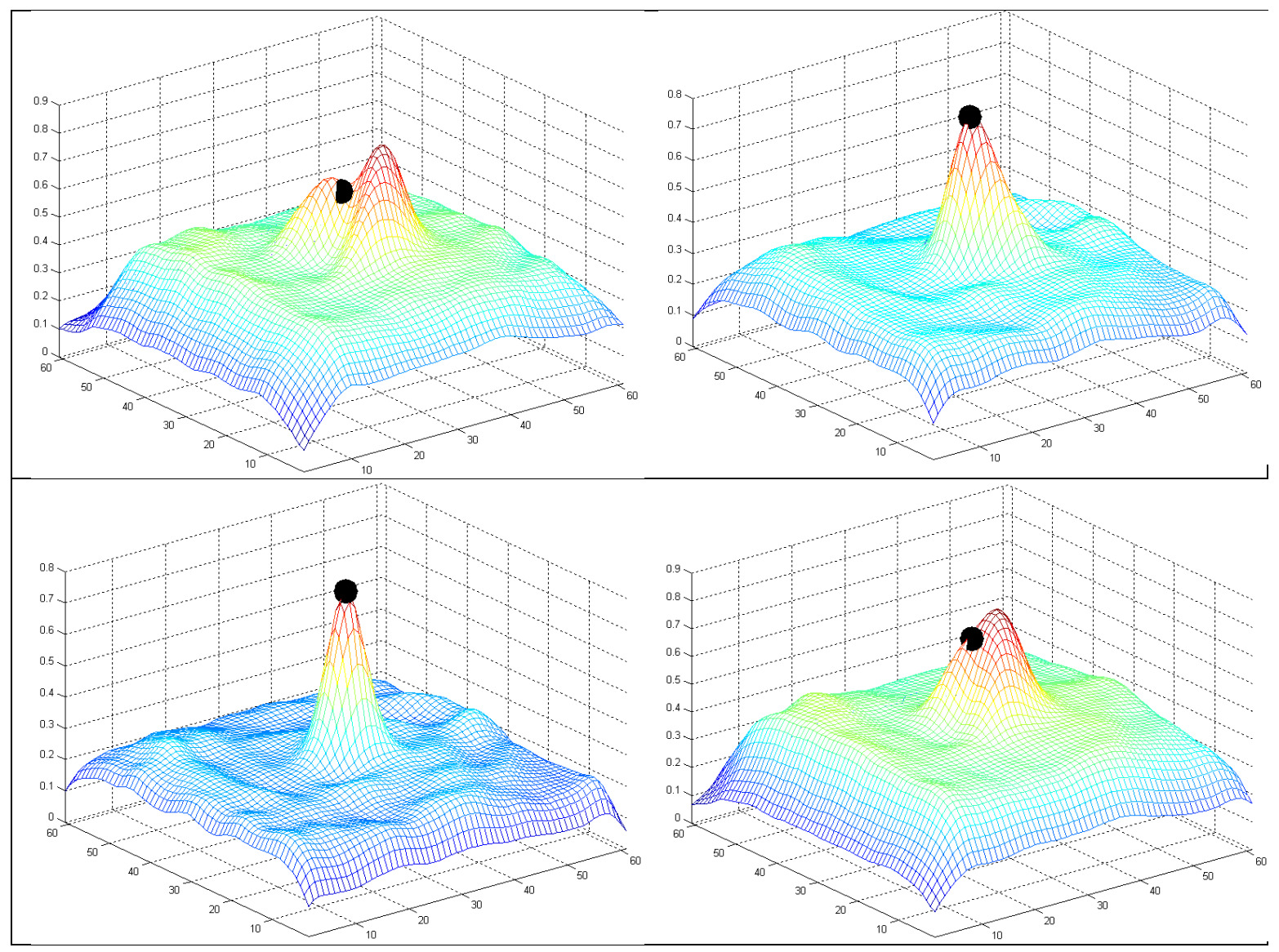

Figure 57: Normalized Cross-Correlation (NCC) for circular templates on regions around the correct nodule location. The figure is the average NCC for the Vascular nodule type. Upper left is the average NCC for Vascular nodule using pleural tail template, Upper right is the results for Vascular nodule using Vascular template, lower left is the results for Vascular nodule using Well-Circumscribed template, and Lower right shows the results for Vascular nodule using Juxta-Pleural template. The location of the nodules (as provided by the ELCAP experts), shown in black, may not correspond to the center or centroid of the real nodules. 


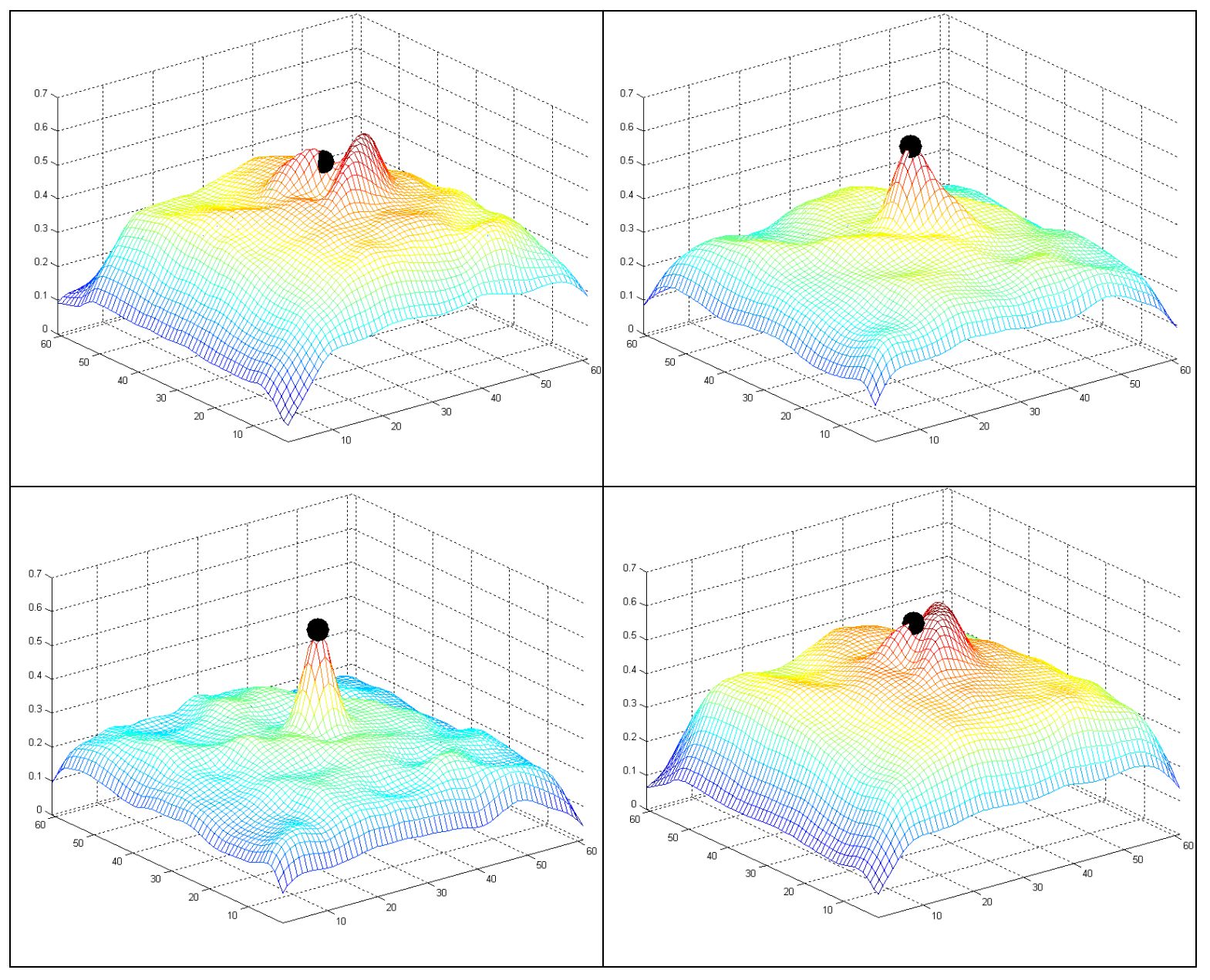

Figure 58: Normalized Cross-Correlation (NCC) for circular templates on regions around the correct nodule location. The figure is the average NCC for the WellCircumscribed type. Upper left is the average NCC for Well-Circumscribed nodule using pleural tail template, Upper right is the results for Well-Circumscribed nodule using Vascular template, lower left is the results for Well-Circumscribed nodule using WellCircumscribed template, and Lower right shows the results for Well-Circumscribed nodule using Juxta-Pleural template. The location of the nodules (as provided by the ELCAP experts), shown in black, may not correspond to the center or centroid of the real nodules. 


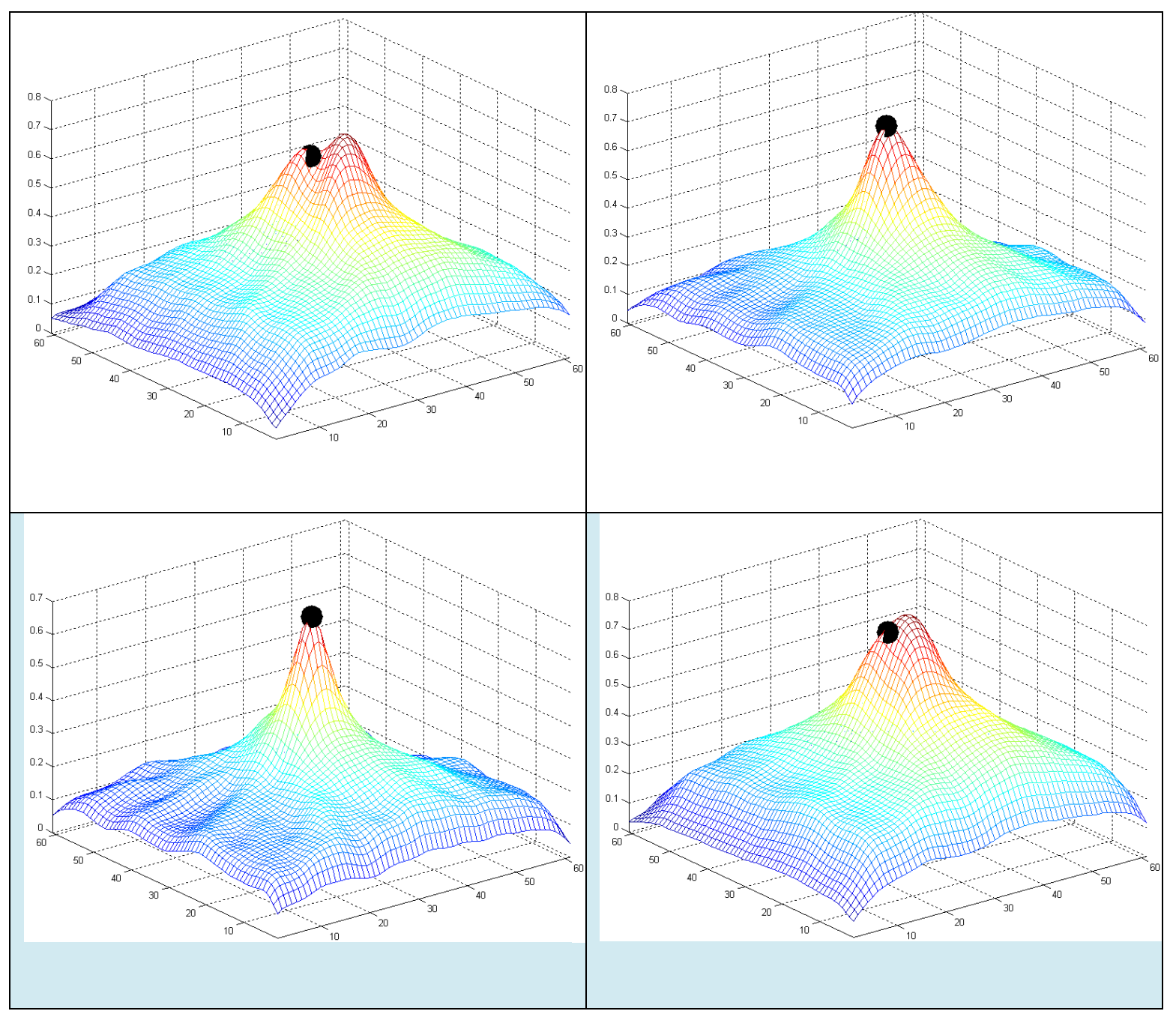

Figure 59: Normalized Cross-Correlation (NCC) for circular templates on regions around the correct nodule location. The figure is the average NCC for the Juxta type. Upper left is the average NCC for Juxta nodule using pleural tail template, Upper right is the results for Juxta nodule using Vascular template, lower left is the results for Juxta nodule using Well-Circumscribed template, and Lower right shows the results for Juxta nodule using Juxta-Pleural template. The location of the nodules (as provided by the ELCAP experts), shown in black, may not correspond to the center or centroid of the real nodules. 


\section{Nodule Detection Using the New Template Models}

The new template models were used for detection of nodules on the ELCAP data. The template matching was performed as before and we used an NCC threshold of 0.5 to be able to compare with the parametric templates discussed in Chapter 4 in terms of sensitivity and specificity. We chose to conduct a decision fusion approach where we use the four templates in the detection process in a serial fashion and the final decision is XOR of the four binary outputs. The output of the template matching from each nodule model is a binary image ( $\mathrm{NCC}$ values rank from zero to 1 ; after thresholding the zeros are NCC values below 0.5 and the ones are otherwise ). In other words, this output image is a black and white representation of where the candidate nodules for the corresponding nodule type are located; binarization is performed onto these images to give any pixels that are black a value of 0 and any white pixels a value of 1 . The four images are Exclusively-ORed (XOR) together to receive a final black and white image that represents the candidate nodule locations for all of the nodule types. The XOR in mathematics and logic is a logical operator that results in a value of true if and only if one or the other but not both results are true. In our case, when we XOR the four images after template matching and binarization only the locations where one of the templates has a nodule detected for that pixel value will be taken as a true candidate nodule. Figure 49 below illustrates this process. 


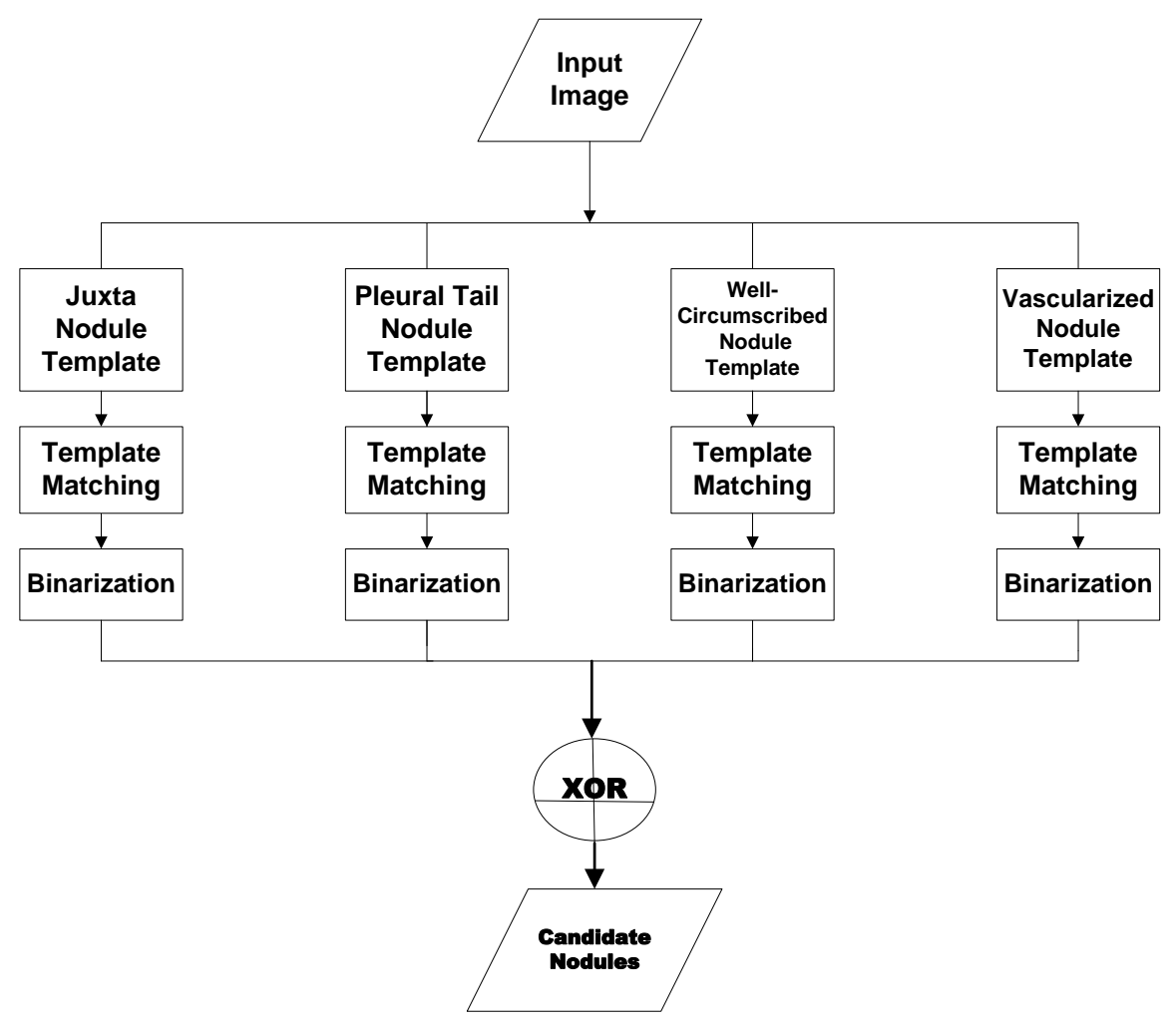

Figure 60: Block diagram of novel nodule detection design

\section{$\underline{\text { E. Experimental Results }}$}

Table 5: Results of template matching (single sweep) using the new template design approach.

\begin{tabular}{|l|l|l|l|l|l|}
\hline \multicolumn{5}{|c|}{ Template generated from training nodules using the new templates } \\
\hline & $\begin{array}{l}\text { All nodule } \\
\text { types }\end{array}$ & $\begin{array}{l}\text { Juxta- } \\
\text { Pleural }\end{array}$ & Well & Pleural-Tail & Vascular \\
\hline Sensitivity & $85.1 \%$ & $94.34 \%$ & $70 \%$ & $94.59 \%$ & $81.25 \%$ \\
\hline Specificity & $86.3 \%$ & $86.52 \%$ & $87.08 \%$ & $83.77 \%$ & $86.65 \%$ \\
\hline
\end{tabular}


Table 6: Results of template matching (single sweep) using the new template design approach for four orientations per nodule type, using step size 45 (total of 16 orientations).

\begin{tabular}{l} 
Template generated from training nodules (using templates with different orientations) \\
using the new templates \\
\hline
\end{tabular}

Compared to Table 3 and 4, we note the improvements in the results of the template matching approach using the new template design. We intend to investigate this approach further using more ensemble sizes and various clinical data sets. We believe that this result is most significant in current efforts in establishing a computerized approach for automatic detection and classification of lung nodules. In fact, we will extend this approach using ensemble of nodules with known pathological classifications.

\section{F. Summary}

This chapter introduced a new approach to model the nodules and generate an intelligent deformable template. The approach is based on statistical shape models on ensemble of nodules. This approach has lead to improvements in the sensitivity and specificity of template matching and lead to significant improvements in false positive reductions.

Nodule modeling work includes [65-68]. The approach described in this chapter is distinct and is more realistic in terms of capturing various characteristics of real nodules as presented in the ensemble. 


\title{
CHAPTER 6
}

\section{CONCLUSIONS AND RECOMMENDATIONS}

\author{
A. Summary \\ This thesis examined computerized analysis of lung nodules in low dose CT (LDCT) \\ scanning. Attempts to create CAD systems based on various imaging modalities have \\ been an active research area for over three decades. With respect to lung cancer, the \\ national and international screening studies aim to test whether CT is better than X-ray \\ for early diagnosis of lung cancer, and whether early detection leads to improved \\ mortality. This latter issue is the most crucial and eventually may lead to making regular \\ LDCT scanning a common practice. From an image analysis prospective, basic CAD \\ system consists of four steps: 1) data smoothing/conditioning; 2) data segmentation to \\ isolate the lung tissues from the rest of the organs in the LDCT scan; 3) nodule detection; \\ and 4) nodule classification - i.e., assigning pathology for a nodule. This thesis dealt with \\ the first three steps. The lack of availability of a database having classified cases of \\ cancer and non cancer did not permit examining the classification issue. It is certainly the \\ ultimate goal of computerized analysis methods and is the element to be pursued as \\ continuation of this work. \\ Below we highlight the logic we followed in our analysis and the conclusions we \\ obtained
}


1) Noise reduction: with respect to data conditioning, we examined the image quality of LDCT scans with focus on the ELCAP study. This data is corrupted by speckle and salt and pepper noise, and an apparent haziness exists in the data. There are many approaches in the literature to address these types of artifacts. Our contribution was a magnifying glass look at the noise statistics in order to devise a data-driven filtering approach that handles these artifacts. We considered two widely usable optimal filtering methods, the Weiner Filter and the Anisotropic Diffusion Filter. We tested the two approaches on the ELCAP data. We concluded that either approach is adequate for noise reduction and reduction of image haziness. We recommended the Anisotropic Diffusion filter with window widths of $7 \times 7$ or $9 \times 9$. Of course, even though not highlighted in the thesis, filtering adds another element of mathematical interest in the sense that it provides a regularization (transfer of discrete/sampled values into a continuous representation) which enables further analysis.

2) Lung Tissues Segmentation: the goal to isolate the lungs tissues from the chest and thoracic regions. We studied a few of the CVIP Lab algorithms that have been developed over the years which has general applicability, including lung segmentation from LDCT scans. Efforts were directed towards two wellestablished and highly tested approaches, one is statistical-based and the other is variational-based. 
- The statistical approach uses the traditional composite random field frame work which considers the image information to contain the details of each of class, and the segmentation process (desired labeling) maps these details into a finite set of labels (classes) exploiting the structure/spatial interaction between neighboring pixels/voxels. This interplay between the low-level process (the intensity information/Hounsfield units in the LDCT) and the high-level process (the labeling/segmentation) provides what is known as the maximum a posteriori (MAP) estimation of the labeling of the information in the image. The MAP estimate may be calculated by various approaches including dynamic programming, simulated annealing, and graph-cuts. All these methods have been used at the CVIP Lab. The statistical method we used (Ali-Farag, 2008) uses Graph Cuts to obtain the MAP solution.

- The variational/level-sets approach generates an implicit curve/surface representation, such that the boundaries between objects in an image may be estimated from the characteristics of the evolution of the curve/surface. This process is governed by a partial differential equation, which uses the intrinsic characteristics in the image (e.g., edge/boundary, shape, and homogeneity information) to devise the solution. This approach is very powerful and handles various situations with occluded and inhomogeneous objects; it is hard to implement and may require manual interventions.

- We examined the LDCT data of the ELCAP study and found its gray level histogram to be distinctly bi-modal. Hence, we devised a thresholding approach 
based on the optimal boundary between the marginal densities in the histogram. Refinements using basic morphological operations was then applied to enhance the boundaries of the lung region. This general approach was implemented by other researchers as well. It is simple and requires minimal manual interventions. Our contribution is the automation of the algorithm, where we model the histogram as a joint probability density function between lungs and non-lungs tissues using the linear model with Gaussian kernels. We employed the wellknown EM algorithm for estimating the components of the linear model, and also the marginal densities of the lungs and the non-lungs regions. All this is performed from the local information (histogram of the LDCT scan) thus virtually no human intervention is needed.

- To test the quality of our segmentation approach with respect to the sophisticated and much more advanced algorithms at the CVIP Lab, we used an ensemble of CT slices and manually segmented the lung tissues. Then we applied the three segmentation approaches to this ensemble. The quality of the registration was tested using the Mutual Information (MI) and the Square Error Distance (SED). The analysis shown in Chapter 3 suggests that our simplified approach provides a comparable result to the other involved approaches; hence we decided to use our approach in the subsequent analysis.

3) Nodule Detection: After smoothing the LDCT scan to reduce noise and haziness, then segmenting the lung tissues, we studied on the inherent characteristics of the lung nodules with respect to the rest of the lung tissues. Nodule detection is 
performed using template matching, a standard process in image analysis. Our contribution is the design of the templates from the characteristics of clinical data. We used the classifications provided in the ELCAP data to design the set of templates. After template detection, a process of reducing/eliminating false positives is conducted. Intelligent nodule design can eliminate a lot of false positives and thus enhances the performance of the final step of the CAD system. In our publications (Elhabian, Farag, et al., 2008) we studied the sensitivity analysis of the template matching. We reported that circular and 45 degrees semicircular nodules provided best performance for the four nodule types in the ELCAP data. This thesis introduced two new contributions which may potentially be of real significance in CAD design.

- In chapter 4 we analyzed the statistics of lung nodules from a large ensemble of nodules from the ELCAP study. We confirmed what the literature reported that the gray level distribution of the nodules tend to decay exponentially from the centroid of the nodules. From the analysis of the probability density of the radial distances of the nodules, we noted that after a distance of 10 pixels from the centroid, the probability density becomes almost zero. This corresponds to a distance of about $5 \mathrm{~mm}$ on the CT scan; which is the length of interesting nodules to the radiologists. Therefore, we have proved empirically that a template of radius 10 pixels (if symmetric would be a window of size $11 \times 11$ or a circle of radius 10) is enough for template matching. This is very important step towards 
reducing the enormous computing time associated with the template matching process.

- In chapter 5 we took a fresh look at the issue of template design. We employed several of the tools in the computer vision literature to study the statistics and geometry of the nodules in order to design what we called "intelligent" template which enhance the sensitivity and the specificity of the nodule detection process. This has not been reported before in the CT-based nodule detection literature and is of great promise. The gist of this discovery, as outlined in chapter 5 , is that a smart template model may be created from a descriptive ensemble of nodules and used instead of the shot gun methods which employ non realistic template shapes.

\section{$\underline{\text { B. Extensions }}$}

The extensions to this work are very evident and boil to two major fronts:

1) Authenticating the intelligent nodule modeling approach in Chapter 5; and

2) Addressing the last step in the CAD system. At the CVIP Lab there exist clear plans for accomplishing these two goals.

An IRB has just been approved to allow access to the Louisville Lung Screening study. Members of this thesis committee (Drs. LaRocca and Falk) will play crucial rules in providing expertise in manually generating data samples for benign and malignant nodules, and designing the protocol to test the performance of the computerized approach versus human experts. 
Once we obtain access to the Louisville Lung Screening Study the work conducted in this thesis for the first steps of the computer-based analysis of LDCT of the chest will be assessed for this database (see figures 5 or 25 for the system block diagram). Meaning lung segmentation of the lung tissue will be performed (if filtering is necessary, will be conducted in the same manner as in this thesis), then nodule detection using both methods stated in chapters 4 and 5. The results from ELCAP and the Louisville Lung Screening datasets will be analyzed against each other to conclude if better results have been obtained. After completion of the tasks executed in this thesis on the new database research focus will be geared towards reaching the optimal goal of our approach, classification of lung nodules.

There are various paths that can be taken to begin this extremely important endeavor. First, we can continue from the point of nodule detection in 2D and perform 2D false positive and false negative reduction. Reduction of false positives and false negatives can be performed in numerous fashions one method is further classifying each candidate nodule region based on extracted features [74], this can be accomplished by fitting an ellipse which has the same normalized second central moments as the candidate region. Examples of shape descriptors that can be used as classification features are:

1) Area: number of pixels in the candidate nodule region

2) Major axis length: the length in pixels of the major axis of the fitted ellipse.

3) Minor axis length: the length in pixels of the minor axis of the fitted ellipse.

4) Eccentricity: A value between zero and one which is the ratio of the distance between the major axis length and the foci of the fitted ellipse. 
5) Perimeter: distance between each adjoining pair of pixels around the border of the region.

6) Solidity: the proportion of the pixels in the convex hull that are also in the region.

Once knowledge of the nodule descriptors are obtained this information can be used to eliminate the amount of false negatives and positives. This is just one possible method for reduction. The next step would be the final step in the CVIP lab approach, the classification step. Information of benign and malignant, calcified and non-calcified nodules and so on would be needed to understand the physical shape and size and who it various over time. An approach similar to that of chapter 5 could be used to form templates of the various nodule types in the Louisville Lung Screening Study by cropping an ensemble of the nodules, registering them using Procrustis and then perform the template sweeping-method using these "Smart templates" to identify the nodules detected into further categories so a diagnosis can be given by a doctor.

Another direction that can be taken is to perform 3D segmentation and then develop 3D templates to be used for 3D template matching which would also execute the step of false positive reduction simultaneously, since a volume of slices is being used thus making the nodules and anatomy 3D in shape so depth perception of the nodules can be measured and used in the template designs. This would lead right into 3D nodule classification into the various categories.

Finally, many modern computer vision approaches in shape analysis will be find direct applicability to this research. In particular, we plan to explore shape analysis 
methods (e.g., Fourier Descriptors, Curvatures, Statistical Shape measures, etc.) to further explore the inherent characteristics of nodules. Likewise, vis-a-vis detection we plan to explore the concept of matched filtering using the new template models; this should provide physical meaning to what is been detected and considered a nodule. Finally, multi-scale approaches (e.g., scale space methods) may prove useful in the classification of candidate nodules into pathologies. These extensions are among the ideas that will be explored on various clinical studies of chest imaging as they become available. 


\section{REFERENCES}

[1] Lung Cancer webpage http://www.cancer.gov/cancertopics/types/lung

[2] National Institute of Health www.nih.gov

[3] Jewish Hospital www.jewishhospital.org/heartlunginst.html

[4] Renato La Rocca, Monte Martin, Robert Falk, Laura Brenzel and Margaret Oechsli, "Results of a Randomized Prospective Pilot Screening Trial of Annual Low-Dose Spiral Computed Tomography (LDCT) versus Annual Chest Radiography (x-ray) in patients at High Risk for Lung Cancer: Louisville Community Lung Cancer Screening Study,” 2009 (Submitted manuscript).

[5] ELCAP public lung image database, http://www.via.cornell.edu/databases/lungdb.html

[6] C. T. Henschke, et al. "Early lung cancer Action Project: Overall Design and Findings from baseline Screening," Cancer.2000 (Suppl), Vol.89 (11), pp. 2474$2482,2000$.

[7] S. J. Swenson, et al., Lung cancer screening with CT: Mayo Clinic experience, Radiology, Vol. 226, pp. 756-761, 2003.

[8] R. P. Petersen and D. H. Harpole "Computed Tomography Screening for the Early Detection of Lung Cancer," The Journal of the National Comprehensive Cancer Network, Vol. 4 pp.1-4, 2006.

[9] C. I. Henschke, et al. "Survival of patients with stage I lung cancer detected on CT screening," New England Journal of Medicine, Vol. 355, pp. 1763-1771, 2006.

[10] W. J. Kostis, et al., "Small pulmonary nodules: reproducibility of threedimensional volumetric measurement and estimation of time to follow-up," Radiology, Vol. 231, pp. 446-52, 2004.

[11] C. A. Van Iersel, et al., "Risk-based selection from the general population in a screening trial: Selection criteria, recruitment and power fro the Dutch-Belgian randomized lung cancer multi-slice CT screening trial (NELSON)," International Journal of Cancer, Vol. 120, pp. 868-874, 2006.

[12] W. J. Kostis, A.P. Reeves, D. F. Yankelevitz, and C. I. Henschke, "Three dimensional segmentation and growth-rate estimation of small pulmonary nodules 
in helical CT images," Medical Imaging IEEE Transactions Vol. 22, pp. 12591274, 2003.

[13] I. Sluimer, A. Schilham, M. Prokop, and B. van Ginneken, "Computer Analysis of Computed Tomography Scans of the Lung: A Survey," IEEE Transactions on Medical Imaging, vol. 25, No. 4, pp. 385-405, April, 2006.

[14] Shireen Y. Elhabian, Amal A. Farag, Salwa Elshazly and Aly A Farag, "Sensitivity of Template Matching for Pulmonary Nodule Detection: A Case Study," 4th Cairo International Biomedical Engineering Conference (CIBEC 2008), Cairo, Egypt December 18-20, 2008, pp. 110 -114.

[15] Aly A. Farag and Hassan Abdemunim (2004), "Adaptive segmentation of multimodal 3D data using robust level set techniques," in Proc. International Conference on Medical Image Computing and Computer-Assisted Intervention (MICCAI' 04), Saint Malo, France, September 26-29, 2004, pp. 143-150.

[16] Asem Ali and Aly A. Farag, "Automatic Lung Segmentation of Volumetric LowDose CT Scans Using Graph Cuts," 4th International Symposium on Visual Computing (ISVC-08), Las Vegas, December 1-3, 2008, pp. 258-267.

[17] S. G. Armato, M. L. Giger, C. J. Moran, J. T. Blackburn, K. Doi and H. MacMahon, "Computerized detection of pulmonary nodules on CT scans.," Radiographics Vol. 19, pp. 1303-1311, 1999.

[18] A. A. Farag, A. El-Baz, G.Gimelfarb, R. Falk and S. G. Hushek, “Automatic detection and recognition of lung abnormalities in helical CT images using deformable templates," Proc. of International Conference on Medical Image Computing and Computer-Assisted Intervention, MICCAI-2004, September 2629, 2004, pp. 856-864.

[19] A. A. Farag, A. El-Baz, and G. Gimel'farb, “Quantitative Nodule Detection in Low Dose Chest CT Scans: New Template Modeling and Evaluation for CAD System Design," Proc. of International Conference on Medical Image Computing and Computer-Assisted Intervention (MICCAI-05), Palm Springs, California, USA, October 26-29, 2005, pp. 720-728.

[20] A. Farag, A. El-Baz, G. L. Gimel'farb, R. Falk, M. Abou El-Ghar, T. Eldiasty, S. Elshazly, "Appearance Models for Robust Segmentation of Pulmonary Nodules in 3D LDCT Chest Images," Proc. of International Conference on Medical Image Computing and Computer-Assisted Intervention (MICCAI'06), Copenhagen, Denmark, October 1-6, 2006, pp. 662-670. 
[21] A. Rosenfeld and A. Kak, Digital Picture Processing, Vol. 1, 2nd edition, Academic Press, NY, 1982.

[22] J. Parker, Image Reconstruction in Radiology, CRC Press, Boca Raton, Florida, 1990.

[23] J. Prince and J. Links, Medical Imaging Signals and Systems, Prentice-Hall, New Jersey, 2006.

[24] A. A. Farag, Statistical Models in Biomedical Image Analysis, Technical Report, CVIP Lab, University of Louisville, 2000.

[25] A. A. Farag and E. J. Delp, "Image Segmentation Based on Composite Random Field Models," Journal of Optical Engineering, Vol. 12, pp. 25942607, December 1992.

[26] R. C. Dubes and A. K. Jain, "Random Field Models in Image Analysis," Journal of Applied Statistics, Vol. 16, pp. 131-164, 1989.

[27] R. Kindermann and J. Snell, Markov Random Fields and Their Applications, American Mathematical Society, Providence, Rhode Island, 2000.

[28] J. Lim, Two-Dimensional Signals and Image Processing, Prentice-Hall, New Jersey, 1990.

[29] P. Perona and J. Malik, "Scale-Space and Edge Detection Using Anisotropic Diffusion," IEEE Transactions on Pattern Analysis and Machine Intelligence, PAMI, Vol. 12, No. 7, pp. 629-639, July 1990.

[30] G. Grieg, O. Kubler, R. Kikinis, and F. A. Jolesz, "Nonlinear Anisotropic Filtering of MRI Data," IEEE Transactions on Medical Imaging, Vol. 11, No. 2, pp. 221-232, June 1992.

[31] A. P. Dempster, N. M. Laird, and D. B. Rubin, "Maximum likelihood from incomplete data via the EM algorithm," J. Roy. Stat. Soc. B, vol. 39, pp. 1-38, 1977.

[32] R. Duda, P. Hart and D. Stork, Pattern classification 2nd, Wiley, New York, 2000.

[33] C. J. McLachlan, The EM Algorithm and Extensions, Wiley, New York, 1997.

[34] R. Redner and H. Walker, "Mixture densities, maximum likelihood and the EM algorithm (review)," SIAM Rev., Vol. 26, pp. 195-237, 1984. 
[35] Asem Ali and Aly A. Farag, "Density Estimation using a new AIC-type Criterion and the EM algorithm for a Linear Combination of Gaussians," Proc. of IEEE International Conference on Image Processing (ICIP'08), San Diego, California, pp. 3024-3027, October 12-15, 2008.

[36] Aly A. Farag, Ayman El-Baz, Georgy L. Gimel'farb, "Precise segmentation of multimodal images," IEEE Transactions on Image Processing Vol. 15, no. 4, April 2006, pp. 952-968.

[37] S. Hu, E. A. Hoffman and J. M. Reinhardt, "Automatic lung segmentation for accurate quantitation of volumetric X-ray CT images," IEEE Transactions on Medical Imaging, Vol. 20, pp. 490-498, 2001.

[38] M. S. Brown, M. F. McNitt-Gray, N. J. Mankovich, J.Goldin, J.Hiller, L. S. Wilson and D. R. Aberle, "Method for segmenting chest ct image data using an anatomical model: Preliminary results," IEEE Transactions on Medical Imaging Vol. 16, 828-839, 1997.

[39] I. Sluimer, M. Prokop, B. van Ginneken, "Toward automated segmentation of the pathological lung in CT," IEEE Transactions on Medical Imaging, Vol. 24, 10251038, 2005.

[40] L. Zhang, E. A. Hoffman and J. M. Reinhardt, "Atlas-driven lung lobe segmentation in volumetric x-ray ct images," Proc. of the SPIE, vol. 5031, pp. 306-315, 2003.

[41] Y. Boykov, and G. Funka-Lea, "Graph cuts and efficient N-D image segmentation,” International Journal of Computer Vision 70, 109-131, 2006.

[42] Y. Boykov and M. P. Jolly, "Interactive organ segmentation using graph cuts," Proc. of Medical Imaging Computing and Computer-Assisted Interventions, MICCAI 2000, Pittsburgh, Pennsylvania, October 11-14, 2000, pp. 276-286.

[43] H. Lombaert, Y. Sun, L.Grady and C. Xu, "A multilevel banded graph cuts method for fast image segmentation," Proc. IEEE International Conference on Computer Vision, ICCV-05, Vol. I, pp. 259-265, 2005.

[44] S. Chen, L. Cao, J. Liu and X.Tang, "Automatic segmentation of lung fields from radiographic images of sars patients using a new graph cuts algorithm," Proc. International Conference on Pattern Recognition, ICPR-06, Vol. 1, pp. 271-274, 2006.

[45] M. Kass, A. Witkin and D. Terzopoulos, "Snakes: active contour models," International Journal of Computer Vision, Vol. 1, pp. 321-331, 1987. 
[46] S. Osher and J. Sethian, "Fronts propagating with curvature-dependent speed: algorithms based on Hamilton-Jacobi formulations," J. Comp. Phys., Vol. 79, pp. 12-49, 1988.

[47] J. A. Sethian, Level set methods and fast marching methods, Cambridge University Press, Cambridge, UK, 1999.

[48] T. Chan and L. Vese, "Active contours without edges," IEEE Transactions on Image Processing., Vol. 10, pp. 266-277, 2001.

[49] H. Abdelmunim and A. A. Farag, "A Shape-based Segmentation Approach: An Improved Technique using Level Sets," Proc. of International Conference on Computer Vision (ICCV), Beijing, China, October 15-21, 2005, pp. 930-935.

[50] Hossam Abd EL Munim and Aly A. Farag, "Curve/Surface Representation and Evolution using Vector Level Sets with Application to the Shape-based Segmentation Problem," IEEE Transactions on Pattern Analysis and Machine Intelligence - Vol. 29, No. 6, pp. 945-958, June 2007.

[51] G. Kloecker, et al., "Lung Cancer in the US and in Kentucky," Proc. Kentucky Medical Association (KMA), pp.159-164, 2007.

[52] C. F. Mountain, "Revisions in the international system for staging lung cancer," Chest Vol.111 pp.1710-1717, 1997.

[53] A. Gajra, et al, "Impact of tumor size on survival in stage IA non-small cell lung cancer: a case for subdividing stage IA disease," Lung Cancer Vol. 42 pp.51—57, 2003.

[54] B. Zaho, G. Gamsu, M. S. Ginsberg, L. Jiang and L. H. Schwartz, "Automatic Detection of small lung nodules on CT utilizing a local density maximum algorithm.," Journal of Applied Clinical Medical Physics , Vol. 4, 2003.

[55] S. G. Armato, M. L. Giger, C. J. Moran, J. T. Blackburn, K. Doi and H. MacMahon, "Computerized detection of pulmonary nodules on CT scans.," Radio Graphics Vol. 19, pp. 1303-1311, 1999.

[56] M. Yang, S. Periaswamy and Y. Wu, "False Positive Reduction In Lung Ggo Nodule Detection With 3D Volume Shape Descriptor," Proceedings IEEE Int'1 Conference on Acoustics, Speech and Signal Processing, Hawaii, 2007.

[57] P. M. Boiselle and C. S. White, New techniques in thoracic imaging, Dekker, New York, 2002 
[58] H. Satoh, Y. Ukai, N. Niki, K. Eguchi, K. Mori, H. Ohmatsu, R. Kakinuma, M. Kaneko and N. Moriyama, "Computer aided diagnosis system for lung cancer based or retrospective helical CT images," Proceedings SPIE pp.1324-1335, 1999.

[59] Y. Lee, T. Hara, H. Fujita, S. Itoh and T. Ishigaki, "Automated detection of pulmonary nodules in helical CT images based on an improved template matching technique,” IEEE Transactions on Medical Imaging, Vol. 20 pp. 595-604, 2001.

[60] S. Y. Elhabian, H. Abdelmunim, S. Elshazly, A. Farag, M. Aboelghar, "Experiments on Sensitivity of Template Matching for Lung Nodule Detection in Lowdose CT Scans,” ISSPIT, pp. 1040-1046, 2007.

[61] Lindsey Smith, A Tutorial on Principle Component Analysis, "http://www.cs.otago.ac.nz/cosc453/student_tutorials/principal_components.pdf

[62] Spherical harmonics - http://mathworld.wolfram.com/SphericalHarmonic.html

[63] M. B. Stegmann and David Delgado Gomez. A Brief Introduction to Statistical Shape Analysis, Technical University of Denmark, Lyngby, 2002.

[64] M. J. Cairns. An Investigation into the use of 3D Computer Graphics for Forensic Facial Reconstruction, Glasgow University, 2000.

[65] K. Murphy, B. van Ginneken, J.P.W. Pluim, S. Klein, and M. Staring. "Semiautomatic Reference Standard Construction for Quantitative Evaluation of Lung CT Registration.” In Proc. of International Conference on Medical Image Computing and Computer-Assisted Intervention 2008, In Press.

[66] A. Pousse, L. Pastor, P. Manzoni, M. Parmentier, B. Kastler. "Pulmonary Nodule Distribution Modeling as a Diagnostic Tool for HRCT Image Analysis.” IEEE Transactions on Nuclear Science 51, 2004.

[67] K. Murphy, A. Schilham, H. Gietema, M. Prokop, B. van Ginneken. “Automated Detection of Pulmonary Nodules from Low-Dose Computed Tomography Scans using a Two-Stage Classification System based on Local Image Features." Medical Imaging 2007.

[68] M. Stegmann, "Active Appearance Models: Theory, Extension and Cases," MS Thesis, IMM DTU, 2000

[69] T. F. Cootes and C. J. Taylor, "Statistical Models of Appearance for Computer Vision," Technical Report, ISBE, University of Manchester, 2004 
[70] Matlab, www.mathworks.com

[71] Amal A. Farag, Shireen Elhabian, Aly A. Farag, Dongqing Chen, Robert Falk, and Renato LaRocca, "Quantification of Nodule Detection in Chest CT: A Clinical Investigation of Early Detection of Lung Cancer Using LDCT Scans," International Conference on Medical Imaging Computing and Computer Assisted Interventions ,MICCAI-09, London, UK (submitted).

[72] Amal A. Farag, Aly A. Farag, Shireen Y. Elhabian, Asem M. Ali, "Parametric Density Estimation Using Generalized Linear Model," International Conference on Image Processing, ICIP-09, Cairo, November 2009 (submitted).

[73] Amal A. Farag, et al. "A new Template Model for Automatic Detection of Lung Nodules from LDCT scans," Academic Journal of Radiology (In preparation).

[74] Yang, M., Periaswamy, S., Wu, Y.: False Positive Reduction In Lung Ggo Nodule Detection With 3D Volume Shape Descriptor. In: Proceedings IEEE Int'1 Conference on Acoustics, Speech and Signal Processing, Hawaii (2007).

[75] H. Abdelmunim and A. A. Farag, "Shape Representation and Registration using Vector Distance Functions," Proc. of IEEE Conference on Computer Vision and Pattern Recognition (CVPR'07), Minneapolis, MN, June 18-23, 2007, pp. Y1-Y8. 
Amal A. Farag was born in West Lafayette, Indiana, USA, moved to Louisville, Kentucky in 1990, where she received all her education. She received the High School Diploma from Louisville Traditional Male High School in 2003. She joined the University of Louisville in 2003, where she received the Bachelor degree in Electrical and Computer Engineering in May 2008. She is scheduled to receive her Masters of Engineering Degree in May 2009. She is interested in biomedical research and may pursue an academic career. Since 2007 she has been a graduate research assistant at the Computer Vision and Image Processing Laboratory, University of Louisville, USA, working on biomedical image analysis with focus on lung cancer. Among her outside interests are multiethnic culture and music. She has been a member of student organizations at the University of Louisville and has traveled all over Europe and a frequent traveler to Egypt. She is particularly fond of Arabic music and movies. 


\section{Amal A. Farag}

Objective: Interested in research and may pursue an academic career. Currently, graduate research assistant at the Computer Vision and Image Processing Laboratory, University of Louisville, USA, working on biomedical image analysis with focus on lung cancer.

\section{Education}

\begin{tabular}{|l|l|l|l|}
\hline Degree & Year & Place & Area \\
\hline $\begin{array}{l}\text { Bachelor of } \\
\text { Science }\end{array}$ & May 2008 & $\begin{array}{l}\text { University of } \\
\text { Louisville }\end{array}$ & $\begin{array}{l}\text { Electrical and } \\
\text { Computer Engineering }\end{array}$ \\
\hline Engineering & May 2009 & $\begin{array}{l}\text { University of } \\
\text { Louisville }\end{array}$ & $\begin{array}{l}\text { Electrical and } \\
\text { Computer Engineering }\end{array}$ \\
\hline Doctoral Student & May 2009 & $\begin{array}{l}\text { University of } \\
\text { Louisville }\end{array}$ & $\begin{array}{l}\text { Electrical and } \\
\text { Computer Engineering }\end{array}$ \\
\hline
\end{tabular}

Master of Engineering Thesis: Lung Cancer: Nodule detection and False Positive Reduction from LDCT Scans. Advisors: Dr. James Graham and Dr. Aly A. Farag, May 2009.

\section{Employments}

\begin{tabular}{|l|l|}
\hline \multicolumn{1}{|c|}{ Date } & \multicolumn{1}{c|}{ Place } \\
\hline $\begin{array}{l}2007- \\
\text { Current }\end{array}$ & $\begin{array}{l}\text { Research Assistant, Computer Vision and Image Processing } \\
\text { Laboratory, University of Louisville, USA }\end{array}$ \\
\hline 2006 & $\begin{array}{l}\text { Three Semester Engineering Co-OP, Computer Vision and } \\
\text { Image Processing Laboratory, University of Louisville, USA }\end{array}$ \\
\hline
\end{tabular}

$\underline{\text { Honors }}$

2009 Master of Engineering with Highest Honors, University of 
Louisville; Graduate GPA 4.0/4.0

2009

$2^{\text {nd }}$ place, overall achievements in the Engineering Day

Competition, University of Louisville, March 2009

2008

$1^{\text {st }}$ place winner of Robotics Design Competition, University of

Louisville

Course Work: Typical ABET accredited Electrical and Computer Engineering curricula with research focus on signals and systems, probability theory, computer vision, biomedical imaging and computing. Software tools include MatLab, $\mathrm{C} / \mathrm{C}++$ and optimization techniques.

\section{Publications}

1. Amal A Farag, Shireen Elhabian, Abdelrehim Ahmed and Aly A Farag, "Noise Analysis of SFS Algorithm Formulated Under Various Imaging Conditions," 4rd International Symposium on Visual Computing (ISVC-08), Las Vegas, Nevada, USA, pp. 803-812, December 1-3, 2008.

2. Shireen Y. Elhabian, Amal A. Farag, Salwa Elshazly and Aly A Farag, Sensitivity of Template Matching for Pulmonary Nodule Detection: A Case Study, 4th Biomedical Engineering Conference, CIBEC 2008, Cairo 1820 Dec. 2008 Page(s):110 -114.

\section{Submitted Articles}

3. Amal A. Farag, Shireen Elhabian, Aly A. Farag, Dongqing Chen, Robert Falk, and Renato LaRocca, "Quantification of Nodule Detection in Chest CT: A Clinical Investigation of Early Detection of Lung Cancer Using LDCT Scans," International Conference on Medical Imaging Computing and Computer Assisted Interventions ,MICCAI-09, London, UK

4. Amal A. Farag, Aly A. Farag, Shireen Y. Elhabian, Asem M. Ali, "Parametric Density Estimation Using Generalzied Linear Model," 
International Conference on Image Processing, ICIP-09, Cairo, November 2009.

\section{PROFESSIONAL REFERENCES}

1. Dr. James Graham, Professor and Chairman, Department of Electrical and Computer Engineering, University of Louisville, USA; E-mail: ihgrah01@lousiville.edu

2. Dr. Aly A. Farag, Professor of Electrical and Computer Engineering, University of Louisville, USA; E-mail: aly.farag@louisville.edu

3. Dr. Mohamed N. Ahmed, Adjunct Professor, Department of Electrical and Computer Engineering, University of Louisville, and Senior Scientist - Lexmark International, Lexington, Kentucky; E-mail: mahamed@lexmark.com 